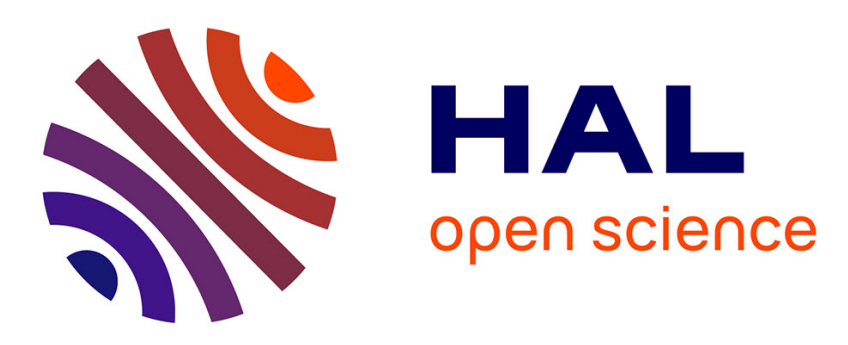

\title{
An overview of recent results on Nitsche's method for contact problems
}

Franz Chouly, Mathieu Fabre, Patrick Hild, Rabii Mlika, Jerome Pousin, Yves

Renard

\section{- To cite this version:}

Franz Chouly, Mathieu Fabre, Patrick Hild, Rabii Mlika, Jerome Pousin, et al.. An overview of recent results on Nitsche's method for contact problems. UCL Workshop 2016, UCL (University College London), Jan 2016, London, United Kingdom. pp.93-141, 10.1007/978-3-319-71431-8_4 . hal-01403003v2

\section{HAL Id: hal-01403003 \\ https://hal.science/hal-01403003v2}

Submitted on 1 Feb 2019

HAL is a multi-disciplinary open access archive for the deposit and dissemination of scientific research documents, whether they are published or not. The documents may come from teaching and research institutions in France or abroad, or from public or private research centers.
L'archive ouverte pluridisciplinaire HAL, est destinée au dépôt et à la diffusion de documents scientifiques de niveau recherche, publiés ou non, émanant des établissements d'enseignement et de recherche français ou étrangers, des laboratoires publics ou privés. 


\title{
An overview of recent results on Nitsche's method for contact problems
}

\author{
Franz Chouly, Mathieu Fabre, Patrick Hild, Rabii Mlika, Jérôme Pousin, and Yves Renard
}

\begin{abstract}
We summarize recent achievements in applying Nitsche's method to some contact and friction problems. We recall the setting of Nitsche's method in the case of unilateral contact with Tresca friction in linear elasticity. Main results of the numerical analysis are detailed: consistency, well-posedness, fully optimal convergence in $H^{1}(\Omega)$-norm, residual-based a posteriori error estimation. Some numerics and some recent extensions to multibody contact, contact in large transformations and contact in elastodynamics are presented as well.
\end{abstract}

Keywords: contact, friction, finite elements, Nitsche's method.

AMS Subject Classification: 65N12, 65N15, 65N30, 74M10, 74M15, 74M20.

\section{Introduction}

For a wide range of systems in structural mechanics, it is crucial to take into account contact and friction between rigid or elastic bodies. Among numerous applications, let us mention foundations in civil engineering, metal forming processes, crash-tests of cars, design of car tires (see, e.g., [90]). Contact and friction conditions are usually formulated with a set of inequalities and non-linear equations on the boundary of each body, with corresponding unknowns that are displacements, velocities and surface stresses. Basically, contact conditions allow to enforce non-penetration on the whole candidate contact surface, and the actual contact surface is not known in advance. A friction law may be taken into account additionally, and various models exist that correspond to different surface properties, the most popular one being Coulomb's friction (see, e.g., [64] and references therein).

Franz Chouly

Laboratoire de Mathématiques de Besançon - UMR CNRS 6623, Université Bourgogne Franche-Comté, 16 route de Gray, 25030

Besançon Cedex, France. e-mail: franz.chouly@univ-fcomte.fr

Mathieu Fabre

EPFL SB MATHICSE (Bt. MA), Station 8, CH 1015 Lausanne, Switzerland. Istituto di Matematica Applicata e Tecnologie, Informatiche "E. Magenes" del CNR, via Ferrata 1, 27100 Pavia, Italy. e-mail: mathieu . fabredepf l.ch

Patrick Hild

Institut de Mathématiques de Toulouse - UMR CNRS 5219, Université Paul Sabatier, 118 route de Narbonne, 31062 Toulouse Cedex 9, France. e-mail: patrick.hildemath.univ-toulouse.fr

Rabii Mlika, Jérôme Pousin, Yves Renard

Université de Lyon, CNRS, INSA-Lyon, ICJ UMR5208, F-69621, Villeurbanne, France. e-mail: rabi i .mlika@insa-lyon. fr, jerome.pousindinsa-lyon.fr, yves.renardeinsa-lyon.fr 
Frictional contact problems can be formulated weakly within the framework of variational inequalities (see, e.g., $[40,36,64])$. Those are the very basis of most existing Finite Element Methods (FEM), see e.g. [64, 43, 51, 45, $68,90,89]$. For numerical computations with the FEM, various techniques have been devised to enforce contact and friction conditions at the discrete level, and the foremost are:

1. Penalty methods (see, e.g., $[65,78,77,64,20,24]$ ), where the set of inequations associated to contact is replaced with a non-linear inequation that approximates them. These methods remain primal, and are easy to implement. Nevertheless, consistency is lost, as a small amount of penetration, controlled by the penalty parameter, is allowed. Therefore the penalty parameter needs to be chosen with some care. Indeed, when the penalty parameter gets smaller to improve the approximation of contact conditions, the discrete problem gets stiffer and ill-conditioned, so iterative solvers such as generalized Newton may fail to converge.

2. Mixed methods (see, e.g., [51, 55, 10, 60, 67, 89]), where a Lagrange multiplier is introduced, that stands for the normal stress on the contact boundary (and for the tangential stress as well in case of frictional contact). The resulting weak form remains consistent, and characterizes the saddle-point of the corresponding Lagrangian. Inf-sup compatibility between the primal space of displacements and the dual space of Lagrange multipliers must be satisfied at the discrete level to ensure well-posedness: see, e.g., [89] and references therein for different solutions that have been proposed to overcome this issue. As usual for mixed formulations, stabilized finite element methods, such as Barbosa and Hughes stabilization [7], can be designed to circumvent the discrete inf-sup condition (see, e.g., [58]).

Nitsche's method has hardly been considered to discretize contact and friction conditions, despite the fact that it has gained popularity for other boundary conditions. The Nitsche method orginally proposed in $[75,5]$ aims at treating the boundary or interface conditions in a weak sense, with appropriate consistent terms that involve only the primal variables. It differs in this aspect from standard penalization techniques which are generally non-consistent [64]. Moreover, no additional unknown (Lagrange multiplier) is needed and, therefore, no discrete inf-sup condition must be fulfilled, contrarily to mixed methods (see, e.g., [51, 89]). Most of the applications of Nitsche's method during the last two decades involved linear conditions on the boundary of a domain or at the interface between subdomains: see, e.g,. [85] for the Dirichlet problem, [8] for domain decomposition with non-matching meshes and [47] for a global review. In some recent works [46, 54] it has been adapted for bilateral (persistent) contact, which still corresponds to linear boundary conditions on the contact zone. We remark furthermore that an algorithm for unilateral contact which makes use of Nitsche's method in its original form is presented and implemented in [46], and an extension to large strain bilateral contact has been performed in [91].

In $[23,28]$ a new Nitsche-based FEM was proposed and analyzed for Signorini's problem, where a linear elastic body is in frictionless contact with a rigid foundation. Conversely to bilateral (persistent) contact, Signorini's problem involves non-linear boundary conditions associated to unilateral contact, with an unknown actual contact region.

For this Nitsche-based FEM, optimal convergence in the $H^{1}(\Omega)$-norm of order $O\left(h^{\frac{1}{2}+v}\right)$ has been proved, provided the solution has a regularity $H^{\frac{3}{2}+v}(\Omega), 0<v \leq k-1 / 2(k=1,2$ is the polynomial degree of the Lagrange finite elements). To this purpose there is no need of additional assumption on the contact/friction zone, such as an increased regularity of the contact stress or a finite number of transition points between contact and non-contact. The proof applies in two-dimensional and three-dimensional cases, and for continuous affine and quadratic finite elements. Besides, the standard FEM for contact consists in a direct approximation of the variational inequality, with the elastic displacement as the only unknown. For this standard FEM and for many variants such as mixed/hybrid methods (e.g., [55, 10, 67]), stabilized mixed methods (e.g., [58]), penalty methods (e.g., [24]), it has been quite challenging to establish optimal convergence in the case the solution $\mathbf{u}$ belongs to $H^{\frac{3}{2}+v}(\Omega)(0<v \leq 1 / 2)$. As a matter of fact, the first fully optimal result, without extra assumptions, for the standard FEM has been achieved only recently, in 2015, see [35]. The first analyses in the 1970s were indeed sub-optimal with a convergence in $O\left(h^{\frac{1}{2}+\frac{v}{2}}\right)[84,49,51]$. In the 2000s, with additional assumptions on the finiteness of transition points between 
contact and non-contact optimality has been recovered (see [9] when $0<v<1 / 2$ and [60] when $v=1 / 2$ ). We refer to, e.g., $[60,89,59,35]$ for more detailed reviews on a priori error estimates for contact problems in elasticity. Moreover the Nitsche-based FEM encompasses symmetric and nonsymmetric variants depending upon a parameter called $\theta$. The symmetric case of [23] is recovered when $\theta=1$. When $\theta \neq 1$ positivity of the contact term in the Nitsche variational formulation is generally lost. Nevertheless some other advantages are recovered, mostly from the numerical viewpoint. Namely, one of the variants $(\theta=0)$ involves a reduced quantity of terms, which makes it easier to implement and to extend to contact problems involving non-linear elasticity. In addition, this nonsymmetric variant $\theta=0$ performs better in the sense it requires less Newton iterations to converge, for a wider range of the Nitsche parameter, than the variant $\theta=1$, see [81]. Concerning the skew-symmetric variant $\theta=-1$, the well-posedness of the discrete formulation and the optimal convergence are preserved irrespectively of the value of the Nitsche parameter. Note that for other boundary conditions, such as non-homogeneous Dirichlet, the symmetric variant $(\theta=1)$ as originally proposed by Nitsche [75] is the most widespread, since it preserves symmetry, and allows efficient solvers for linear systems with a symmetric matrix. However some nonsymmetric variants have been reconsidered recently, due to some remarkable robustness properties (see, e.g., [14, 11]). In the context of discontinuous Galerkin methods, such nonsymmetric variants are well-known as well (see, e.g., [33, Section 5.3.1, p.199]).

Lately, various extensions of the method proposed in $[23,28]$ have been carried out:

- An extension to Coulomb's friction has been formulated in [81] and tested numerically using a generalized Newton algorithm.

- An extension to Tresca's friction has been studied in [21]. Optimal convergence in $H^{1}(\Omega)$-norm has been established as well, without any assumption other than usual Sobolev regularity. For the standard FEM, and other methods, technical assumptions on the contact/friction set are needed to recover optimal convergence in $2 \mathrm{D}$, and in 3D, it remains an open issue [89].

- The case of contact in elastodynamics is dealt with in [26, 27]. As opposed to mixed methods, and identically as penalty and modified mass methods [63], Nitsche's discretization yields a well-posed semi-discrete problem in space (system of Lipschitz differential equations). It can be combined with various time-marching schemes, such as the theta-scheme, Newmark or a new "hybrid" scheme. The papers [26, 27] present a theoretical study of well-posedness and stability of the discretized schemes, as well as numerical experiments.

- The case of contact between two elastic bodies is addressed in [39, 29, 74]. In [39] Nitsche's method is combined with a cut-FEM / fictitious domain discretization, in the small deformations framework. In $[29,74]$ an unbiased variant implements the contact between two elastic bodies without making any difference between master and slave contact surfaces. The contact condition is the same on each surface. This is an advantage for treatment of self-contact or multi-body contact.

- Residual-based a posteriori error estimates are presented in [22]. Upper and lower bounds are proved under a saturation assumption, and the performance of the error estimates is investigated numerically.

- The topic of small-sliding frictional contact on 3D interfaces is the object of [3], where a weighted-Nitsche method is designed, and tested numerically.

- In [48] a least-square stabilized augmented lagrangian method, inspired by Nitsche's method, is described for unilateral contact. It shares some common features with Nitsche's method and allows increased flexibility on the discretization of the contact pressure. This has been followed recently by some papers $[17,19,16]$ that explore further the link between Nitsche and the augmented lagrangian, for the contact problem, the obstacle problem and interface problems with adhesive contact.

- A penalty-free Nitsche's method has been designed and studied in [18] for scalar Signorini's problem, that is an extension of the method studied in [14] for the Dirichlet problem. It is combined with a non-conforming discretization based on Crouzeix-Raviart finite elements. Stability and optimal convergence rate in $H^{1}(\Omega)$-norm are established. 
This paper is outlined as follows. In Section 2 we introduce a frictional contact problem and its Nitsche-based finite element approximation. The model problem that is focused on consists in unilateral contact between an elastic body and a rigid support (Signorini's problem), with Tresca's friction. We discuss as well the relationship with other methods, such as Barbosa and Hughes stabilized method, and the augmented lagrangian of Alart and Curnier. In Section 3 we summarize the main results that have been obtained regarding the consistency, well-posedness and convergence of the proposed FEM. Results of optimal convergence in $H^{1}(\Omega)$-norm are stated. Residual-based a posteriori error estimates are provided, as well as their main theoretical properties. Section 4 presents some numerical results of convergence for the $H^{1}(\Omega)$-norm of the displacement and for the contact condition, in the frictionless case, as well as some numerical results for the a posteriori error estimates. Some recent extensions are described in Section 5: contact between two elastic bodies, in small and large transformation frameworks, and contact in elastodynamics. Concluding remarks are given in Section 6.

Let us introduce some useful notations. In what follows, bold letters like $\mathbf{u}, \mathbf{v}$, indicate vector or tensor valued quantities, while the capital ones (e.g., $\mathbf{V}, \mathbf{K} \ldots$...) represent functional sets or operators involving vector fields. As usual, we denote by $\left(H^{s}(\cdot)\right)^{d}, s \in \mathbb{R}, d \in \mathbb{N}^{*}$ the Sobolev spaces in $d$ space dimensions (see [1]). The standard scalar product (resp. norm) of $\left(H^{s}(D)\right)^{d}$ is denoted by $(\cdot, \cdot)_{s, D}$ (resp. $\left.\|\cdot\|_{s, D}\right)$ and we keep the same notation for all the values of $d$. We use the same notations as in [64] for the Gâteaux derivative (or for the directional derivative) $\langle D F(v), w\rangle$ of a functional $F$ at point $v$ and in the direction $w$. The letter $C$ stands for a generic constant, independent of the discretization parameters.

\section{Setting and Nitsche-based method}

In this section we first present a frictional contact problem in 2.1. Its Nitsche-based finite element approximation is detailed in 2.2. We show in 2.3 that, for the problem under consideration, symmetric Nitsche formulation can be derived from an energy functional. Finally we discuss the relationship with other methods in 2.4: Nitsche for Dirichlet boundary conditions, Barbosa and Hughes stabilized method and the augmented lagrangian of Alart and Curnier.

\subsection{Unilateral contact with Tresca friction}

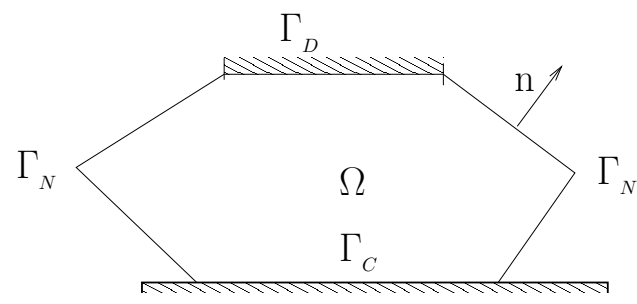

Fig. 1 Elastic body that occupies the domain $\Omega$. The boundary $\partial \Omega$ is divided into three non-overlapping parts: $\Gamma_{D}$ (the body is clamped), $\Gamma_{N}$ (tractions are imposed) and $\Gamma_{C}$ (contact boundary). 
We consider an elastic body whose reference configuration is represented by the domain $\Omega$ in $\mathbb{R}^{d}$ with $d=2$ or $d=3$ (see Fig. 1 when $d=2$ ). Small strain assumption is made, as well as plane strain when $d=2$. The boundary $\partial \Omega$ of $\Omega$ is polygonal or polyhedral and we partition $\partial \Omega$ in three nonoverlapping parts $\Gamma_{D}, \Gamma_{N}$ and the (potential) contact/friction boundary $\Gamma_{C}$, with meas $\left(\Gamma_{D}\right)>0$ and meas $\left(\Gamma_{C}\right)>0$. The contact/friction boundary is supposed to be a straight line segment when $d=2$ or a planar polygon when $d=3$ to simplify. The normal unit outward vector on $\partial \Omega$ is denoted $\mathbf{n}$. The body is clamped on $\Gamma_{D}$ for the sake of simplicity. It is subjected to volume forces $\mathbf{f} \in\left(L^{2}(\Omega)\right)^{d}$ and to surface loads $\mathbf{f}_{N} \in\left(L^{2}\left(\Gamma_{N}\right)\right)^{d}$.

The unilateral contact problem with Tresca friction under consideration consists in finding the displacement field $\mathbf{u}: \Omega \rightarrow \mathbb{R}^{d}$ verifying the equations and conditions (1)-(2)-(3):

$$
\begin{array}{rrrr}
\operatorname{div} \sigma(\mathbf{u})+\mathbf{f}=\mathbf{0} & \text { in } \Omega, & \sigma(\mathbf{u})=\mathbf{C} \varepsilon(\mathbf{u}) & \text { in } \Omega, \\
\mathbf{u}=\mathbf{0} & \text { on } \Gamma_{D}, & \sigma(\mathbf{u}) \mathbf{n}=\mathbf{f}_{N} & \text { on } \Gamma_{N},
\end{array}
$$

where $\sigma=\left(\sigma_{i j}\right), 1 \leq i, j \leq d$, stands for the stress tensor field and div denotes the divergence operator of tensor valued functions. The notation $\varepsilon(\mathbf{v})=\left(\nabla \mathbf{v}+\nabla \mathbf{v}^{T}\right) / 2$ represents the linearized strain tensor field and $\mathbf{C}$ is the fourth order symmetric elasticity tensor having the usual uniform ellipticity and boundedness property. For any displacement field $\mathbf{v}$ and for any density of surface forces $\sigma(\mathbf{v}) \mathbf{n}$ defined on $\partial \Omega$ we adopt the following decomposition into normal and tangential components:

$$
\mathbf{v}=v_{n} \mathbf{n}+\mathbf{v}_{\mathbf{t}} \quad \text { and } \quad \sigma(\mathbf{v}) \mathbf{n}=\sigma_{n}(\mathbf{v}) \mathbf{n}+\sigma_{\mathbf{t}}(\mathbf{v}) .
$$

The unilateral contact conditions (Kuhn-Tucker conditions) on $\Gamma_{C}$ are formulated as follows:

$$
u_{n} \leq 0, \quad \sigma_{n}(\mathbf{u}) \leq 0, \quad \sigma_{n}(\mathbf{u}) u_{n}=0 .
$$

Let $s \in L^{2}\left(\Gamma_{C}\right), s \geq 0$ be a given threshold. The Tresca friction condition on $\Gamma_{C}$ reads:

$$
\left\{\begin{array}{llrl}
\left|\sigma_{\mathbf{t}}(\mathbf{u})\right| & \leq s & \text { if } \mathbf{u}_{\mathbf{t}}=\mathbf{0}, & (i) \\
\sigma_{\mathbf{t}}(\mathbf{u}) & =-s \frac{\mathbf{u}_{\mathbf{t}}}{\left|\mathbf{u}_{\mathbf{t}}\right|} & \text { otherwise, } & (i i)
\end{array}\right.
$$

where $|\cdot|$ stands for the euclidean norm in $\mathbb{R}^{d-1}$. Note that conditions (3)-(i) and (3)-(ii) imply that $\left|\sigma_{\mathbf{t}}(\mathbf{u})\right| \leq s$ in all cases, and that if $\left|\sigma_{\mathbf{t}}(\mathbf{u})\right|<s$, we must have $\mathbf{u}_{\mathbf{t}}=\mathbf{0}$.

Remark 1. The case of bilateral contact with Tresca friction can be considered too, simply substituting to equations (2) the following one on $\Gamma_{C}$ :

$$
u_{n}=0
$$

The case of frictionless contact is recovered setting $s=0$ in (3).

Remark 2. The conditions of (static) Coulomb friction can be written similarly as:

$$
\left\{\begin{array}{llrl}
\left|\sigma_{\mathbf{t}}(\mathbf{u})\right| & \leq-\mathscr{F} \sigma_{n}(\mathbf{u}) & \text { if } \mathbf{u}_{\mathbf{t}}=\mathbf{0}, & (i) \\
\sigma_{\mathbf{t}}(\mathbf{u}) & =\mathscr{F} \sigma_{n}(\mathbf{u}) \frac{\mathbf{u}_{\mathbf{t}}}{\left|\mathbf{u}_{\mathbf{t}}\right|} & \text { otherwise, } & (i i)
\end{array}\right.
$$

where $\mathscr{F} \geq 0$ is the friction coefficient. Conditions (5) mean that, at a given contact point on $\Gamma_{C}$, sliding can not occur while the magnitude of the tangential stress $\left|\sigma_{\mathbf{t}}(\mathbf{u})\right|$ is strictly below the threshold $-\mathscr{F} \sigma_{n}(\mathbf{u})$. When the threshold $-\mathscr{F} \sigma_{n}(\mathbf{u})$ is reached, sliding may happen, in a direction opposite to $\sigma_{\mathbf{t}}(\mathbf{u})$ (see, e.g., [64, Chapter 10]). Remark that this static form of Coulomb friction is an adaptation of the quasi-static (or dynamic) Coulomb's law, 
in which the tangential velocity $\dot{\mathbf{u}}_{\mathbf{t}}$ plays the same role as the displacement $\mathbf{u}_{\mathbf{t}}$ (see formulation (63), and, e.g., $[36,37,6])$. A formulation such as (5) is obtained for instance when the quasi-static Coulomb's law is discretized with a time-marching scheme (see, e.g., [89]).

Remark 3. In the Tresca friction model (3), it is assumed that the amplitude of the normal friction threshold is known (i.e., $\mathscr{F}\left|\sigma_{n}(\mathbf{u})\right|=s$, see, e.g., [64, Section 10.3]). The introduction of the Tresca friction model is motivated more by its mathematical simplicity than by physical reasons, though it can be of use in special situations. For instance:

- when a thin belt/tape is pressed against an elastic body, with a known pressure (see, e.g., [64, Chapter 10]),

- for persistent contact between solids with high intensity of contact pressures, such as it may occur in earth sciences (see, e.g., [79]).

Moreover the Tresca friction model can be useful for instance when Coulomb friction is approximated iteratively (see, e.g., [50] and [67, Section 9, Theorem 7]).

We introduce the Hilbert space $\mathbf{V}$ and the convex cone $\mathbf{K}$ of admissible displacements which satisfy the nonpenetration condition on the contact zone $\Gamma_{C}$ :

$$
\mathbf{V}:=\left\{\mathbf{v} \in\left(H^{1}(\Omega)\right)^{d}: \mathbf{v}=\mathbf{0} \text { on } \Gamma_{D}\right\}, \quad \mathbf{K}:=\left\{\mathbf{v} \in \mathbf{V}: v_{n}=\mathbf{v} \cdot \mathbf{n} \leq 0 \text { on } \Gamma_{C}\right\} .
$$

Define

$$
a(\mathbf{u}, \mathbf{v}):=\int_{\Omega} \sigma(\mathbf{u}): \varepsilon(\mathbf{v}) d \Omega, \quad L(\mathbf{v}):=\int_{\Omega} \mathbf{f} \cdot \mathbf{v} d \Omega+\int_{\Gamma_{N}} \mathbf{f}_{N} \cdot \mathbf{v} d \Gamma, \quad j(\mathbf{v}):=\int_{\Gamma_{C}} s\left|\mathbf{v}_{\mathbf{t}}\right| d \Gamma,
$$

for any $\mathbf{u}$ and $\mathbf{v}$ in $\mathbf{V}$.

The weak formulation of Problem (1)-(3) as a variational inequality of the second kind is:

$$
\left\{\begin{array}{l}
\text { Find } \mathbf{u} \in \mathbf{K} \text { such that: } \\
a(\mathbf{u}, \mathbf{v}-\mathbf{u})+j(\mathbf{v})-j(\mathbf{u}) \geq L(\mathbf{v}-\mathbf{u}), \quad \forall \mathbf{v} \in \mathbf{K} .
\end{array}\right.
$$

which admits a unique solution (see, e.g., [42, Theorem 5.1, Remark 5.2, p.69]). Moreover this solution is the unique minimizer on $\mathbf{K}$ of the functional

$$
\mathscr{J}: \mathbf{V} \ni \mathbf{v} \mapsto \frac{1}{2} a(\mathbf{v}, \mathbf{v})-L(\mathbf{v})+j(\mathbf{v}) \in \mathbb{R} .
$$

Remark 4. In the case of bilateral contact (condition (4) instead of (2)), the same weak formulation (6) holds, replacing the convex cone $\mathbf{K}$ by the space:

$$
\mathbf{V}_{\mathrm{b}}:=\left\{\mathbf{v} \in \mathbf{V}: v_{n}=0 \text { on } \Gamma_{C}\right\} .
$$

\subsection{The Nitsche-based finite element method}

Let $\mathbf{V}^{h} \subset \mathbf{V}$ be a family of finite dimensional vector spaces (see [30, 38, 12]) indexed by $h$ coming from a family $\mathscr{T}^{h}$ of triangulations of the domain $\Omega\left(h=\max _{T \in \mathscr{T}^{h}} h_{T}\right.$ where $h_{T}$ is the diameter of $\left.T\right)$. We suppose that the family of triangulations is regular, i.e., there exists $\sigma>0$ such that $\forall T \in \mathscr{T}^{h}, h_{T} / \rho_{T} \leq \sigma$ where $\rho_{T}$ denotes the radius of the inscribed ball in $T$. Furthermore we suppose that this family is conformal to the subdivision of the 
boundary into $\Gamma_{D}, \Gamma_{N}$ and $\Gamma_{C}$ (i.e., a face of an element $T \in \mathscr{T}^{h}$ is not allowed to have simultaneous non-empty intersection with more than one part of the subdivision). We choose a standard Lagrange finite element method of degree $k$ with $k=1$ or $k=2$, i.e.:

$$
\mathbf{V}^{h}:=\left\{\mathbf{v}^{h} \in\left(\mathscr{C}^{0}(\bar{\Omega})\right)^{d}: \mathbf{v}^{h}{ }_{T} \in\left(\mathbb{P}_{k}(T)\right)^{d}, \forall T \in \mathscr{T}^{h}, \mathbf{v}^{h}=\mathbf{0} \text { on } \Gamma_{D}\right\}
$$

However, the analysis would be similar for any $\mathscr{C}^{0}$-conforming finite element method.

We make use of the notation $[\cdot]_{\mathbb{R}^{-}}$, that stands for the projection onto $\mathbb{R}^{-}\left([x]_{\mathbb{R}^{-}}=\frac{1}{2}(x-|x|)\right.$ for $\left.x \in \mathbb{R}\right)$. Moreover, for any $\alpha \in \mathbb{R}^{+}$, we introduce the notation $[\cdot]_{\alpha}$ for the orthogonal projection onto $\mathscr{B}(\mathbf{0}, \alpha) \subset \mathbb{R}^{d-1}$, where $\mathscr{B}(\mathbf{0}, \alpha)$ is the closed ball centered at the origin $\mathbf{0}$ and of radius $\alpha$. This operation can be defined analytically, for $\mathbf{x} \in \mathbb{R}^{d-1}$ by:

$$
[\mathbf{x}]_{\alpha}=\left\{\begin{array}{cc}
\mathbf{x} & \text { if }|\mathbf{x}| \leq \alpha \\
\alpha \frac{\mathbf{x}}{|\mathbf{x}|} & \text { otherwise }
\end{array}\right.
$$

The notation $H(\cdot)$ will stand for a "Heaviside" function: for any $x \in \mathbb{R}$,

$$
H(x):= \begin{cases}1 & \text { if } x>0 \\ \frac{1}{2} & \text { if } x=0 \\ 0 & \text { if } x<0\end{cases}
$$

We adopt the convention $H(0)=1 / 2$ to allow the property $H(x)+H(-x)=1, \forall x \in \mathbb{R}$. Moreover we will make use of the property

$$
H(-x)[x]_{\mathbb{R}^{-}}=[x]_{\mathbb{R}^{-}}, \quad \forall x \in \mathbb{R} .
$$

The next properties of projections are useful in the mathematical analysis of the method:

$$
\begin{array}{ll}
(y-x)\left([y]_{\mathbb{R}^{-}}-[x]_{\mathbb{R}^{-}}\right) \geq\left([y]_{\mathbb{R}^{-}}-[x]_{\mathbb{R}^{-}}\right)^{2} & \forall x, y \in \mathbb{R}, \\
(\mathbf{y}-\mathbf{x}) \cdot\left([\mathbf{y}]_{\alpha}-[\mathbf{x}]_{\alpha}\right) \geq\left|[\mathbf{y}]_{\alpha}-[\mathbf{x}]_{\alpha}\right|^{2} & \forall \mathbf{x}, \mathbf{y} \in \mathbb{R}^{d-1},
\end{array}
$$

where $\cdot$ is the euclidean scalar product in $\mathbb{R}^{d-1}$.

The next result has been pointed out earlier in [2] (see as well [23, 21] for detailed formal proofs).

Proposition 1. Let $\gamma$ be a positive function defined on $\Gamma_{C}$. The contact with Tresca friction conditions (2)-(3) can be reformulated as follows:

$$
\begin{aligned}
& \sigma_{n}(\mathbf{u})=\left[\sigma_{n}(\mathbf{u})-\gamma u_{n}\right]_{\mathbb{R}^{-}}, \\
& \sigma_{\mathbf{t}}(\mathbf{u})=\left[\sigma_{\mathbf{t}}(\mathbf{u})-\gamma \mathbf{u}_{\mathbf{t}}\right]_{s} .
\end{aligned}
$$

Remark 5. Equation (12) is an example of nonlinear complementarity (NCP) function that allows to reformulate complementarity conditions such as expressed in (2) using a single nonlinear relationship (see, e.g., [41] and references therein). This is not the unique possible formulation, but is among the simplest ones.

Let now $\theta \in \mathbb{R}$ be a fixed parameter and $\gamma$ a positive function defined on $\Gamma_{C}$. Let $\mathbf{u}$ be the solution of the unilateral contact problem in its strong form (1)-(2)-(3). We assume that $\mathbf{u}$ is sufficiently regular so that all the following calculations make sense. From the Green formula and equation (1), we get for every $\mathbf{v} \in \mathbf{V}$ :

$$
a(\mathbf{u}, \mathbf{v})-\int_{\Gamma_{C}} \sigma_{n}(\mathbf{u}) v_{n} d \Gamma-\int_{\Gamma_{C}} \sigma_{\mathbf{t}}(\mathbf{u}) \cdot \mathbf{v}_{\mathbf{t}} d \Gamma=L(\mathbf{v}) .
$$


Note that

$$
\begin{aligned}
& v_{n}=-\frac{1}{\gamma}\left(\theta \sigma_{n}(\mathbf{v})-\gamma v_{n}\right)+\frac{\theta}{\gamma} \sigma_{n}(\mathbf{v}) \\
& \mathbf{v}_{\mathbf{t}}=-\frac{1}{\gamma}\left(\theta \sigma_{\mathbf{t}}(\mathbf{v})-\gamma \mathbf{v}_{\mathbf{t}}\right)+\frac{\theta}{\gamma} \sigma_{\mathbf{t}}(\mathbf{v})
\end{aligned}
$$

So:

$$
\begin{aligned}
a(\mathbf{u}, \mathbf{v}) & -\int_{\Gamma_{C}} \frac{\theta}{\gamma} \sigma_{n}(\mathbf{u}) \sigma_{n}(\mathbf{v}) d \Gamma+\int_{\Gamma_{C}} \frac{1}{\gamma} \sigma_{n}(\mathbf{u})\left(\theta \sigma_{n}(\mathbf{v})-\gamma v_{n}\right) d \Gamma \\
& -\int_{\Gamma_{C}} \frac{\theta}{\gamma} \sigma_{\mathbf{t}}(\mathbf{u}) \cdot \sigma_{\mathbf{t}}(\mathbf{v}) d \Gamma+\int_{\Gamma_{C}} \frac{1}{\gamma} \sigma_{\mathbf{t}}(\mathbf{u}) \cdot\left(\theta \sigma_{\mathbf{t}}(\mathbf{v})-\gamma \mathbf{v}_{\mathbf{t}}\right) d \Gamma=L(\mathbf{v}) .
\end{aligned}
$$

Finally, using conditions (12) and (13), we obtain:

$$
\begin{aligned}
a(\mathbf{u}, \mathbf{v}) & -\int_{\Gamma_{C}} \frac{\theta}{\gamma} \sigma_{n}(\mathbf{u}) \sigma_{n}(\mathbf{v}) d \Gamma+\int_{\Gamma_{C}} \frac{1}{\gamma}\left[\sigma_{n}(\mathbf{u})-\gamma u_{n}\right]_{\mathbb{R}^{-}}\left(\theta \sigma_{n}(\mathbf{v})-\gamma v_{n}\right) d \Gamma \\
& -\int_{\Gamma_{C}} \frac{\theta}{\gamma} \sigma_{\mathbf{t}}(\mathbf{u}) \cdot \sigma_{\mathbf{t}}(\mathbf{v}) d \Gamma+\int_{\Gamma_{C}} \frac{1}{\gamma}\left[\sigma_{\mathbf{t}}(\mathbf{u})-\gamma \mathbf{u}_{\mathbf{t}}\right]_{S} \cdot\left(\theta \sigma_{\mathbf{t}}(\mathbf{v})-\gamma \mathbf{v}_{\mathbf{t}}\right) d \Gamma=L(\mathbf{v})
\end{aligned}
$$

Formula (14) is the starting point of the Nitsche-based formulation. We remark that it may have no sense at the continuous level if $\mathbf{u}$ lacks of regularity (the only assumption $\mathbf{u} \in \mathbf{V}$ is not sufficient to justify the above calculations). Nevertheless we consider in what follows that $\gamma$ is a positive piecewise constant function on the contact and friction interface $\Gamma_{C}$ which satisfies

$$
\left.\gamma\right|_{T \cap \Gamma_{C}}=\frac{\gamma_{0}}{h_{T}}
$$

for every $T$ that has a non-empty intersection of dimension $d-1$ with $\Gamma_{C}$, and where $\gamma_{0}$ is a positive given constant (the Nitsche parameter). Note that the value of $\gamma$ on element intersections has no influence. Let us introduce the discrete linear operators

$$
\mathrm{P}_{\theta, \gamma}^{\mathbf{n}}: \begin{aligned}
& \mathbf{V}^{h} \rightarrow \quad L^{2}\left(\Gamma_{C}\right) \\
& \mathbf{v}^{h} \mapsto \theta \sigma_{n}\left(\mathbf{v}^{h}\right)-\gamma v_{n}^{h}
\end{aligned} \quad \text { and } \quad \mathbf{P}_{\theta, \gamma}^{\mathbf{t}}: \begin{aligned}
& \mathbf{V}^{h} \rightarrow\left(L^{2}\left(\Gamma_{C}\right)\right)^{d-1} \\
& \mathbf{v}^{h} \mapsto \theta \sigma_{t}\left(\mathbf{v}^{h}\right)-\gamma \mathbf{v}_{\mathbf{t}}^{h}
\end{aligned}
$$

Define as well the bilinear form:

$$
A_{\theta \gamma}\left(\mathbf{u}^{h}, \mathbf{v}^{h}\right):=a\left(\mathbf{u}^{h}, \mathbf{v}^{h}\right)-\int_{\Gamma_{C}} \frac{\theta}{\gamma} \sigma\left(\mathbf{u}^{h}\right) \mathbf{n} \cdot \sigma\left(\mathbf{v}^{h}\right) \mathbf{n} d \Gamma
$$

The Nitsche-based method for unilateral contact with Tresca friction then reads:

$$
\left\{\begin{array}{l}
\text { Find } \mathbf{u}^{h} \in \mathbf{V}^{h} \text { such that: } \\
A_{\theta \gamma}\left(\mathbf{u}^{h}, \mathbf{v}^{h}\right)+\int_{\Gamma_{C}} \frac{1}{\gamma}\left[\mathrm{P}_{1, \gamma}^{\mathbf{n}}\left(\mathbf{u}^{h}\right)\right]_{\mathbb{R}^{-}} \mathrm{P}_{\theta, \gamma}^{\mathbf{n}}\left(\mathbf{v}^{h}\right) d \Gamma+\int_{\Gamma_{C}} \frac{1}{\gamma}\left[\mathbf{P}_{1, \gamma}^{\mathbf{t}}\left(\mathbf{u}^{h}\right)\right]_{s} \cdot \mathbf{P}_{\theta, \gamma}^{\mathbf{t}}\left(\mathbf{v}^{h}\right) d \Gamma=L\left(\mathbf{v}^{h}\right), \quad \forall \mathbf{v}^{h} \in \mathbf{V}^{h} .
\end{array}\right.
$$

Note that we adopted in this presentation a different convention for notations compared to previous works [23, 28, 21]. This is in order to get closer to the formulations provided in most of the papers on Nitsche's method and on 
the augmented lagrangian method (see Section 2.4). Furthermore, and as already stated in [28] the parameter $\theta$ can be set to some particular values, namely:

1. for $\theta=1$ we recover a symmetric method for which the contact term

$$
\int_{\Gamma_{C}} \frac{1}{\gamma}\left[\mathrm{P}_{1, \gamma}^{\mathbf{n}}\left(\mathbf{u}^{h}\right)\right]_{\mathbb{R}^{-}} \mathrm{P}_{1, \gamma}^{\mathbf{n}}\left(\mathbf{v}^{h}\right) d \Gamma
$$

is positive when we set $\mathbf{v}^{h}=\mathbf{u}^{h}$. This method can be derived from an energy functional (see Section 2.3). The tangent matrix resulting from a linearization with generalized Newton is symmetric.

2. for $\theta=0$ we recover a simple method close to penalty and to the augmented lagrangian. It involves only a few terms and is of easiest implementation.

3. for $\theta=-1$ the skew-symmetric method admits one unique solution and converges optimally irrespectively of the value of the Nitsche parameter $\gamma_{0}>0$.

Remark 6. The operators $\mathbf{P}_{\theta, \gamma}^{\mathbf{n}}(\cdot)$ and $\mathbf{P}_{\theta, \gamma}^{\mathbf{t}}(\cdot)$ have no special physical meaning but can be related to proximal augmented lagrangian formulations (see 2.4.3 for more details).

Remark 7. For frictionless unilateral contact $(s=0$ in (3)) we can follow the same path as previously for the derivation of the method. With the solution $\mathbf{u}$ to (1)-(2)-(3) and the condition $\sigma_{\mathbf{t}}(\mathbf{u})=\mathbf{0}$ there holds first for every $\mathbf{v} \in \mathbf{V}:$

$$
a(\mathbf{u}, \mathbf{v})-\int_{\Gamma_{C}} \sigma_{n}(\mathbf{u}) v_{n} d \Gamma=L(\mathbf{v})
$$

and then

$$
a(\mathbf{u}, \mathbf{v})-\int_{\Gamma_{C}} \frac{\theta}{\gamma} \sigma_{n}(\mathbf{u}) \sigma_{n}(\mathbf{v}) d \Gamma+\int_{\Gamma_{C}} \frac{1}{\gamma} \sigma_{n}(\mathbf{u})\left(\theta \sigma_{n}(\mathbf{v})-\gamma v_{n}\right) d \Gamma=L(\mathbf{v})
$$

Using condition (12) we obtain:

$$
a(\mathbf{u}, \mathbf{v})-\int_{\Gamma_{C}} \frac{\theta}{\gamma} \sigma_{n}(\mathbf{u}) \sigma_{n}(\mathbf{v}) d \Gamma+\int_{\Gamma_{C}} \frac{1}{\gamma}\left[\sigma_{n}(\mathbf{u})-\gamma u_{n}\right]_{\mathbb{R}^{-}}\left(\theta \sigma_{n}(\mathbf{v})-\gamma v_{n}\right) d \Gamma=L(\mathbf{v}) .
$$

As a result, the counterpart of (16) reads:

$$
\left\{\begin{array}{l}
\text { Find } \mathbf{u}^{h} \in \mathbf{V}^{h} \text { such that: } \\
A_{\theta \gamma}^{\mathbf{n}}\left(\mathbf{u}^{h}, \mathbf{v}^{h}\right)+\int_{\Gamma_{C}} \frac{1}{\gamma}\left[\mathrm{P}_{1, \gamma}^{\mathbf{n}}\left(\mathbf{u}^{h}\right)\right]_{\mathbb{R}^{-}} \mathrm{P}_{\theta, \gamma}^{\mathbf{n}}\left(\mathbf{v}^{h}\right) d \Gamma=L\left(\mathbf{v}^{h}\right), \quad \forall \mathbf{v}^{h} \in \mathbf{V}^{h},
\end{array}\right.
$$

where

$$
A_{\theta \gamma}^{\mathbf{n}}\left(\mathbf{u}^{h}, \mathbf{v}^{h}\right):=a\left(\mathbf{u}^{h}, \mathbf{v}^{h}\right)-\int_{\Gamma_{C}} \frac{\theta}{\gamma} \sigma_{n}\left(\mathbf{u}^{h}\right) \sigma_{n}\left(\mathbf{v}^{h}\right) d \Gamma
$$

Remark that setting $s=0$ in formulation (16) does not lead to formulation (17) because of the difference between $A_{\theta \gamma}(\cdot, \cdot)$ and $A_{\theta \gamma}^{\mathbf{n}}(\cdot, \cdot)$.

Remark 8. For bilateral contact with friction (equations (1)-(3)-(4)), the Nitsche-based formulation reads:

$$
\left\{\begin{array}{l}
\text { Find } \mathbf{u}^{h} \in \mathbf{V}_{\mathrm{b}}^{h} \text { such that: } \\
A_{\theta \gamma}^{\mathbf{t}}\left(\mathbf{u}^{h}, \mathbf{v}^{h}\right)+\int_{\Gamma_{C}} \frac{1}{\gamma}\left[\mathbf{P}_{1, \gamma}^{\mathbf{t}}\left(\mathbf{u}^{h}\right)\right]_{s} \cdot \mathbf{P}_{\theta, \gamma}^{\mathbf{t}}\left(\mathbf{v}^{h}\right) d \Gamma=L\left(\mathbf{v}^{h}\right), \quad \forall \mathbf{v}^{h} \in \mathbf{V}_{\mathrm{b}}^{h}
\end{array}\right.
$$


where $A_{\theta \gamma}^{\mathbf{t}}\left(\mathbf{u}^{h}, \mathbf{v}^{h}\right):=a\left(\mathbf{u}^{h}, \mathbf{v}^{h}\right)-\int_{\Gamma_{C}} \frac{\theta}{\gamma} \sigma_{\mathbf{t}}\left(\mathbf{u}^{h}\right) \cdot \sigma_{\mathbf{t}}\left(\mathbf{v}^{h}\right) d \Gamma$ and $\mathbf{V}_{b}^{h}:=\mathbf{V}^{h} \cap \mathbf{V}_{\mathrm{b}}$.

Remark 9. Following the same path as in Proposition 1 the Coulomb friction conditions (5) can be reformulated as:

$$
\sigma_{\mathbf{t}}(\mathbf{u})=\left[\sigma_{\mathbf{t}}(\mathbf{u})-\gamma \mathbf{u}_{\mathbf{t}}\right]_{\left(-\mathscr{F} \sigma_{n}(\mathbf{u})\right)}=\left[\sigma_{\mathbf{t}}(\mathbf{u})-\gamma \mathbf{u}_{\mathbf{t}}\right]_{\left(-\mathscr{F}\left[\sigma_{n}(\mathbf{u})-\gamma u_{n}\right]_{\mathbb{R}^{-}}\right)} .
$$

This motivates the introduction of the following Nitsche-based formulation for unilateral contact with Coulomb friction (equations (1)-(2)-(5)):

$$
\left\{\begin{array}{l}
\text { Find } \mathbf{u}^{h} \in \mathbf{V}^{h} \text { such that: } \\
A_{\theta \gamma}\left(\mathbf{u}^{h}, \mathbf{v}^{h}\right)+\int_{\Gamma_{C}} \frac{1}{\gamma}\left[\mathrm{P}_{1, \gamma}^{\mathbf{n}}\left(\mathbf{u}^{h}\right)\right]_{\mathbb{R}^{-}} \mathbf{P}_{\theta, \gamma}^{\mathbf{n}}\left(\mathbf{v}^{h}\right) d \Gamma \\
+\int_{\Gamma_{C}} \frac{1}{\gamma}\left[\mathbf{P}_{1, \gamma}^{\mathbf{t}}\left(\mathbf{u}^{h}\right)\right]\left(-\mathscr{F}\left[\mathrm{P}_{1, \gamma}^{\mathbf{n}}\left(\mathbf{u}^{h}\right)\right]_{\mathbb{R}^{-}}\right) \\
\cdot \mathbf{P}_{\theta, \gamma}^{\mathbf{t}}\left(\mathbf{v}^{h}\right) d \Gamma=L\left(\mathbf{v}^{h}\right), \quad \forall \mathbf{v}^{h} \in \mathbf{V}^{h} .
\end{array}\right.
$$

We next define convenient mesh-dependent norms, in fact weighted $L^{2}\left(\Gamma_{C}\right)$-norm (since $\left.\left.\left(\gamma_{0} / \gamma\right)\right|_{T}=h_{T}\right)$.

Definition 1. For any $v \in L^{2}\left(\Gamma_{C}\right)$, we set

$$
\|v\|_{-\frac{1}{2}, h, \Gamma_{C}}:=\left\|\left(\gamma_{0} / \gamma\right)^{\frac{1}{2}} v\right\|_{0, \Gamma_{C}}, \quad\|v\|_{\frac{1}{2}, h, \Gamma_{C}}:=\left\|\left(\gamma / \gamma_{0}\right)^{\frac{1}{2}} v\right\|_{0, \Gamma_{C}} .
$$

The same definitions extend straightforwardly to functions in $\left(L^{2}\left(\Gamma_{C}\right)\right)^{d-1}$.

Additionally, it will be sometimes convenient to endow $\mathbf{V}^{h}$ with the following mesh- and parameter-dependent scalar product:

Definition 2. For all $\mathbf{v}^{h}, \mathbf{w}^{h} \in \mathbf{V}^{h}$ we set

$$
\left(\mathbf{v}^{h}, \mathbf{w}^{h}\right)_{\gamma}:=\left(\mathbf{v}^{h}, \mathbf{w}^{h}\right)_{1, \Omega}+\left(\gamma^{\frac{1}{2}} \mathbf{v}^{h}, \gamma^{\frac{1}{2}} \mathbf{w}^{h}\right)_{0, \Gamma_{C}},
$$

and note $\|\cdot\|_{\gamma}:=(\cdot, \cdot)_{\gamma}^{\frac{1}{2}}$ the corresponding norm. Remark that the two norms $\|\cdot\|_{\gamma}$ and $\|\cdot\|_{1, \Omega}$ are equivalent on $\mathbf{V}^{h}$, in the following sense (for a quasi-uniform mesh $\mathscr{T}^{h}$ ):

$$
\left\|\mathbf{v}^{h}\right\|_{1, \Omega} \leq\left\|\mathbf{v}^{h}\right\|_{\gamma} \leq\left(1+C \frac{\gamma_{0}}{h}\right)\left\|\mathbf{v}^{h}\right\|_{1, \Omega}
$$

for any $\mathbf{v}^{h} \in \mathbf{V}^{h}$. The positive constant $C$ comes from the trace inequality and the constant of quasi-uniformity of the mesh $\mathscr{T}^{h}$. For a mesh $\mathscr{T}^{h}$ that is not quasi-uniform, the same relationship holds, replacing $h$ by $\left(\min _{T \in \mathscr{T}^{h}} h_{T}\right)$.

We end this section with the following statement: a discrete trace inequality (see, e.g., [86, 21]), that is a key ingredient for the whole mathematical analysis of Nitsche's based methods.

Lemma 1. There exists $C>0$, independent of the parameter $\gamma_{0}$ and of the mesh size $h$, such that, for all $\mathbf{v}^{h} \in \mathbf{V}^{h}$ :

$$
\left\|\sigma_{n}\left(\mathbf{v}^{h}\right)\right\|_{-\frac{1}{2}, h, \Gamma_{C}}+\left\|\sigma_{\mathbf{t}}\left(\mathbf{v}^{h}\right)\right\|_{-\frac{1}{2}, h, \Gamma_{C}} \leq C\left\|\mathbf{v}^{h}\right\|_{1, \Omega} .
$$




\subsection{Energy minimization for the symmetric variant}

We show in this section that an energy functional can be associated to Problem (16) in the symmetric case $(\theta=1)$, that is a discrete counterpart of $\mathscr{J}(\cdot)$. Using Riesz's representation theorem, we identify $\left(\mathbf{V}^{h},(\cdot, \cdot)_{\gamma}\right)$ to its dual. Let us first introduce a functional for the total potential energy, i.e. the strain energy and the potential energy of the external forces:

$$
\mathscr{J}_{\mathrm{E}}\left(\mathbf{v}^{h}\right):=\frac{1}{2} a\left(\mathbf{v}^{h}, \mathbf{v}^{h}\right)-L\left(\mathbf{v}^{h}\right),
$$

for any $\mathbf{v}^{h} \in \mathbf{V}^{h}$. For the contact condition (12) we add the term:

$$
\mathscr{J}_{\gamma}^{\mathbf{n}}\left(\mathbf{v}^{h}\right):=\frac{1}{2} \int_{\Gamma_{C}} \frac{1}{\gamma}\left[\mathrm{P}_{1, \gamma}^{\mathbf{n}}\left(\mathbf{v}^{h}\right)\right]_{\mathbb{R}^{-}}^{2} d \Gamma-\frac{1}{2} \int_{\Gamma_{C}} \frac{1}{\gamma} \sigma_{n}\left(\mathbf{v}^{h}\right)^{2} d \Gamma
$$

And for the Tresca friction condition (13) we take:

$$
\mathscr{J}_{\gamma}^{\mathbf{t}}\left(\mathbf{v}^{h}\right):=-\frac{1}{2} \int_{\Gamma_{C}} \frac{1}{\gamma}\left|\mathbf{P}_{1, \gamma}^{\mathbf{t}}\left(\mathbf{v}^{h}\right)-\left[\mathbf{P}_{1, \gamma}^{\mathbf{t}}\left(\mathbf{v}^{h}\right)\right]_{s}\right|^{2} d \Gamma+\frac{1}{2} \int_{\Gamma_{C}} \frac{1}{\gamma}\left|\mathbf{P}_{1, \gamma}^{\mathbf{t}}\left(\mathbf{v}^{h}\right)\right|^{2} d \Gamma-\frac{1}{2} \int_{\Gamma_{C}} \frac{1}{\gamma}\left|\sigma_{\mathbf{t}}\left(\mathbf{v}^{h}\right)\right|^{2} d \Gamma .
$$

The energy functional associated to Problem (16) is then:

$$
\mathscr{J}_{\gamma}:=\mathscr{J}_{\mathrm{E}}+\mathscr{J}_{\gamma}^{\mathbf{n}}+\mathscr{J}_{\gamma}^{\mathbf{t}}
$$

Now, when $\theta=1$, we are able to characterize Problem (16) as the first-order optimality condition associated to the minimization of $\mathscr{J}_{\gamma}(\cdot)$ on $\mathbf{V}^{h}$ :

Proposition 2. Suppose that $\theta=1$ and that $\gamma_{0}$ is large enough. Then:

1. $\left(\mathscr{J}_{\gamma}+L\right)(\cdot)$ is non-negative.

2. $\mathscr{J}_{\gamma}(\cdot)$ is a Gâteaux-differentiable and convex functional on $\mathbf{V}^{h}$.

3. Any $\mathbf{u}^{h}$ that minimizes $\mathscr{J}_{\gamma}(\cdot)$ on $\mathbf{V}^{h}$ is solution to Problem (16), that can be written equivalently

$$
\left\langle D \mathscr{J}_{\gamma}\left(\mathbf{u}^{h}\right), \mathbf{v}^{h}\right\rangle=0, \quad \forall \mathbf{v}^{h} \in \mathbf{V}^{h} .
$$

Proof. First, provided a large enough $\gamma_{0},\left(\mathscr{J}_{\gamma}\left(\mathbf{v}^{h}\right)+L\left(\mathbf{v}^{h}\right)\right)$ is a non-negative quantity due to the ellipticity of the elasticity tensor A, the discrete trace inequality (20) and the relationship $\left|\mathbf{P}_{1, \gamma}^{\mathbf{t}}\left(\mathbf{v}^{h}\right)-\left[\mathbf{P}_{1, \gamma}^{\mathbf{t}}\left(\mathbf{v}^{h}\right)\right]_{s}\right| \leq\left|\mathbf{P}_{1, \gamma}^{\mathbf{t}}\left(\mathbf{v}^{h}\right)\right|$, this latter being a property of the projection onto a closed ball.

Let us rewrite in a slightly different form the potential $\mathscr{J}_{\gamma}(\cdot)$ :

$$
\mathscr{J}_{\gamma}:=\tilde{\mathscr{J}}_{\mathrm{E}}+\tilde{\mathscr{J}}_{\gamma}^{\mathbf{n}}+\tilde{\mathscr{J}}_{\gamma}^{\mathbf{t}}
$$

with, for $\mathbf{v}^{h} \in \mathbf{V}^{h}$ :

$$
\begin{aligned}
\tilde{\mathscr{J}}_{\mathrm{E}}\left(\mathbf{v}^{h}\right) & :=\frac{1}{2} A_{\gamma}\left(\mathbf{v}^{h}, \mathbf{v}^{h}\right)-L\left(\mathbf{v}^{h}\right), \\
\tilde{J}_{\gamma} \mathbf{n}\left(\mathbf{v}^{h}\right) & :=\mathscr{J}_{\gamma}^{\mathbf{n}}\left(\mathbf{v}^{h}\right)+\frac{1}{2} \int_{\Gamma_{C}} \frac{1}{\gamma} \sigma_{n}\left(\mathbf{v}^{h}\right)^{2} d \Gamma, \\
\tilde{J}_{\gamma}^{\mathbf{t}}\left(\mathbf{v}^{h}\right) & :=\mathscr{J}_{\gamma}^{\mathbf{t}}\left(\mathbf{v}^{h}\right)+\frac{1}{2} \int_{\Gamma_{C}} \frac{1}{\gamma}\left|\sigma_{\mathbf{t}}\left(\mathbf{v}^{h}\right)\right|^{2} d \Gamma .
\end{aligned}
$$


The potential $\tilde{\mathscr{J}}_{\mathrm{E}}(\cdot)$ is Gâteaux-differentiable on $\mathbf{V}^{h}$ and its derivative is:

$$
\left\langle D \tilde{\mathscr{J}}_{\mathrm{E}}\left(\mathbf{v}^{h}\right), \mathbf{w}^{h}\right\rangle=A_{\gamma}\left(\mathbf{v}^{h}, \mathbf{w}^{h}\right)-L\left(\mathbf{w}^{h}\right),
$$

for all $\mathbf{v}^{h}, \mathbf{w}^{h} \in \mathbf{V}^{h}$.

Similarly we check that the potential $\tilde{\mathscr{J}}_{\gamma}^{\mathbf{n}}(\cdot)$ is Gâteaux-differentiable too on $\mathbf{V}^{h}$. Its derivative is obtained as

$$
\begin{aligned}
\left\langle D \tilde{\mathscr{J}} \mathbf{n}^{\mathbf{n}}\left(\mathbf{v}^{h}\right), \mathbf{w}^{h}\right\rangle & =\int_{\Gamma_{C}} \frac{1}{\gamma}\left[\mathrm{P}_{1, \gamma}^{\mathbf{n}}\left(\mathbf{v}^{h}\right)\right]_{\mathbb{R}^{-}}\left\langle D\left(\left[\mathrm{P}_{1, \gamma}^{\mathbf{n}}\left(\mathbf{v}^{h}\right)\right]_{\mathbb{R}^{-}}\right), \mathbf{w}^{h}\right\rangle d \Gamma \\
& =\int_{\Gamma_{C}} \frac{1}{\gamma}\left[\mathrm{P}_{1, \gamma}^{\mathbf{n}}\left(\mathbf{v}^{h}\right)\right]_{\mathbb{R}^{-}} H\left(-\mathrm{P}_{1, \gamma}^{\mathbf{n}}\left(\mathbf{v}^{h}\right)\right) \mathrm{P}_{1, \gamma}^{\mathbf{n}}\left(\mathbf{w}^{h}\right) d \Gamma \\
& =\int_{\Gamma_{C}} \frac{1}{\gamma}\left[\mathrm{P}_{1, \gamma}^{\mathbf{n}}\left(\mathbf{v}^{h}\right)\right]_{\mathbb{R}^{-}} \mathrm{P}_{1, \gamma}^{\mathbf{n}}\left(\mathbf{w}^{h}\right) d \Gamma
\end{aligned}
$$

where we used the property (9).

For the last potential $\tilde{J}_{\gamma}^{\mathbf{t}}(\cdot)$ let us consider the functional

$$
J: \mathbb{R}^{d-1} \ni \mathbf{x} \mapsto \frac{1}{2}\left|\mathbf{x}-[\mathbf{x}]_{s}\right|^{2} \in \mathbb{R} .
$$

After simple calculations we check that $J(\cdot)$ is Gâteaux-differentiable and that:

$$
\langle D J(\mathbf{x}), \mathbf{y}\rangle=\left(\mathbf{x}-[\mathbf{x}]_{s}\right) \cdot \mathbf{y},
$$

for all $\mathbf{x}, \mathbf{y} \in \mathbb{R}^{d-1}$. Using the above formula we obtain that $\tilde{J}_{\gamma}^{\mathbf{t}}(\cdot)$ is Gâteaux-differentiable, with derivative

$$
\left\langle D \tilde{\mathscr{J}}_{\gamma}^{\mathbf{t}}\left(\mathbf{v}^{h}\right), \mathbf{w}^{h}\right\rangle=-\int_{\Gamma_{C}} \frac{1}{\gamma}\left(\mathbf{P}_{1, \gamma}^{\mathbf{t}}\left(\mathbf{v}^{h}\right)-\left[\mathbf{P}_{1, \gamma}^{\mathbf{t}}\left(\mathbf{v}^{h}\right)\right]_{s}\right) \cdot \mathbf{P}_{1, \gamma}^{\mathbf{t}}\left(\mathbf{w}^{h}\right) d \Gamma+\int_{\Gamma_{C}} \frac{1}{\gamma} \mathbf{P}_{1, \gamma}^{\mathbf{t}}\left(\mathbf{v}^{h}\right) \cdot \mathbf{P}_{1, \gamma}^{\mathbf{t}}\left(\mathbf{w}^{h}\right) d \Gamma .
$$

This simplifies further into:

$$
\left\langle D \tilde{J}_{\gamma}^{\mathbf{t}}\left(\mathbf{v}^{h}\right), \mathbf{w}^{h}\right\rangle=\int_{\Gamma_{C}} \frac{1}{\gamma}\left[\mathbf{P}_{1, \gamma}^{\mathbf{t}}\left(\mathbf{v}^{h}\right)\right]_{s} \cdot \mathbf{P}_{1, \gamma}^{\mathbf{t}}\left(\mathbf{w}^{h}\right) d \Gamma
$$

The convexity of $\tilde{\mathcal{J}}_{\mathrm{E}}(\cdot)$ (resp. $\tilde{\mathcal{J}}_{\gamma}^{\mathbf{n}}(\cdot)$ and $\left.\tilde{\mathscr{J}}_{\gamma}^{\mathbf{t}}(\cdot)\right)$ results from the ellipticity of $\mathbf{A}$ and the inequality (20) (resp. inequalities (10) and (11)) combined with the characterization of Gâteaux-differentiable convex functions that can be found, in, e.g., [64, Theorem 3.3, Chapter 3]. This ends the proof of the second point in the theorem. To prove the last Point 3, we apply, e.g., [64, Theorem 3.7, (v), Chapter 3], with the expression of $D \mathscr{J}_{\gamma}(\cdot)$ that is the sum of the expressions (21)-(22)-(23).

Remark 10. Note that a same result can be obtained for the two-body contact problem discretized with an unbiased Nitsche method, see [29] for details.

\subsection{Relationship with other methods}

We discuss the relationship with other methods here: Nitsche for Dirichlet boundary conditions, Barbosa and Hughes stabilized method and the augmented lagrangian of Alart and Curnier. 


\subsubsection{Nitsche for contact and Nitsche for Dirichlet}

We consider the case of frictionless contact $(s=0)$ and Nitsche's formulation (17) in this situation. Let us split the contact boundary $\Gamma_{C}$ into two portions:

- $\Gamma_{C}^{-}:=\left\{\mathbf{x} \in \Gamma_{C}: \mathrm{P}_{1, \gamma}^{\mathbf{n}}\left(\mathbf{u}^{h}\right)(\mathbf{x})<0\right\}$,

- $\Gamma_{C}^{+}:=\left\{\mathbf{x} \in \Gamma_{C}: \mathrm{P}_{1, \gamma}^{\mathbf{n}}\left(\mathbf{u}^{h}\right)(\mathbf{x}) \geq 0\right\}\left(=\Gamma_{C} \backslash \Gamma_{C}^{-}\right)$.

In this case, we can rewrite formally the Nitsche-based method (17):

$$
\left\{\begin{array}{l}
\text { Find } \mathbf{u}^{h} \in \mathbf{V}^{h} \text { such that: } \\
A_{\theta \gamma}^{\mathbf{n}}\left(\mathbf{u}^{h}, \mathbf{v}^{h}\right)+\int_{\Gamma_{C}^{-}} \frac{1}{\gamma} \mathrm{P}_{1, \gamma}^{\mathbf{n}}\left(\mathbf{u}^{h}\right) \mathbf{P}_{\theta, \gamma}^{\mathbf{n}}\left(\mathbf{v}^{h}\right) d \Gamma=L\left(\mathbf{v}^{h}\right), \quad \forall \mathbf{v}^{h} \in \mathbf{V}^{h} .
\end{array}\right.
$$

Note that this only a formal writing since in fact the splitting of $\Gamma_{C}$ into $\Gamma_{C}^{+}$and $\Gamma_{C}^{-}$is an unknown, that depends on $\mathbf{u}^{h}$. Then using the detailed expression of $A_{\theta \gamma}^{\mathbf{n}}(\cdot, \cdot)$ and $\mathrm{P}_{1, \gamma}^{\mathbf{n}}(\cdot)$, and after re-ordering of the terms we get:

$$
\begin{aligned}
& a\left(\mathbf{u}^{h}, \mathbf{v}^{h}\right)-\int_{\Gamma_{C}^{+}} \frac{\theta}{\gamma} \sigma_{n}\left(\mathbf{u}^{h}\right) \sigma_{n}\left(\mathbf{v}^{h}\right) \\
& -\int_{\Gamma_{C}^{-}} \sigma_{n}\left(\mathbf{u}^{h}\right) v_{n}^{h} d \Gamma-\theta \int_{\Gamma_{C}^{-}} u_{n}^{h} \sigma_{n}\left(\mathbf{v}^{h}\right) d \Gamma+\int_{\Gamma_{C}^{-}} \gamma u_{n}^{h} v_{n}^{h} d \Gamma=L\left(\mathbf{v}^{h}\right), \quad \forall \mathbf{v}^{h} \in \mathbf{V}^{h} .
\end{aligned}
$$

We recognize on $\Gamma_{C}^{-}$Nitsche's method for imposition of Dirichlet boundary conditions on the normal component of the displacement $u_{n}$ (see, e.g., $\left.[75,85]\right)$. It results that $\Gamma_{C}^{-}$can be viewed as a discrete approximation of the actual contact surface. On $\Gamma_{C}^{+}$a free Neumann boundary condition is imposed weakly, in the same fashion as in $[62,73]$. Therefore $\Gamma_{C}^{+}$may represent a discrete approximation of the unsticked contact surface.

\subsubsection{Link with Barbosa \& Hughes stabilization}

We still consider the case of frictionless contact $(s=0)$ and Nitsche's formulation (17). Let us introduce

$$
L_{-}^{2}\left(\Gamma_{C}\right):=\left\{\mu \in L^{2}\left(\Gamma_{C}\right) \mid \mu \leq 0 \text { a.e. on } \Gamma_{C}\right\}
$$

and the discrete multiplier

$$
\lambda^{h}:=\left[\mathrm{P}_{1, \gamma}^{\mathbf{n}}\left(\mathbf{u}^{h}\right)\right]_{\mathbb{R}^{-}} .
$$

Following the same steps as in the symmetric case $\theta=1$ (see [23, Section 2.3] for details as well as [28] when $\theta \in \mathbb{R}$ ), we can rewrite the formulation (17) into an equivalent mixed form:

$$
\left\{\begin{array}{l}
\text { Find }\left(\mathbf{u}^{h}, \lambda^{h}\right) \in \mathbf{V}^{h} \times L_{-}^{2}\left(\Gamma_{C}\right) \text { such that: } \\
a\left(\mathbf{u}^{h}, \mathbf{v}^{h}\right)-\int_{\Gamma_{C}} \lambda^{h} v_{n}^{h} d \Gamma+\int_{\Gamma_{C}} \theta \gamma^{-1}\left(\lambda^{h}-\sigma_{n}\left(\mathbf{u}^{h}\right)\right) \sigma_{n}\left(\mathbf{v}^{h}\right) d \Gamma=L\left(\mathbf{v}^{h}\right), \quad \forall \mathbf{v}^{h} \in \mathbf{V}^{h}, \\
\int_{\Gamma_{C}}\left(\mu-\lambda^{h}\right) u_{n}^{h} d \Gamma+\int_{\Gamma_{C}} \gamma^{-1}\left(\mu-\lambda^{h}\right)\left(\lambda^{h}-\sigma_{n}\left(\mathbf{u}^{h}\right)\right) d \Gamma \geq 0, \quad \forall \mu \in L_{-}^{2}\left(\Gamma_{C}\right) .
\end{array}\right.
$$

The first line is simply expression (17) recasted after introduction of the multiplier $\lambda^{h}$, and the second line means that $\lambda^{h}$ is the projection of $\mathrm{P}_{1, \gamma}^{\mathbf{n}}\left(\mathbf{u}^{h}\right)$ onto $L_{-}^{2}\left(\Gamma_{C}\right)$. Note as well that the inverse of Nitsche parameter $\gamma_{0}^{-1}$ can be 
interpreted as a stabilization parameter. We recover indeed a mixed form close to the stabilized method [58], the only difference being that in [58], the dual set $L_{-}^{2}\left(\Gamma_{C}\right)$ is approximated by using finite elements on the contact boundary. The stabilized method of [58] is an adaptation to unilateral contact of Barbosa \& Hughes stabilization [7].

The method of stabilized Lagrange multiplier at the boundary proposed by Barbosa \& Hughes [7] originates from a stream of works dedicated to the use of a penalization technique for recovering coercivity for the Lagrange multiplier in order to avoid handling the Babuska-Brezzi condition in the finite element context. At the beginning the proposed formulation was inconsistent [76], then supplementary terms were added for ensuring consistency [7]. This method of stabilized Lagrange multiplier has been adapted for the unilateral contact problem in the frictionless case [58]. Optimal error estimates for the Lagrange multiplier have been obtained provided an extra regularity result for the Lagrange multiplier is satisfied, which in certain circumstances is not relevant, see remark 3.7 in [58] (note that using the new results published in [35] this analysis can be improved now).

In the seminal paper [85] a simplified formulation of Barbosa \& Hughes (where just the essential added terms are considered) has been proved equivalent to a Nitsche formulation for a Laplace problem with Dirichlet boundary conditions (for domain decomposition, see also the recent paper of Juntunen [61]). In this context the Lagrange multiplier belongs to $L^{2}\left(\Gamma_{C}\right)$ and is approximated with discontinuous finite elements. Therefore this Lagrange multiplier can be eliminated and a Nitsche formulation is recovered.

\subsubsection{Proximal augmented lagrangian and Nitsche}

A popular formulation for solving contact problems is the augmented lagrangian (see, e.g., $[2,81,82])$. In the case of unilateral contact problem without friction, its expression is:

$$
\mathscr{L}_{r}\left(\mathbf{u}^{h}, \lambda^{H}\right):=\frac{1}{2} a\left(\mathbf{u}^{h}, \mathbf{u}^{h}\right)-L\left(\mathbf{u}^{h}\right)+\int_{\Gamma_{C}} \frac{1}{2 r}\left(\left[\lambda^{H}-r u_{n}^{h}\right]_{\mathbb{R}^{-}}^{2}-\left(\lambda^{H}\right)^{2}\right) d \Gamma
$$

for $\mathbf{u}^{h} \in \mathbf{V}^{h}$ and where the discrete multiplier $\lambda^{H}$ belongs to a finite element space $W^{H}$ of functions defined on the contact boundary $\Gamma_{C}$. The multiplier $\lambda^{H}$ is a new unknown that approximates the normal stress $\sigma_{n}(\mathbf{u})$. We introduced as well $r>0$, which is the augmentation parameter. We note that

$$
\frac{d}{d x}\left(\frac{1}{2}[x]_{\mathbb{R}^{-}}^{2}\right)=[x]_{\mathbb{R}^{-}} .
$$

Therefore, using the notation $\left\langle\frac{\partial}{\partial \mathbf{u}}(\cdot), \cdot\right\rangle\left(\operatorname{resp} \cdot\left\langle\frac{\partial}{\partial \lambda}(\cdot), \cdot\right\rangle\right)$ for the directional derivative according to the first variable $\mathbf{u}$ (resp. to the second variable $\lambda$ ):

$$
\begin{aligned}
& \left\langle\frac{\partial}{\partial \mathbf{u}}\left(\int_{\Gamma_{C}} \frac{1}{2 r}\left[\lambda^{H}-r u_{n}^{h}\right]_{\mathbb{R}^{-}}^{2} d \Gamma\right), \mathbf{v}^{h}\right\rangle \\
= & \int_{\Gamma_{C}} \frac{1}{r}\left[\lambda^{H}-r u_{n}^{h}\right]_{\mathbb{R}^{-}}\left(-r v_{n}^{h}\right) d \Gamma=-\int_{\Gamma_{C}}\left[\lambda^{H}-r u_{n}^{h}\right]_{\mathbb{R}^{-}} v_{n}^{h} d \Gamma .
\end{aligned}
$$

Similarly

$$
\left\langle\frac{\partial}{\partial \lambda}\left(\int_{\Gamma_{C}} \frac{1}{2 r}\left[\lambda^{H}-r u_{n}^{h}\right]_{\mathbb{R}^{-}}^{2} d \Gamma\right), \mu^{H}\right\rangle=\int_{\Gamma_{C}} \frac{1}{r}\left[\lambda^{H}-r u_{n}^{h}\right]_{\mathbb{R}^{-}} \mu^{H} d \Gamma .
$$


Using the above expressions, let us write explicitly the optimality system associated to the augmented lagrangian (24):

$$
\begin{aligned}
& 0=\left\langle\frac{\partial \mathscr{L}_{r}}{\partial \mathbf{u}}\left(\mathbf{u}^{h}, \lambda^{H}\right), \mathbf{v}^{H}\right\rangle=a\left(\mathbf{u}^{h}, \mathbf{v}^{h}\right)-L\left(\mathbf{v}^{h}\right)-\int_{\Gamma_{C}}\left[\lambda^{H}-r u_{n}^{h}\right]_{\mathbb{R}^{-}} v_{n}^{h} d \Gamma, \quad \forall \mathbf{v}^{h} \in \mathbf{V}^{h}, \\
& 0=\left\langle\frac{\partial \mathscr{L}_{r}}{\partial \lambda}\left(\mathbf{u}^{h}, \lambda^{H}\right), \mu^{H}\right\rangle=\int_{\Gamma_{C}} \frac{1}{r}\left(\left[\lambda^{H}-r u_{n}^{h}\right]_{\mathbb{R}^{-}}-\lambda^{H}\right) \mu^{H} d \Gamma, \quad \forall \mu^{H} \in W^{H} .
\end{aligned}
$$

This is an unconstrained formulation, that is more appropriate for numerical solving. Now, remark that the second equation is a way to enforce weakly, at the discrete level, the condition (12). Another way, straightforward, to enforce this condition, is to substitute $\sigma_{n}\left(\mathbf{u}^{h}\right)$ to $\lambda^{H}$ in the first equation of the optimality system (and we forget about the second equation):

$$
a\left(\mathbf{u}^{h}, \mathbf{v}^{h}\right)-\int_{\Gamma_{C}}\left[\sigma_{n}\left(\mathbf{u}^{h}\right)-r u_{n}^{h}\right]_{\mathbb{R}^{-}} v_{n}^{h} d \Gamma=L\left(\mathbf{v}^{h}\right), \quad \forall \mathbf{v}^{h} \in \mathbf{V}^{h} .
$$

We now recognize Nitsche's method (17) for $\theta=0$. Moreover the parameter $\gamma$ can be identified with the augmentation parameter $r$. Note that some recent works are filling the gap between Nitsche and augmented lagrangian formulations in the case of contact and obstacle problems [17, 19, 48].

\section{Analysis of the Nitsche-based method}

This section sums up the main results about the numerical analysis of the Nitsche-based formulation (16). For detailed proofs, the reader is refered to [23, 28, 21, 22]. First of all we recall the consistency of the method, that is a direct consequence of reformulations (12)-(13) followed by integration-by-parts:

Lemma 2. The Nitsche-based method (16) is consistent: suppose that the solution $\mathbf{u}$ to (1)-(3) belongs to $\left(H^{\frac{3}{2}+v}(\Omega)\right)^{d}$, with $v>0$, then $\mathbf{u}$ is also solution to

$$
A_{\theta \gamma}\left(\mathbf{u}, \mathbf{v}^{h}\right)+\int_{\Gamma_{C}} \frac{1}{\gamma}\left[\mathrm{P}_{1, \gamma}^{\mathbf{n}}(\mathbf{u})\right]_{\mathbb{R}^{-}} \mathbf{P}_{\theta, \gamma}^{\mathbf{n}}\left(\mathbf{v}^{h}\right) d \Gamma+\int_{\Gamma_{C}} \frac{1}{\gamma}\left[\mathbf{P}_{1, \gamma}^{\mathbf{t}}(\mathbf{u})\right]_{s} \cdot \mathbf{P}_{\theta, \gamma}^{\mathbf{t}}\left(\mathbf{v}^{h}\right) d \Gamma=L\left(\mathbf{v}^{h}\right),
$$

for any $\mathbf{v}^{h} \in \mathbf{V}^{h}$.

Note that for the same reasons the formulation (17) (resp. (18)) for frictionless unilateral contact (resp. for frictional bilateral contact) is consistent too.

\subsection{Well-posedness}

To show that Problem (16) is well-posed we use an argument by Brezis for M-type and pseudo-monotone operators [13] (see also [71] and [65]). We define a (non-linear) operator $\mathbf{B}_{s}^{h}: \mathbf{V}^{h} \rightarrow \mathbf{V}^{h}$, by using the Riesz representation theorem and by means of the formula:

$$
\left(\mathbf{B}_{s}^{h} \mathbf{v}^{h}, \mathbf{w}^{h}\right)_{\gamma}:=A_{\theta \gamma}\left(\mathbf{v}^{h}, \mathbf{w}^{h}\right)+\int_{\Gamma_{C}} \frac{1}{\gamma}\left[\mathrm{P}_{1, \gamma}^{\mathbf{n}}\left(\mathbf{v}^{h}\right)\right]_{\mathbb{R}^{-}} \mathrm{P}_{\theta, \gamma}^{\mathbf{n}}\left(\mathbf{w}^{h}\right) d \Gamma+\int_{\Gamma_{C}} \frac{1}{\gamma}\left[\mathbf{P}_{1, \gamma}^{\mathbf{t}}\left(\mathbf{v}^{h}\right)\right]_{s} \cdot \mathbf{P}_{\theta, \gamma}^{\mathbf{t}}\left(\mathbf{w}^{h}\right) d \Gamma
$$


Note that Problem (16) is well-posed if and only if $\mathbf{B}_{s}^{h}$ is one-to-one. The following result characterizes wellposedness:

Theorem 1. The operator $\mathbf{B}_{s}^{h}$ is hemicontinuous. Moreover there exist $C, C^{\prime}>0$ such that, for all $\mathbf{v}^{h}, \mathbf{w}^{h} \in \mathbf{V}^{h}$ :

$$
\left(\mathbf{B}_{s}^{h} \mathbf{v}^{h}-\mathbf{B}_{s}^{h} \mathbf{w}^{h}, \mathbf{v}^{h}-\mathbf{w}^{h}\right)_{\gamma} \geq C^{\prime}\left(1-\frac{C(1+\theta)^{2}}{2 \gamma_{0}}\right)\left\|\mathbf{v}^{h}-\mathbf{w}^{h}\right\|_{\gamma}^{2}
$$

As a result, when the condition below holds

$$
\gamma_{0} \geq C(1+\theta)^{2}
$$

Problem (16) admits one unique solution $\mathbf{u}^{h}$ in $\mathbf{V}^{h}$.

Remark 11. In the symmetric case $\theta=1$, we remark that:

$$
\left\langle D \mathscr{J}_{\gamma}\left(\mathbf{v}^{h}\right), \mathbf{w}^{h}\right\rangle=\left(\mathbf{B}_{s}^{h} \mathbf{v}^{h}, \mathbf{w}^{h}\right)_{\gamma}-L\left(\mathbf{w}^{h}\right),
$$

for $\mathbf{v}^{h}, \mathbf{w}^{h} \in \mathbf{V}^{h}$, in other terms $\mathbf{B}_{s}^{h}$ is the gradient of $\left(\mathscr{J}_{\gamma}+L\right)(\cdot)$. In this case the equation (27) means that $\mathscr{J}_{\gamma}(\cdot)$ is strongly convex under the condition (28). As a result, when $\theta=1$, well-posedness can alternatively be established using a minimization argument, such as [64, Theorem 3.4, Chapter 3], and the unique solution to (16) is also the unique minimizer of $\mathscr{J}_{\gamma}(\cdot)$ on $\mathbf{V}^{h}$.

\subsection{A priori error estimates in $H^{1}(\Omega)$-norm}

First we recall the abstract error estimate.

Theorem 2. Suppose that the solution $\mathbf{u}$ to Problem (6) belongs to $\left(H^{\frac{3}{2}+v}(\Omega)\right)^{d}$ with $v>0$ and $d=2$ or $d=3$. 1. Let $\theta \in \mathbb{R}$. Suppose that the parameter $\gamma_{0}>0$ is sufficiently large. Then the solution $\mathbf{u}^{h}$ to Problem (16) satisfies the following abstract error estimate:

$$
\begin{aligned}
& \left\|\mathbf{u}-\mathbf{u}^{h}\right\|_{1, \Omega}+\gamma_{0}^{-\frac{1}{2}}\left(\left\|\sigma_{n}(\mathbf{u})-\left[\mathrm{P}_{1, \gamma}^{\mathbf{n}}\left(\mathbf{u}^{h}\right)\right]_{\mathbb{R}^{-}}\right\|_{-\frac{1}{2}, h, \Gamma_{C}}+\left\|\sigma_{\mathbf{t}}(\mathbf{u})-\left[\mathbf{P}_{1, \gamma}^{\mathbf{t}}\left(\mathbf{u}^{h}\right)\right]_{s}\right\|_{-\frac{1}{2}, h, \Gamma_{C}}\right) \\
\leq & C \inf _{\mathbf{v}^{h} \in \mathbf{V}^{h}}\left(\left\|\mathbf{u}-\mathbf{v}^{h}\right\|_{1, \Omega}+\gamma_{0}^{\frac{1}{2}}\left\|\mathbf{u}-\mathbf{v}^{h}\right\|_{\frac{1}{2}, h, \Gamma_{C}}+\gamma_{0}^{-\frac{1}{2}}\left\|\sigma\left(\mathbf{u}-\mathbf{v}^{h}\right) \mathbf{n}\right\|_{-\frac{1}{2}, h, \Gamma_{C}}\right),
\end{aligned}
$$

where $C$ is a positive constant, independent of $h, \mathbf{u}$ and $\gamma_{0}$.

2. Set $\theta=-1$. Then for all values of $\gamma_{0}>0$, the solution $\mathbf{u}^{h}$ to Problem (16) satisfies the abstract error estimate (29) where $C$ is a positive constant, dependent of $\gamma_{0}$ but independent of $h$ and $\mathbf{u}$.

The optimal convergence of the method is stated below.

Theorem 3. Suppose that the solution $\mathbf{u}$ to Problem (6) belongs to $\left(H^{\frac{3}{2}+v}(\Omega)\right)^{d}$ with $0<v \leq k-\frac{1}{2}(k=1,2$ is the degree of the finite element method, given in (8)) and $d=2,3$. When $\theta \neq-1$, suppose in addition that the parameter $\gamma_{0}$ is sufficiently large. The solution $\mathbf{u}^{h}$ to Problem (16) satisfies the following error estimate:

$$
\left\|\mathbf{u}-\mathbf{u}^{h}\right\|_{1, \Omega}+\left\|\sigma_{n}(\mathbf{u})-\left[\mathrm{P}_{1, \gamma}^{\mathbf{n}}\left(\mathbf{u}^{h}\right)\right]_{\mathbb{R}^{-}}\right\|_{-\frac{1}{2}, h, \Gamma_{C}}+\left\|\sigma_{\mathbf{t}}(\mathbf{u})-\left[\mathbf{P}_{1, \gamma}^{\mathbf{t}}\left(\mathbf{u}^{h}\right)\right]_{s}\right\|_{-\frac{1}{2}, h, \Gamma_{C}} \leq C h^{\frac{1}{2}+v}\|\mathbf{u}\|_{\frac{3}{2}+v, \Omega},
$$


where $C$ is a positive constant, independent of $h$ and $\mathbf{u}$.

We can easily obtain the following error estimate on the Cauchy constraint $\left\|\sigma\left(\mathbf{u}-\mathbf{u}^{h}\right) \mathbf{n}\right\|_{-\frac{1}{2}, h, \Gamma_{C}}$ in the weighted $L^{2}\left(\Gamma_{C}\right)$-norm (note that $\sigma_{n}\left(\mathbf{u}^{h}\right) \neq\left[\mathrm{P}_{1, \gamma}^{\mathbf{n}}\left(\mathbf{u}^{h}\right)\right]_{\mathbb{R}^{-}}$and $\sigma_{\mathbf{t}}\left(\mathbf{u}^{h}\right) \neq\left[\mathbf{P}_{1, \gamma}^{\mathbf{t}}\left(\mathbf{u}^{h}\right)\right]_{S}$ on $\Gamma_{C}$ conversely to the continuous case).

Corollary 1. Suppose that the solution $\mathbf{u}$ to Problem (6) belongs to $\left(H^{\frac{3}{2}+v}(\Omega)\right)^{d}$ with $0<v \leq k-\frac{1}{2}$ and $d=2,3$. When $\theta \neq-1$, suppose in addition that the parameter $\gamma_{0}$ is sufficiently large. The solution $\mathbf{u}^{h}$ to Problem (16) satisfies the following error estimate:

$$
\left\|\sigma\left(\mathbf{u}-\mathbf{u}^{h}\right) \mathbf{n}\right\|_{-\frac{1}{2}, h, \Gamma_{C}} \leq C h^{\frac{1}{2}+v}\|\mathbf{u}\|_{\frac{3}{2}+v, \Omega},
$$

where $C$ is a positive constant, independent of $h$ and $\mathbf{u}$.

\subsection{Residual-based a posteriori error estimate}

An explicit residual-based a posteriori error estimate can be derived for Problem (16), that is an extension of the one presented in [8] (see also, e.g., [87] for linear elasticity). We introduce standard notations for this purpose:

- We define $E_{h}$ the set of edges/faces of the triangulation and define $E_{h}^{\text {int }}:=\left\{E \in E_{h}: E \subset \Omega\right\}$ as the set of interior edges/faces of $\mathscr{T}^{h}$. We denote by $E_{h}^{N}:=\left\{E \in E_{h}: E \subset \Gamma_{N}\right\}$ the set of Neumann edges/faces and similarly $E_{h}^{C}:=\left\{E \in E_{h}: E \subset \Gamma_{C}\right\}$ is the set of contact edges/faces.

- For an element $T$, we denote by $E_{T}$ the set of edges/faces of $T$ and according to the above notation, we set $E_{T}^{i n t}:=E_{T} \cap E_{h}^{i n t}, E_{T}^{N}:=E_{T} \cap E_{h}^{N}, E_{T}^{C}:=E_{T} \cap E_{h}^{C}$.

- For an edge/face $E$ of an element $T$, introduce $v_{T, E}$ the unit outward normal vector to $T$ along $E$. Furthermore, for each edge/face $E$, we fix one of the two normal vectors and denote it by $v_{E}$. The jump of some vector valued function $\mathbf{v}$ across an edge/face $E \in E_{h}^{\text {int }}$ at a point $\mathbf{y} \in E$ is defined as

$$
\llbracket \mathbf{v} \rrbracket_{E}(\mathbf{y}):=\lim _{\alpha \rightarrow 0^{+}} \mathbf{v}\left(\mathbf{y}+\alpha v_{E}\right)-\mathbf{v}\left(\mathbf{y}-\alpha v_{E}\right)
$$

- Let $\omega_{T}$ be the union of all elements having a nonempty intersection with $T$. Similarly for a node $x$ and an edge/face $E$, let $\omega_{x}:=\cup_{T: x \in T} T$ and $\omega_{E}:=\cup_{x \in \bar{E}} \omega_{x}$.

- $\mathbf{f}_{T}$ (resp. $\mathbf{f}_{N, E}$ ) is a computable quantity that approximates $\mathbf{f}$ on the element $T \in \mathscr{T}^{h}$ (resp. $\mathbf{f}_{N}$ on the edge $\left.E \in E_{h}^{N}\right)$.

The a posteriori error estimator is defined below.

Definition 3. The local error estimators $\eta_{T}$ and the the global estimator $\eta$ are defined by 


$$
\begin{aligned}
\eta_{T} & :=\left(\sum_{i=1}^{4} \eta_{i T}^{2}\right)^{1 / 2}, \\
\eta_{1 T} & :=h_{T}\left\|\operatorname{div} \sigma\left(\mathbf{u}^{h}\right)+\mathbf{f}_{T}\right\|_{0, T} \\
\eta_{2 T} & :=h_{T}^{1 / 2}\left(\sum_{E \in E_{T}^{i n t} \cup E_{T}^{N}}\left\|J_{E, n}\left(\mathbf{u}^{h}\right)\right\|_{0, E}^{2}\right)^{1 / 2}, \\
\eta_{3 T} & :=h_{T}^{1 / 2}\left(\sum_{E \in E_{T}^{C}}\left\|\sigma_{\mathbf{t}}\left(\mathbf{u}^{h}\right)-\left[\mathbf{P}_{1, \gamma}^{\mathbf{t}}\left(\mathbf{u}^{h}\right)\right]_{S}\right\|_{0, E}^{2}\right)^{1 / 2}, \\
\eta_{4 T} & :=h_{T}^{1 / 2}\left(\sum_{E \in E_{T}^{C}}\left\|\sigma_{n}\left(\mathbf{u}^{h}\right)-\left[\mathrm{P}_{1, \gamma}^{\mathbf{n}}\left(\mathbf{u}^{h}\right)\right]_{\mathbb{R}^{-}}\right\|_{0, E}^{2}\right)^{1 / 2}, \\
\eta & :=\left(\sum_{T \in \mathscr{T} h} \eta_{T}^{2}\right)^{1 / 2},
\end{aligned}
$$

where $J_{E, n}\left(\mathbf{u}^{h}\right)$ means the constraint jump of $\mathbf{u}^{h}$ in the normal direction, i.e.,

$$
J_{E, n}\left(\mathbf{u}^{h}\right):= \begin{cases}{\left[\left[\sigma\left(\mathbf{u}^{h}\right) v_{E}\right]_{E},\right.} & \forall E \in E_{h}^{i n t}, \\ \sigma\left(\mathbf{u}^{h}\right) v_{E}-\mathbf{f}_{N, E}, & \forall E \in E_{h}^{N}\end{cases}
$$

The local and global approximation terms are given by

$$
\begin{aligned}
& \zeta_{T}:=\left(h_{T}^{2} \sum_{T^{\prime} \subset \omega_{T}}\left\|\mathbf{f}-\mathbf{f}_{T^{\prime}}\right\|_{0, T^{\prime}}^{2}+h_{E} \sum_{E \subset E_{T}^{N}}\left\|\mathbf{f}_{N}-\mathbf{f}_{N, E}\right\|_{0, E}^{2}\right)^{1 / 2} \\
& \zeta:=\left(\sum_{T \in \mathscr{T} h} \zeta_{T}^{2}\right)^{1 / 2} .
\end{aligned}
$$

We need for the analysis a "saturation" assumption as in [8] for Nitsche-based domain decomposition, and as in [88] for mortar methods.

Assumption 4 The solution $\mathbf{u}$ to (1)-(2)-(3) and the discrete solution $\mathbf{u}^{h}$ to (16) are such that:

$$
\left\|\sigma_{n}\left(\mathbf{u}-\mathbf{u}^{h}\right)\right\|_{-1 / 2, h, \Gamma_{C}}+\left\|\sigma_{\mathbf{t}}\left(\mathbf{u}-\mathbf{u}^{h}\right)\right\|_{-1 / 2, h, \Gamma_{C}} \leq C\left\|\mathbf{u}-\mathbf{u}^{h}\right\|_{1, \Omega},
$$

where $C$ is a positive constant independent of $h$.

The following statement guarantees the reliability of the a posteriori error estimator:

Theorem 5. Let $\mathbf{u}$ be the solution to (1)-(2)-(3), with $\mathbf{u} \in\left(H^{\frac{3}{2}+v}(\Omega)\right)^{d}(v>0$ and $d=2,3)$, and let $\mathbf{u}^{h}$ be the solution to the corresponding discrete problem (16). Assume that, for $\theta \neq-1, \gamma_{0}$ is sufficiently large. Assume that the saturation assumption (33) holds as well. Then we have 


$$
\begin{gathered}
\left\|\mathbf{u}-\mathbf{u}^{h}\right\|_{1, \Omega}+\left\|\sigma_{n}(\mathbf{u})-\left[\mathrm{P}_{1, \gamma}^{\mathbf{n}}\left(\mathbf{u}^{h}\right)\right]_{\mathbb{R}^{-}}\right\|_{-\frac{1}{2}, h, \Gamma_{C}}+\left\|\sigma_{\mathbf{t}}(\mathbf{u})-\left[\mathbf{P}_{1, \gamma}^{\mathbf{t}}\left(\mathbf{u}^{h}\right)\right]_{s}\right\|_{-\frac{1}{2}, h, \Gamma_{C}} \\
+\left\|\sigma_{n}(\mathbf{u})-\sigma_{n}\left(\mathbf{u}^{h}\right)\right\|_{-1 / 2, h, \Gamma_{C}}+\left\|\sigma_{\mathbf{t}}(\mathbf{u})-\sigma_{\mathbf{t}}\left(\mathbf{u}^{h}\right)\right\|_{-1 / 2, h, \Gamma_{C}} \leq C\left(1+\gamma_{0}^{-1}\right)(\eta+\zeta),
\end{gathered}
$$

where the positive constant $C$ is independent of $h$ and $\gamma_{0}$.

The last result concerns the local lower error bounds of the discretization error terms:

Theorem 6. For all elements $T \in \mathscr{T}^{h}$, the following local lower error bounds hold:

$$
\begin{aligned}
& \eta_{1 T} \leq C\left\|\mathbf{u}-\mathbf{u}^{h}\right\|_{1, T}+\zeta_{T}, \\
& \eta_{2 T} \leq C\left\|\mathbf{u}-\mathbf{u}^{h}\right\|_{1, \omega_{T}}+\zeta_{T} .
\end{aligned}
$$

For all elements $T$ such that $T \cap E_{h}^{C} \neq \emptyset$, the following local lower error bounds hold:

$$
\begin{aligned}
& \eta_{3 T} \leq C \sum_{E \in E_{T}^{C}} h_{T}^{1 / 2}\left(\left\|\sigma_{\mathbf{t}}(\mathbf{u})-\left[\mathbf{P}_{1, \gamma}^{\mathbf{t}}\left(\mathbf{u}^{h}\right)\right]_{s}\right\|_{0, E}+\left\|\sigma_{\mathbf{t}}\left(\mathbf{u}-\mathbf{u}^{h}\right)\right\|_{0, E}\right) \\
& \eta_{4 T} \leq C \sum_{E \in E_{T}^{C}} h_{T}^{1 / 2}\left(\left\|\sigma_{n}(\mathbf{u})-\left[\mathrm{P}_{1, \gamma}^{\mathbf{n}}\left(\mathbf{u}^{h}\right)\right]_{\mathbb{R}^{-}}\right\|_{0, E}+\left\|\sigma_{n}\left(\mathbf{u}-\mathbf{u}^{h}\right)\right\|_{0, E}\right),
\end{aligned}
$$

where the positive constant $C$ is independent of $h$ and $\gamma_{0}$.

Remark 12. From Theorem 6, optimal convergence rates of order $O\left(h^{\min \left(k, \frac{1}{2}+v\right)}\right)$ are expected for the estimator of Definition 3.

\section{Numerical experiments}

This section is devoted to numerical results that illustrate the theoretical analysis and the practical interest of the method, in the frictionless case. First, in 4.1 we provide practical details concerning the implementation. Then in 4.2 we assess numerically the a priori error estimates of Subsection 3.2. Numerical assessment of the a posteriori error estimator described in 3.3 and adaptive computations are object of Subsection 4.3.

\subsection{Some implementation issues}

The discrete contact problem is solved by using a generalized Newton method, which means that Problem (16) is derived with respect to $\mathbf{u}^{h}$ to obtain the tangent system. The term "generalized Newton's method" comes from the fact that the operators such as $[\cdot]_{\mathbb{R}^{-}}$and $[\cdot]_{s}$ are not Gâteaux-differentiable at some specific points. However, no special treatment is considered. If a point of non-differentiability is encountered, the tangent system corresponding to one of the two alternatives ( $x<0$ or $x>0$ for $[\cdot]_{\mathbb{R}^{-}}$) is chosen arbitrarily. Integrals of the non-linear term on $\Gamma_{C}$ are computed with standard quadrature formulas. Note that, for frictionless contact, the situation where the solution is non-differentiable at an integration point is very rare and corresponds to what is called a "grazing contact" (both $u_{n}=0$ and $\sigma_{n}=0$ ). In [81] one can find further details and references on generalized Newton's method, and 
especially a numerical study of its convergence when applied to contact problems discretized by Nitsche's method (for variants $\theta=1$ and $\theta=0$ ) as well as other methods.

The finite element library Getfem $++{ }^{1}$ has been used for all the computations presented in this paper.

\subsection{Numerics for Hertz's contact}

The numerical results obtained in [28] for frictionless Hertz's contact problems of a disk/sphere with a plane rigid foundation are summarized here. This slightly exceeds the scope defined in Section 2 since a non-zero initial gap between the elastic solid and the rigid foundation is considered in the computations. Moreover, the tests are performed with $\mathbb{P}_{1}$ and isoparametric $\mathbb{P}_{2}$ Lagrange finite elements on meshes which are approximations of the real domain.

The numerical situation in two-dimensions is represented in Fig. 2. A disc of radius $20 \mathrm{~cm}$ is considered with a contact boundary $\Gamma_{C}$ which is restricted to the lower part $(y<20 \mathrm{~cm})$ of the boundary. A homogeneous Neumann condition is applied on the remaining part of the boundary. Since no Dirichlet condition is considered, the problem is not fully coercive. To overcome the non-definiteness coming from the free rigid motions, the horizontal displacement is prescribed to be zero on the two points of coordinates $(0 \mathrm{~cm}, 10 \mathrm{~cm})$ and $(0 \mathrm{~cm}, 30 \mathrm{~cm})$ which blocks the horizontal translation and the rigid rotation. Homogeneous isotropic linear elasticity in plane strain approximation is considered with a Young modulus fixed at $E=25 \mathrm{MPa}$ and a Poisson ratio $P=0.25$. A vertical density of volume forces of $20 \mathrm{MN} / \mathrm{m}^{3}$ is applied.
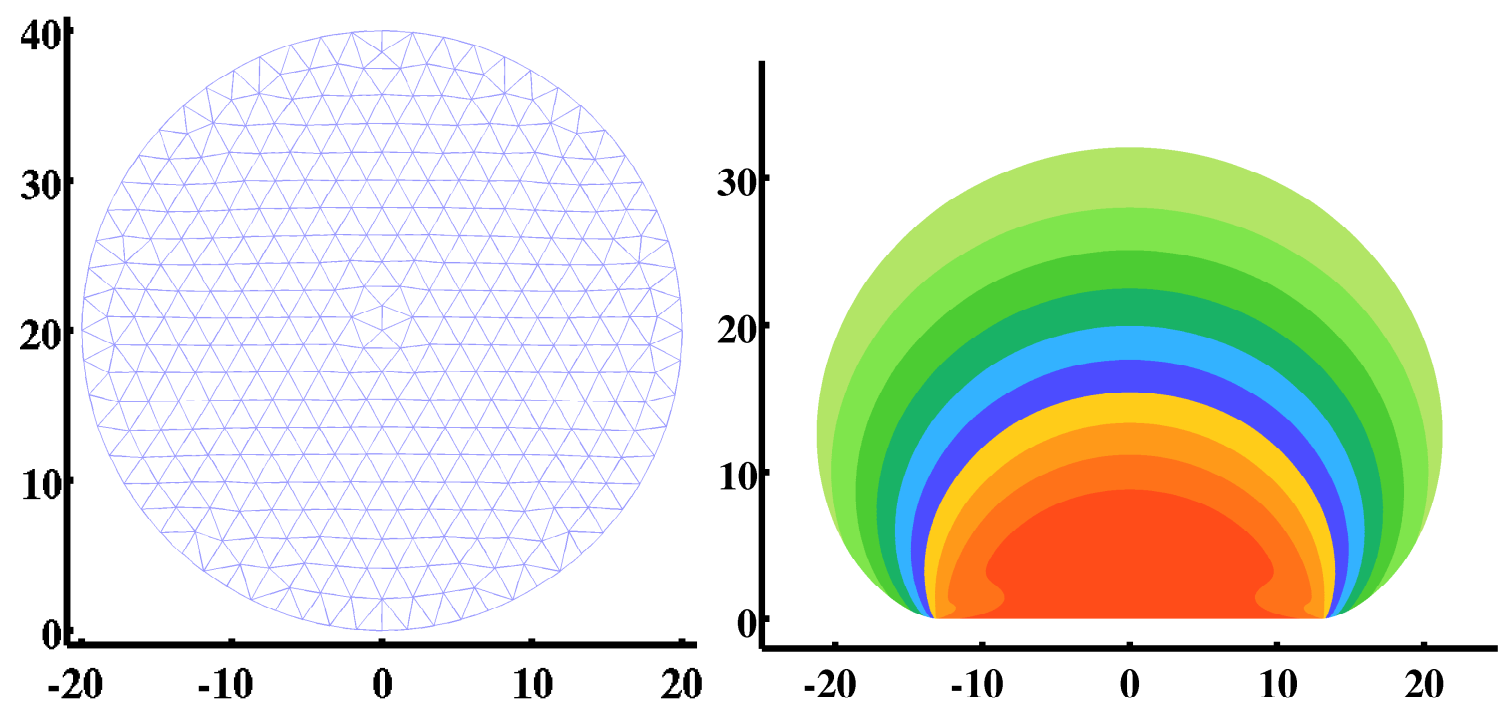

Fig. 2 Example of two-dimensional mesh and reference solution (with color plot of the Von-Mises stress).

The solution for mesh sizes $h=0.5 \mathrm{~cm}, 1 \mathrm{~cm}, 3 \mathrm{~cm}, 4.5 \mathrm{~cm}$ and $h=10 \mathrm{~cm}$ are compared with a reference solution on a very fine mesh $(h=0.15 \mathrm{~cm})$ using quadratic isoparametric finite elements. Moreover, the reference solution

${ }^{1}$ see www.getfem.org 
is computed with a different discretization of the contact problem (Lagrange multipliers and Alart-Curnier augmented lagrangian, see [81]). In complement to the relative error in $H^{1}(\Omega)$-norm we compute also the following relative error in $L^{2}\left(\Gamma_{C}\right)$-norm:

$$
\frac{\left\|\gamma^{-\frac{1}{2}}\left(\left[\mathrm{P}_{1, \gamma}^{\mathbf{n}}\left(\mathbf{u}^{h}\right)\right]_{\mathbb{R}^{-}}-\left[\mathrm{P}_{1, \gamma}^{\mathbf{n}}\left(\mathbf{u}_{r e f}^{h}\right)\right]_{\mathbb{R}^{-}}\right)\right\|_{0, \Gamma_{C}}}{\left\|\left[\mathrm{P}_{1, \gamma}^{\mathbf{n}}\left(\mathbf{u}_{r e f}^{h}\right)\right]_{\mathbb{R}^{-}}\right\|_{0, \Gamma_{C}}}
$$

where $\mathbf{u}^{h}$ is the discrete solution and $\mathbf{u}_{r e f}^{h}$ the reference solution. Note that $\left[\mathrm{P}_{1, \gamma}^{\mathbf{n}}\left(\mathbf{u}^{h}\right)\right]_{\mathbb{R}^{-}}$is an approximation of the contact stress with a convergence of order 1 (see Theorem 3 ). The convergence rates computed thanks to this test are reported in Table 1 and Table 2, for $\mathbb{P}_{1}$ and $\mathbb{P}_{2}$ finite elements, respectively, and for different values of $\theta$ and $\gamma_{0}$.

\begin{tabular}{c|lc}
$\gamma_{0} / E$ & $H^{1}(\Omega)$ & $L^{2}\left(\Gamma_{C}\right)$ \\
\hline 100 & 1.20 & 1.43 \\
1 & 0.84 & 0.61 \\
0.01 & 1.35 & 0.53 \\
& $\theta=1$
\end{tabular}

\begin{tabular}{c|ll}
$\gamma_{0} / E$ & $H^{1}(\Omega)$ & $L^{2}\left(\Gamma_{C}\right)$ \\
\hline 100 & 1.20 & 1.43 \\
1 & 1.23 & 1.35 \\
0.01 & 0.89 & 0.82 \\
& $\theta=0$
\end{tabular}

\begin{tabular}{c|cc}
$\gamma_{0} / E$ & $H^{1}(\Omega)$ & $L^{2}\left(\Gamma_{C}\right)$ \\
\hline 100 & 1.21 & 1.43 \\
1 & 1.32 & 1.32 \\
0.01 & 1.63 & 1.47 \\
\multicolumn{3}{|c}{$\theta=-1$}
\end{tabular}

Table 1 Computed convergence rates for Hertz's problem in $2 \mathrm{D}$ and $\mathbb{P}_{1}$ finite elements. The column " $H^{1}(\Omega)$ " stands for the relative error in $H^{1}(\Omega)$-norm on the displacement, the column " $L^{2}\left(\Gamma_{C}\right)$ " stands for the relative error in $L^{2}\left(\Gamma_{C}\right)$ on the contact condition.

\begin{tabular}{|c|c|c|c|c|c|c|c|c|}
\hline \multicolumn{3}{|c|}{$\gamma_{0} / E \mid H^{1}(\Omega) L^{2}\left(\Gamma_{C}\right)$} & \multicolumn{3}{|c|}{$\gamma_{0} / E \mid H^{1}(\Omega) L^{2}\left(\Gamma_{C}\right)$} & \multicolumn{3}{|c|}{$\gamma_{0} / E \mid H^{1}(\Omega) L^{2}\left(\Gamma_{C}\right)$} \\
\hline 100 & 1.62 & 1.45 & 100 & 1.63 & 1.42 & 100 & 1.64 & 1.42 \\
\hline 1 & 0.14 & 0.44 & 1 & 1.63 & 1.50 & 1 & 1.75 & 1.55 \\
\hline 0.01 & 1.00 & 0.58 & 0.01 & 1.43 & 1.24 & 0.01 & 1.94 & 1.61 \\
\hline \multicolumn{3}{|c|}{$\theta=1$} & \multicolumn{3}{|c|}{$\theta=0$} & \multicolumn{3}{|c|}{$\theta=-1$} \\
\hline
\end{tabular}

Table 2 Computed convergence rates for Hertz's problem in $2 \mathrm{D}$ and $\mathbb{P}_{2}$ finite elements. The column " $H^{1}(\Omega)$ " stands for the relative error in $H^{1}(\Omega)$-norm on the displacement, the column " $L^{2}\left(\Gamma_{C}\right)$ " stands for the relative error in $L^{2}\left(\Gamma_{C}\right)$ on the contact condition.

For the 3D Hertz's problem and $\mathbb{P}_{1}$ finite elements, convergence rates are reported in Table 3.

\begin{tabular}{|c|c|c|c|c|c|c|c|c|}
\hline \multicolumn{3}{|c|}{$\gamma_{0} / E \mid H^{1}(\Omega) L^{2}\left(\Gamma_{C}\right)$} & \multicolumn{3}{|c|}{$\gamma_{0} / E \mid H^{1}(\Omega) L^{2}\left(\Gamma_{C}\right)$} & \multicolumn{3}{|c|}{$\gamma_{0} / E \mid H^{1}(\Omega) L^{2}\left(\Gamma_{C}\right)$} \\
\hline 100 & 1.62 & 1.12 & $\overline{100}$ & 1.59 & 1.10 & 100 & 1.56 & 1.07 \\
\hline 1 & -0.21 & 0.81 & 1 & 1.00 & 1.94 & 1 & 1.44 & 1.85 \\
\hline 0.01 & -0.47 & 0.51 & 0.01 & 0.40 & 1.25 & 0.01 & 1.42 & 1.41 \\
\hline \multicolumn{3}{|c|}{$\theta=1$} & \multicolumn{3}{|c|}{$\theta=0$} & \multicolumn{3}{|c|}{$\theta=-1$} \\
\hline
\end{tabular}

Table 3 Computed convergence rates for Hertz's problem in $3 \mathrm{D}$ and $\mathbb{P}_{1}$ finite elements. The column " $H^{1}(\Omega)$ " stands for the relative error in $H^{1}(\Omega)$-norm on the displacement, the column " $L^{2}\left(\Gamma_{C}\right)$ " stands for the relative error in $L^{2}\left(\Gamma_{C}\right)$ on the contact condition.

When $\theta=1$, optimal convergence is obtained for both $H^{1}(\Omega)$ and weighted $L^{2}\left(\Gamma_{C}\right)$-norms of the error, but only for the largest value of the parameter $\gamma_{0}\left(\gamma_{0}=100 E\right)$. This corroborates the theoretical result of Theorem 3 for which the optimal rate of convergence is obtained for a sufficiently large $\gamma_{0}$. When $\theta=0$, for the smallest value of $\gamma_{0}$ the convergence remains sub-optimal. However, for the intermediate value of $\gamma_{0}\left(\gamma_{0}=E\right)$ the optimal convergence is 
reached. Concerning the version with $\theta=-1$, which corresponds to an unconditionally coercive problem, one can see that optimal convergence is reached for all values of $\gamma_{0}$.

Remark that the same conclusions hold both for 2D and 3D cases, and linear and quadratic finite elements, though the difference between the variants $\theta=1$ and the others can be greater for quadratic elements or in the 3D case. A strategy to guarantee an optimal convergence is of course to consider a sufficiently large $\gamma_{0}$. However, the price to pay is an ill-conditioned discrete problem. The study presented in [81] for the versions $\theta=1$ and $\theta=0$ shows that Newton's method has important difficulties to converge when $\gamma_{0}$ is large. When symmetry is not required, a better strategy seems to consider the version with $\theta=-1$ or an intermediate value of $\theta=0$ which ensure both a optimal convergence rate and few iterations of Newton's method to converge.

\subsection{A posteriori error estimation}

We report the test case taken from [57] (see also [56, 72] in the frictional case). We consider the domain $\Omega=$ $(0,1) \times(0,1)$ with material characteristics $E=10^{6}$ and $P=0.3$. A homogeneous Dirichlet condition on $\Gamma_{D}=$ $\{0\} \times(0,1)$ is prescribed to clamp the body. The body is potentially in contact on $\Gamma_{C}=\{1\} \times(0,1)$ with a rigid obstacle and $\Gamma_{N}=(0,1) \times(\{0\} \cup\{1\})$ is the location of a homogeneous Neumann condition. The body $\Omega$ is acted on by a vertical volume density of force $\mathbf{f}=\left(0, f_{2}\right)$ with $f_{2}=-76518$ such that there is coexistence of a slip zone and a separation zone with a transition point between both zones. For error computations, since we do not have a closed-form solution, a reference solution is computed with Lagrange $\mathbb{P}_{2}$ elements, $h=1 / 160, \gamma_{0}=E$ and $\theta=-1$. First of all we illustrate in Fig. 3 the difference between uniform and adaptive refinement. For the latter we refine only the mesh elements $T$ in which the local estimator $\eta_{T}$ is below a given threshold $s=2.5 \times 10^{-3}$. The minimal (respectively maximal) size of the adaptive mesh is equal to $1 / 160$ (respectively $h=1 / 40$ ). As expected the rate of convergence with respect to the number of degrees of freedom is far better in the case of adaptive refinement than with uniform refinement.

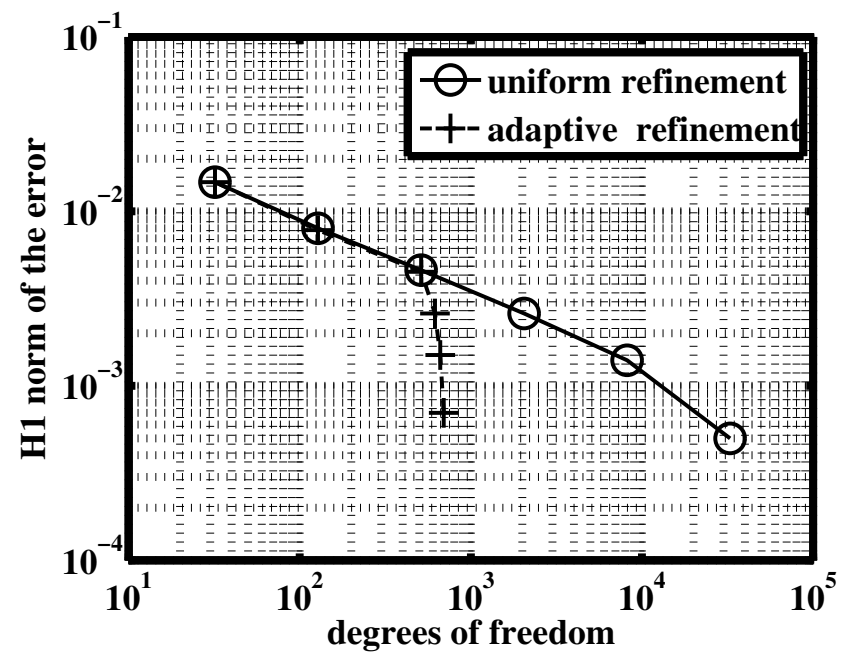

Fig. 3 Rate of convergence for uniform and adaptive refinement methods. Parameters $\gamma_{0}=E, \theta=-1$ and Lagrange $\mathbb{P}_{2}$ elements. 

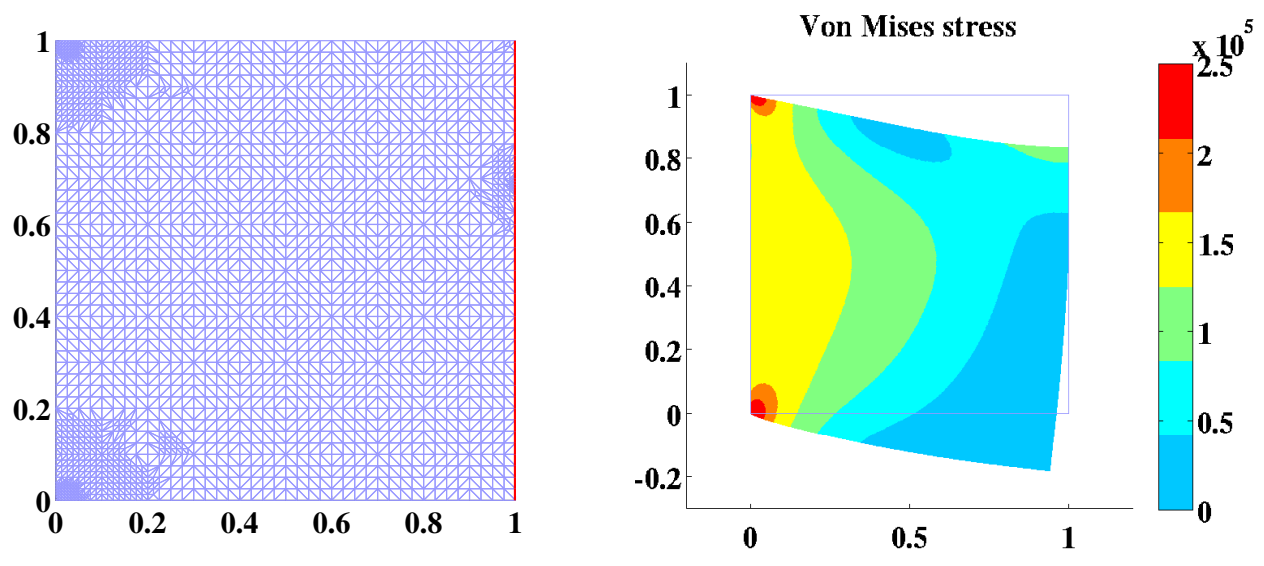

Fig. 4 Left panel: mesh with adaptive refinement and contact boundary on the right. Right panel: plot of Von Mises stress. Parameters $\gamma_{0}=E, \theta=-1$ and Lagrange $\mathbb{P}_{2}$ elements.

The solution obtained with adaptive refinement and $\theta=-1$ is depicted in Fig. 4. We observe that the error is concentrated at both left corners (transition between Dirichlet and Neumann conditions) and near the transition point between contact and separation.

A detailed numerical convergence study of the error estimator $\eta$ for this test-case, as well as for 2D and 3D Hertz contact, is provided in [22].

\section{Recent extensions}

This section is about recent extensions. We describe numerical approximations of the contact between two elastic bodies, first with the master/slave (biased) formulation in 5.1, where the meshes are conformal to the geometry, and in 5.2 with cut-finite elements and fictitious domains. An unbiased formulation is provided in 5.3. Finally the extension to elastodynamics is given in 5.4.

\subsection{Contact between two elastic bodies}

Now, we consider two elastic bodies $\Omega^{1}$ and $\Omega^{2}$ expected to come into contact. To simplify notations, a general index $i$ is used to represent indifferently each body $(i=1,2)$. We denote by $\Gamma_{C}^{i}$ a portion of the boundary of the body $\Omega^{i}$ which is a candidate contact surface with an outward unit normal vector $\mathbf{n}^{i}$.

For the contact surfaces, let us assume a sufficiently smooth one to one application (projection for instance) mapping each point of the first contact surface to a point of the second one (see also Fig. 5):

$$
\Pi^{1}: \Gamma_{C}^{1} \rightarrow \Gamma_{C}^{2} .
$$

Let $J^{1}$ be the Jacobian of the transformation $\Pi^{1}$ and $J^{2}=\frac{1}{J^{1}}$ the Jacobian of $\Pi^{2}=\left(\Pi^{1}\right)^{-1}$.

We suppose in the following that $J^{1}>0$. We define on each contact surface another "normal" vector $\tilde{\mathbf{n}}^{i}$ such that: 


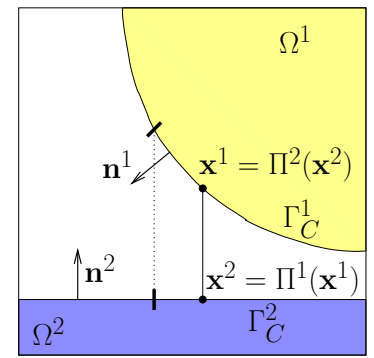

Fig. 5 Two bodies $\Omega^{1}$ and $\Omega^{2}$ in contact: mapping $\Pi^{1}$ from $\Gamma_{C}^{1}$ to $\Gamma_{C}^{2}$ and its inverse mapping $\Pi^{2}$.

$$
\tilde{\mathbf{n}}^{i}(\mathbf{x})= \begin{cases}\frac{\Pi^{i}(\mathbf{x})-\mathbf{x}}{\left\|\Pi^{i}(\mathbf{x})-\mathbf{x}\right\|} & \text { if } \mathbf{x} \neq \Pi^{i}(\mathbf{x}), \\ \mathbf{n}^{i} & \text { if } \mathbf{x}=\Pi^{i}(\mathbf{x}) .\end{cases}
$$

Note that $\tilde{\mathbf{n}}^{1}=-\tilde{\mathbf{n}}^{2} \circ \Pi^{1}$ and $\tilde{\mathbf{n}}^{2}=-\tilde{\mathbf{n}}^{1} \circ \Pi^{2}$. For any displacement field $\mathbf{v}^{i}$ and for any density of surface forces $\sigma^{i}\left(\mathbf{v}^{i}\right) \mathbf{n}^{i}$ defined on $\partial \Omega_{i}$, we adopt the following notation:

$$
\mathbf{v}^{i}=v_{n}^{i} \tilde{\mathbf{n}}^{i}+\mathbf{v}_{\mathbf{t}}^{i} \text { and } \sigma^{i}\left(\mathbf{v}^{i}\right) \mathbf{n}^{i}=\sigma_{n}^{i}\left(\mathbf{v}^{i}\right) \tilde{\mathbf{n}}^{i}+\sigma_{\mathbf{t}}^{i}\left(\mathbf{v}^{i}\right) .
$$

The two-body contact problem, in the linear elastic framework, consists in finding the displacement field $\mathbf{u}=$ $\left(\mathbf{u}^{1}, \mathbf{u}^{2}\right)$ verifying the equations (38) and the contact conditions described hereafter:

$$
\begin{aligned}
\operatorname{div} \sigma^{i}\left(\mathbf{u}^{i}\right)+\mathbf{f}^{i} & =\mathbf{0} & & \text { in } \Omega^{i}, \\
\sigma^{i}\left(\mathbf{u}^{i}\right) & =\mathbf{C}^{i} \varepsilon\left(\mathbf{u}^{i}\right) & & \text { in } \Omega^{i}, \\
\mathbf{u}^{i} & =\mathbf{0} & & \text { on } \Gamma_{D}^{i}, \\
\sigma^{i}\left(\mathbf{u}^{i}\right) \mathbf{n}^{i} & =\mathbf{f}_{N}^{i} & & \text { on } \Gamma_{N}^{i},
\end{aligned}
$$

We consider a zero initial normal gap to simplify the notations (see [39] for a nonzero one) and we define the relative normal displacements $\llbracket u \rrbracket_{n}^{1}=\left(\mathbf{u}^{1}-\mathbf{u}^{2} \circ \Pi^{1}\right) \cdot \tilde{\mathbf{n}}^{1}$ and $\llbracket u \rrbracket_{n}^{2}=\left(\mathbf{u}^{2}-\mathbf{u}^{1} \circ \Pi^{2}\right) \cdot \tilde{\mathbf{n}}^{2}$.

The classical master/slave (biased) formulation is obtained by selecting for instance $\Gamma_{C}^{1}$ to be the slave surface and $\Gamma_{C}^{2}$ to be the master one. Then the unilateral contact condition is written on the slave side $\Gamma_{C}^{1}$ :

$$
\llbracket u \rrbracket_{n}^{1} \leq 0, \quad \sigma_{n}^{1}\left(\mathbf{u}^{1}\right) \leq 0, \quad \sigma_{n}^{1}\left(\mathbf{u}^{1}\right) \llbracket u \rrbracket_{n}^{1}=0 .
$$

Let $s^{1} \in L^{2}\left(\Gamma_{C}^{1}\right), s^{1} \geq 0, \llbracket \mathbf{u} \rrbracket_{\mathbf{t}}^{1}=\mathbf{u}_{\mathbf{t}}^{1}-\mathbf{u}_{\mathbf{t}}^{2} \circ \Pi^{1}$. The Tresca friction condition on $\Gamma_{C}^{1}$ reads:

$$
\begin{cases}\left\|\sigma_{\mathbf{t}}^{1}\left(\mathbf{u}^{1}\right)\right\| \leq s^{1} & \text { if } \llbracket \mathbf{u} \rrbracket_{\mathbf{t}}^{1}=0, \\ \sigma_{\mathbf{t}}^{1}\left(\mathbf{u}^{1}\right)=-s^{1} \frac{\llbracket \mathbf{u} \rrbracket_{\mathbf{t}}^{1}}{\left\|\llbracket \mathbf{u} \rrbracket_{\mathbf{t}}^{1}\right\|} & \text { otherwise. }\end{cases}
$$

As in Section 2, we reformulate the contact and friction conditions (39)-(40) as follows: 


$$
\begin{aligned}
& \sigma_{n}^{1}\left(\mathbf{u}^{1}\right)=\left[\sigma_{n}^{1}\left(\mathbf{u}^{1}\right)-\gamma^{1} \llbracket u \rrbracket_{n}^{1}\right]_{\mathbb{R}^{-}}, \\
& \sigma_{\mathbf{t}}^{1}\left(\mathbf{u}^{1}\right)=\left[\sigma_{\mathbf{t}}^{1}\left(\mathbf{u}^{1}\right)-\gamma^{1} \llbracket \mathbf{u} \rrbracket_{\mathfrak{t}}^{1}\right]_{s^{1}},
\end{aligned}
$$

where $\gamma^{1}$ is the counterpart of $\gamma$ on the slave surface $\Gamma_{C}^{1}$. We note $\mathscr{T}^{h, i}$ a triangulation of the domain $\Omega^{i}$ and introduce the finite element spaces as in Section 2:

$$
\mathbf{V}^{h}:=\mathbf{V}^{h, 1} \times \mathbf{V}^{h, 2}, \quad \text { with } \quad \mathbf{V}^{h, i}:=\left\{\mathbf{v}^{h, i} \in \mathscr{C}^{0}\left(\overline{\Omega^{i}}\right): \mathbf{v}_{\mid T}^{h, i} \in\left(\mathbb{P}_{k}(T)\right)^{d}, \forall T \in \mathscr{T}^{h, i}, \mathbf{v}^{h, i}=\mathbf{0} \text { on } \Gamma_{D}^{i}\right\} .
$$

We use the Green formula and equations (38a)-(38d) as well as the Nitsche's writing of the contact and friction conditions (41)-(42) to get the following biased finite element approximation:

$$
\left\{\begin{array}{l}
\text { Find } \mathbf{u}^{h} \in \mathbf{V}^{h} \text { such that, } \\
A_{\theta \gamma}^{1}\left(\mathbf{u}^{h}, \mathbf{v}^{h}\right)+\int_{\Gamma_{C}^{1}} \frac{1}{\gamma^{1}}\left[\mathrm{P}_{1, \gamma^{1}}^{1, \mathbf{n}}\left(\mathbf{u}^{h}\right)\right]_{\mathbb{R}^{-}} \mathrm{P}_{\theta, \gamma^{1}}^{1, \mathbf{n}}\left(\mathbf{v}^{h}\right) \mathrm{d} \Gamma \\
+\int_{\Gamma_{C}^{1}} \frac{1}{\gamma^{1}}\left[\mathbf{P}_{1, \gamma^{1}}^{1, \mathbf{t}}\left(\mathbf{u}^{h}\right)\right]_{s^{1}} \cdot \mathbf{P}_{\theta, \gamma^{1}}^{1, \mathbf{t}}\left(\mathbf{v}^{h}\right) \mathrm{d} \Gamma=L\left(\mathbf{v}^{h}\right), \quad \forall \mathbf{v}^{h} \in \mathbf{V}^{h},
\end{array}\right.
$$

where:

$$
\mathrm{P}_{\theta, \gamma^{i}}^{i, \mathbf{n}}(\mathbf{v}):=\theta \sigma_{n}^{i}\left(\mathbf{v}^{i}\right)-\gamma^{i} \llbracket v \rrbracket_{n}^{i},, \quad \quad \mathbf{P}_{\theta, \gamma^{i}}^{i, \mathbf{t}}(\mathbf{v}):=\theta \sigma_{\mathbf{t}}^{i}\left(\mathbf{v}^{i}\right)-\gamma^{i} \llbracket \rrbracket_{\mathbf{t}}^{i},
$$

and

$$
\begin{aligned}
A_{\theta \gamma}^{1}(\mathbf{u}, \mathbf{v}) & :=\sum_{i=1}^{2}\left(\int_{\Omega^{i}} \sigma\left(\mathbf{u}^{i}\right): \varepsilon\left(\mathbf{v}^{i}\right) d \Omega\right)-\int_{\Gamma_{C}^{1}} \frac{\theta}{\gamma^{1}} \sigma^{1}\left(\mathbf{u}^{1}\right) \mathbf{n} \cdot \sigma^{1}\left(\mathbf{v}^{1}\right) \mathbf{n} \mathrm{d} \Gamma, \\
L(\mathbf{v}) & :=\sum_{i=1}^{2}\left(\int_{\Omega^{i}} \mathbf{f}^{i} \cdot \mathbf{v}^{i} d \Omega+\int_{\Gamma_{N}^{i}} \mathbf{f}_{N}^{i} \cdot \mathbf{v}^{i} d \Gamma\right) .
\end{aligned}
$$

All the mathematical properties presented in Section 3 for the deformable/rigid case can be transposed to this biased deformable/deformable Nitsche formulation (see [39]).

\subsection{Contact between two elastic bodies with a fictitious domain}

A complete analysis in a frictionless case is presented in [39] in the framework of a fictitious domain discretization using cut-elements. A fictitious domain $\Omega$ that contains both $\Omega^{1}$ and $\Omega^{2}$ is considered, generally with a simple geometry such that a structured mesh $\mathscr{T}^{h}$ of $\Omega$ can be used (see the example in Fig. 6). Then a single finite element space

$$
\mathbf{W}^{h}:=\left\{\mathbf{v}^{h} \in \mathscr{C}^{0}(\bar{\Omega}): \mathbf{v}_{\mid T}^{h, i} \in\left(\mathbb{P}_{k}(T)\right)^{d}, \forall T \in \mathscr{T}^{h}\right\}
$$

is used to approximate the displacement of the two bodies, in the sense that we consider the following approximations space

$$
\mathbf{V}^{h}:=\mathbf{W}_{\mid \Omega^{1}}^{h} \times \mathbf{W}_{\mid \Omega^{2}}^{h}
$$




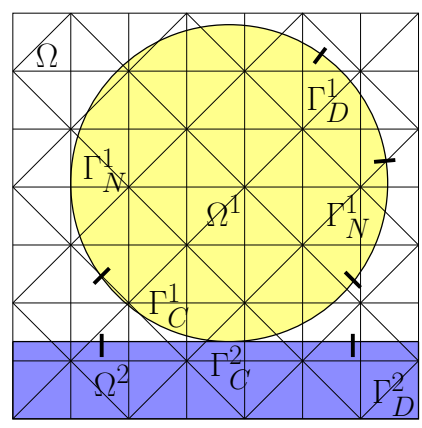

Fig. 6 Two bodies $\Omega^{1}$ and $\Omega^{2}$ in contact: a single mesh of the fictitious domain $\Omega$.

in the discrete problem (43), where $\left.\mathbf{W}^{h}\right|_{\Omega^{i}}$ is the space of restrictions to $\Omega^{i}$ of functions of $\mathbf{W}^{h}$. Additionnaly, since the boundaries of the bodies are independent of the mesh element edges, the Dirichlet conditions are also prescribed with Nitsche's method. This yields the following approximation (in the frictionless case):

$$
\left\{\begin{array}{l}
\text { Find } \mathbf{u}^{h} \in \mathbf{V}^{h} \text { such that, } \\
\bar{A}_{\theta \gamma}^{1}\left(\mathbf{u}^{h}, \mathbf{v}^{h}\right)+\int_{\Gamma_{C}^{1}} \frac{1}{\gamma^{1}}\left[\overline{\mathrm{P}}_{1, \gamma^{1}}^{1, \mathbf{n}}\left(\mathbf{u}^{h}\right)\right]_{\mathbb{R}^{-}} \overline{\mathrm{P}}_{\theta, \gamma^{1}}^{1, \mathbf{n}}\left(\mathbf{v}^{h}\right) \mathrm{d} \Gamma \\
+\sum_{i=1,2} \int_{\Gamma_{D}^{i}}\left(\frac{1}{\gamma^{1}} \mathbf{u}^{h, i} \cdot \mathbf{v}^{h, i}-R_{\hat{\rho}}\left(\mathbf{u}^{h, i}\right) \cdot \mathbf{v}^{h, i}-\theta \mathbf{u}^{h, i} \cdot R_{\hat{\rho}}\left(\mathbf{v}^{h, i}\right)\right) \mathrm{d} \Gamma=L\left(\mathbf{v}^{h}\right), \quad \forall \mathbf{v}^{h} \in \mathbf{V}^{h},
\end{array}\right.
$$

where now

$$
\overline{\mathrm{P}}_{\theta, \gamma^{i}}^{i, \mathbf{n}}(\mathbf{v}):=\theta R_{\hat{\rho}}\left(\mathbf{v}^{i}\right)-\gamma^{i} \llbracket v \rrbracket_{n}^{i}
$$

and

$$
\bar{A}_{\theta \gamma}^{1}(\mathbf{u}, \mathbf{v}):=\sum_{i=1}^{2}\left(\int_{\Omega^{i}} \sigma\left(\mathbf{u}^{i}\right): \varepsilon\left(\mathbf{v}^{i}\right) d \Omega\right)-\int_{\left(\Gamma_{C}^{1} \cup \Gamma_{D}^{1} \cup \Gamma_{D}^{2}\right)} \frac{\theta}{\gamma^{1}} R_{\hat{\rho}}\left(\mathbf{u}^{1}\right) \cdot R_{\hat{\rho}}\left(\mathbf{v}^{1}\right) \mathrm{d} \Gamma
$$

The new term $R_{\hat{\rho}}\left(\mathbf{v}^{h, i}\right)$ is an approximation of $\sigma^{i}\left(\mathbf{v}^{i}\right) \mathbf{n}^{i}$ built in order to recover an optimal order of convergence. In [39] this operator is defined as the polynomial extension of the displacement field of neighbour elements having a sufficiently large intersection with the domain $\Omega^{i}$, as proposed initially in [52]. Note that an alternative is the use of the so-called ghost penalty introduced in [15].

Remark finally that the presentation here differs slightly from [39] in which the second Newton's law is used to reformulate differently the contact conditions (39). This second Newton's law reveals as well to be a key ingredient for the derivation of an unbiased formulation, as detailed in next section.

\subsection{Unbiased formulation for self-and multi-body contact}

If the master/slave formulation consists in a natural extension of the contact treatment between a deformable body and a rigid ground, it has no complete theoretical justification, and induces detection difficulties in the case of self-contact and multi-body contact. We provide in this section a short description of the unbiased formulation 
presented in [29] that circumvents these difficulties. We do not distinguish between a master surface and a slave one since we impose the non-penetration and the friction conditions on both of them. Unbiased contact and friction formulations have been considered before in [83] and references therein.

In order to obtain an unbiased method we prescribe the contact condition on the two surfaces in a symmetric way. Thus, the conditions describing contact on $\Gamma_{C}^{i}(i=1,2)$ are:

$$
\llbracket u \rrbracket_{n}^{i} \leq 0, \quad \sigma_{n}^{i}\left(\mathbf{u}^{i}\right) \leq 0, \quad \sigma_{n}^{i}\left(\mathbf{u}^{i}\right) \llbracket u \rrbracket_{n}^{i}=0 .
$$

Let $s^{i} \in L^{2}\left(\Gamma_{C}^{i}\right), s^{i} \geq 0, \llbracket \mathbf{u} \rrbracket_{\mathfrak{t}}^{1}=\mathbf{u}_{\mathbf{t}}^{1}-\mathbf{u}_{\mathfrak{t}}^{2} \circ \Pi^{1}$ and $\llbracket \mathbf{u} \rrbracket_{\mathfrak{t}}^{2}=\mathbf{u}_{\mathfrak{t}}^{2}-\mathbf{u}_{\mathbf{t}}^{1} \circ \Pi^{2}=-\llbracket \mathbf{u} \rrbracket_{\mathfrak{t}}^{1} \circ \Pi^{2}$. The Tresca friction condition on $\Gamma_{C}^{1}$ and $\Gamma_{C}^{2}$ reads:

$$
\begin{cases}\left\|\sigma_{\mathfrak{t}}^{i}\left(\mathbf{u}^{i}\right)\right\| \leq s^{i} & \text { if } \llbracket \mathbf{u} \rrbracket_{\mathfrak{t}}^{i}=0, \\ \sigma_{\mathfrak{t}}^{i}\left(\mathbf{u}^{i}\right)=-s^{i} \frac{\llbracket \mathbf{u} \rrbracket_{\mathfrak{t}}^{i}}{\left\|\llbracket \mathbf{u} \rrbracket_{\mathfrak{t}}^{i}\right\|} & \text { otherwise. }\end{cases}
$$

Finally, we need to consider the second Newton law between the two bodies:

$$
\left\{\begin{array}{l}
\int_{\gamma_{C}^{1}} \sigma_{n}^{1}\left(\mathbf{u}^{1}\right) \mathrm{d} s-\int_{\gamma_{C}^{2}} \sigma_{n}^{2}\left(\mathbf{u}^{2}\right) \mathrm{d} s=0, \\
\int_{\gamma_{C}^{1}} \sigma_{\mathbf{t}}^{1}\left(\mathbf{u}^{1}\right) \mathrm{d} s+\int_{\gamma_{C}^{2}} \sigma_{\mathbf{t}}^{2}\left(\mathbf{u}^{2}\right) \mathrm{d} s=\mathbf{0},
\end{array}\right.
$$

where $\gamma_{C}^{1}$ is any subset of $\Gamma_{C}^{1}$ and $\gamma_{C}^{2}=\Pi^{1}\left(\gamma_{C}^{1}\right)$. Mapping all terms on $\gamma_{C}^{1}$ allows writing:

$$
\left\{\begin{array}{l}
\sigma_{n}^{1}\left(\mathbf{u}^{1}\right)-J^{1} \sigma_{n}^{2}\left(\mathbf{u}^{2} \circ \Pi^{1}\right)=0, \\
\sigma_{\mathbf{t}}^{1}\left(\mathbf{u}^{1}\right)+J^{1} \sigma_{\mathbf{t}}^{2}\left(\mathbf{u}^{2} \circ \Pi^{1}\right)=\mathbf{0},
\end{array} \text { on } \Gamma_{C}^{1} .\right.
$$

Remark 13. : A similar condition holds on $\Gamma_{C}^{2}$ :

$$
\left\{\begin{array}{l}
\sigma_{n}^{2}\left(\mathbf{u}^{2}\right)-J^{2} \sigma_{n}^{1}\left(\mathbf{u}^{1} \circ \Pi^{2}\right)=0, \\
\sigma_{\mathbf{t}}^{2}\left(\mathbf{u}^{2}\right)+J^{2} \sigma_{\mathbf{t}}^{1}\left(\mathbf{u}^{1} \circ \Pi^{2}\right)=\mathbf{0} .
\end{array}\right.
$$

Let us mention that, due to second Newton law, we need to fix $s^{1}$ and $s^{2}$ such that:

$$
-s^{1} \frac{\llbracket \mathbf{u} \rrbracket_{\mathfrak{t}}^{1}}{\left\|\llbracket \mathbf{u} \rrbracket_{\mathfrak{t}}^{1}\right\|}=\sigma_{\mathfrak{t}}^{1}\left(\mathbf{u}^{1}\right)=-J^{1} \sigma_{\mathfrak{t}}^{2}\left(\mathbf{u}^{2} \circ \Pi^{1}\right)=J^{1} s^{2} \frac{\llbracket \mathbf{u} \rrbracket_{\mathfrak{t}}^{2} \circ \Pi^{1}}{\left\|\llbracket \mathbf{u} \rrbracket_{\mathfrak{t}}^{2} \circ \Pi^{1}\right\|}=-J^{1} s^{2} \frac{\llbracket \mathbf{u} \rrbracket_{\mathfrak{t}}^{1}}{\left|\llbracket \mathbf{u} \rrbracket_{\mathfrak{t}}^{1}\right|} .
$$

It results that the following compatibility condition on $s^{1}$ and $s^{2}$ needs to be satisfied:

$$
s^{1}=J^{1} s^{2} .
$$

The reformulation of the contact and friction conditions (45)-(46) reads:

$$
\begin{aligned}
& \sigma_{n}^{i}\left(\mathbf{u}^{i}\right)=\left[\sigma_{n}^{i}\left(\mathbf{u}^{i}\right)-\gamma^{i} \llbracket u \rrbracket_{n}^{i}\right]_{\mathbb{R}^{-}}, \\
& \sigma_{\mathbf{t}}^{i}\left(\mathbf{u}^{i}\right)=\left[\sigma_{\mathbf{t}}^{i}\left(\mathbf{u}^{i}\right)-\gamma^{i} \llbracket \mathbf{u} \rrbracket_{\mathbf{t}}^{i}\right]_{s^{i}},
\end{aligned}
$$


where $\gamma^{i}$ plays the same role as $\gamma$ on each contact surface $\Gamma_{C}^{i}$. Still using the Green formula and the different equations considered as well as the Nitsche's writing of the contact and friction we obtain the following unbiased finite element approximation:

$$
\left\{\begin{array}{l}
\text { Find } \mathbf{u}^{h} \in \mathbf{V}^{h} \text { such that, } \\
A_{\theta \gamma}^{1,2}\left(\mathbf{u}^{h}, \mathbf{v}^{h}\right)+\frac{1}{2} \sum_{i=1,2} \int_{\Gamma_{C}^{i}} \frac{1}{\gamma^{i}}\left[\mathrm{P}_{1, \gamma^{i}}^{i, \mathbf{n}}\left(\mathbf{u}^{h}\right)\right]_{\mathbb{R}^{-}} \mathrm{P}_{\theta, \gamma^{i}}^{i, \mathbf{n}}\left(\mathbf{v}^{h}\right) \mathrm{d} \Gamma \\
+\frac{1}{2} \sum_{i=1,2} \int_{\Gamma_{C}^{i}} \frac{1}{\gamma^{i}}\left[\mathbf{P}_{1, \gamma^{i}}^{i, \mathbf{t}}\left(\mathbf{u}^{h}\right)\right]_{s^{i}} \cdot \mathbf{P}_{\theta, \gamma^{i}}^{i, \mathbf{t}}\left(\mathbf{v}^{h}\right) \mathrm{d} \Gamma=L\left(\mathbf{v}^{h}\right), \quad \forall \mathbf{v}^{h} \in \mathbf{V}^{h},
\end{array}\right.
$$

where this time:

$$
A_{\theta \gamma}^{1,2}(\mathbf{u}, \mathbf{v}):=\sum_{i=1}^{2}\left(\int_{\Omega^{i}} \sigma\left(\mathbf{u}^{i}\right): \varepsilon\left(\mathbf{v}^{i}\right) d \Omega-\frac{1}{2} \int_{\Gamma_{C}^{i}} \frac{\theta}{\gamma^{i}} \sigma^{i}\left(\mathbf{u}^{i}\right) \mathbf{n} \cdot \sigma^{i}\left(\mathbf{v}^{i}\right) \mathbf{n} \mathrm{d} \Gamma\right) .
$$

Note that in the above method the two contact surfaces are treated similarly. This small strain formulation allows an extended mathematical study which is impossible to perform in a large transformations framework. Indeed, all the mathematical analysis carried out for the unilateral contact problem can be adapted for this method, and yields the same theoretical properties of well-posedness and convergence. This analysis as well as a complete numerical study in the small strain framework can be found in [29].

However, the true potential of this unbiased paradigm is to able the design of a similar method in large transformations. We quickly describe it in the rest of this section (see [74] for more details). Let $\Omega \subset \mathbb{R}^{d}$ be a potential multi-body domain representing the reference configuration of one or several hyper-elastic bodies. Let $\Gamma_{C}$ be the part of the boundary of $\Omega$ where contact may occur. Let $\mathscr{J}_{\mathrm{H}}(\cdot)$ be the potential of the hyper-elastic law (more details can be found in [80]). Then, to each (material) point $\mathbf{x} \in \Gamma_{C}$ and to the displacement $\mathbf{u}: \Omega \rightarrow \mathbb{R}^{d}$, we associate $g(\mathbf{x}, \mathbf{u})$ the (nonnegative) gap distance (in the deformed configuration) between the surface $\Gamma_{C}$ at point $\mathbf{x}$ and the nearest other surface in potential contact. The main difference with the small strain case is that the point in potential contact at $\mathbf{x} \in \Gamma_{C}$ is a priori unknown. Moreover it changes during the deformation and may be quite difficult to define. This gap calculation can be performed in different ways, see [80] and the references therein for more details.

Then, an extension of unbiased Nitsche's method to large transformation contact in the frictionless case can be written

$$
\left\langle D \mathscr{J}_{\mathrm{H}}\left(\mathbf{u}^{h}\right), \mathbf{v}^{h}\right\rangle+\frac{1}{2} \int_{\Gamma_{C}}\left[\sigma_{n}\left(\mathbf{u}^{h}\right)+\gamma g\left(\mathbf{u}^{h}\right)\right]_{\mathbb{R}^{-}}\left\langle D g\left(\mathbf{u}^{h}\right), \mathbf{v}^{h}\right\rangle d \Gamma=0,
$$

for all admissible increment of displacement $\mathbf{v}^{h}$. The notation $\sigma_{n}(\mathbf{u})$ stands for the contact stress in large transformations.

Note that, similarly to the method presented in the small strain case, the integral on $\Gamma_{C}$ in (52) being calculated on the whole boundary $\Gamma_{C}$, it is notably calculated on both the two corresponding surfaces of a potential contact whether a multi-body contact is considered or a self-contact. Nitsche's method allows thus to approximate both multi-body- and self-contact in the same simple formalism. An example of application of this method to the contact and self-contact of two deformable tubes is presented in Fig. 7. 

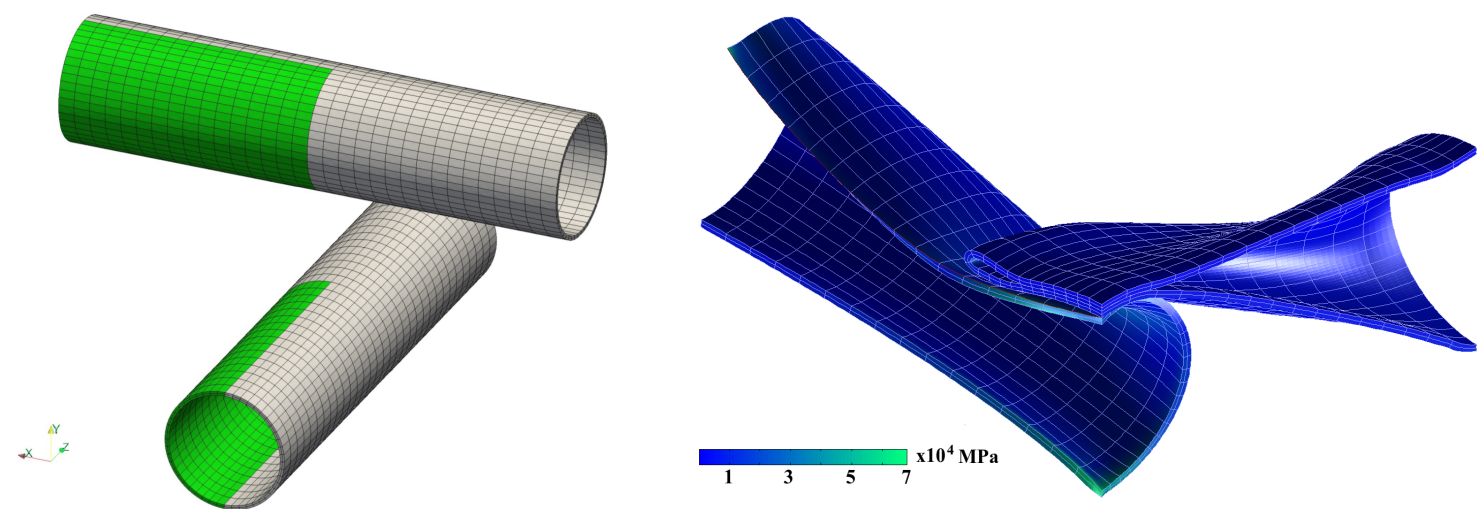

Fig. 7 Example of use of the unbiased Nitsche's method for the contact with large transformations of two tubes. Only a quarter of each tube is taken into account. Left panel : geometry and mesh in undeformed configuration. Right panel : deformed configuration with Von Mises contour plot.

\subsection{Contact in elastodynamics}

This section shows an adaptation of Nitsche's method to elastodynamic frictionless contact problems. In this context Nitsche-based approximation produces well-posed space semi-discretizations contrary to standard finite element discretizations. We recall the main results of the analysis for the space semi-discretization in terms of well-posedness and energy conservation. We consider some time-marching schemes (theta-scheme, Newmark and a new hybrid scheme) for which the well-posedness and the stability results are given. The details can be found in the papers [26] and [27]. This sections ends with an extension of the method to the frictional case which is actually under investigation in [25].

The setting is the same as in section 2, but in the evolution case: an elastic body $\Omega$ in $\mathbb{R}^{d}$ with $d=2,3$ of density $\rho$ (constant to simplify). In the reference configuration, the body is in contact on $\Gamma_{C}$ with a rigid foundation and we suppose that the unknown contact zone during deformation is included into $\Gamma_{C}$.

We consider the unilateral contact problem in linear elastodynamics during a time interval $[0, T)$ where $T>0$ is the final time. We denote by $\Omega_{T}:=(0, T) \times \Omega$ the time-space domain, and similarly $\Gamma_{D T}:=(0, T) \times \Gamma_{D}, \Gamma_{N T}:=$ $(0, T) \times \Gamma_{N}$ and $\Gamma_{C T}:=(0, T) \times \Gamma_{C}$. The problem consists in finding the displacement field $\mathbf{u}:[0, T) \times \Omega \rightarrow \mathbb{R}^{d}$ verifying the equations and conditions (53)-(54):

$$
\begin{aligned}
& \operatorname{div} \sigma(\mathbf{u})+\mathbf{f}=\rho \ddot{\mathbf{u}}, \quad \sigma(\mathbf{u})=\mathbf{C} \varepsilon(\mathbf{u}) \quad \text { in } \Omega_{T}, \\
& \mathbf{u}=\mathbf{0} \quad \text { on } \Gamma_{D T}, \\
& \sigma(\mathbf{u}) \mathbf{n}=\mathbf{f}_{N} \quad \text { on } \Gamma_{N T}, \\
& \mathbf{u}(0, \cdot)=\mathbf{u}_{0}, \quad \dot{\mathbf{u}}(0, \cdot)=\dot{\mathbf{u}}_{0} \quad \text { in } \Omega,
\end{aligned}
$$

where $\dot{\mathbf{u}}$ is the velocity of the elastic body and $\ddot{\mathbf{u}}$ its acceleration; $\mathbf{u}_{0}$ is the initial displacement and $\dot{\mathbf{u}}_{0}$ is the initial velocity.

The conditions describing unilateral contact without friction on $\Gamma_{C T}$ are:

$$
u_{n} \leq 0, \quad \sigma_{n}(\mathbf{u}) \leq 0, \quad \sigma_{n}(\mathbf{u}) u_{n}=0, \quad \sigma_{\mathbf{t}}(\mathbf{u})=\mathbf{0} .
$$


Note additionally that the initial displacement $\mathbf{u}_{0}$ should satisfy the compatibility condition $u_{0 n} \leq 0$ on $\Gamma_{C}$.

To our knowledge, the well-posedness of Problem (53)-(54) is still an open issue. For partial results, see e.g., [70, 66, 31, 37].

Remark 14. The (total) mechanical energy associated with the solution $\mathbf{u}$ to the dynamic contact problem (53)-(54) is:

$$
E(t):=\frac{1}{2} \rho\|\dot{\mathbf{u}}(t)\|_{0, \Omega}^{2}+\frac{1}{2} a(\mathbf{u}(t), \mathbf{u}(t)), \quad \forall t \in[0, T] .
$$

Formally, we get from (53), after multiplication by $\dot{\mathbf{u}}(t)$, integration by parts, with the boundary conditions on $\Gamma_{D T}$, $\Gamma_{N T}$, the absence of friction and the persistency condition $\sigma_{n}(\mathbf{u}(t)) \dot{\mathbf{u}}(t)=0$ (see, e.g., [69, 4, 53]) :

$$
\frac{d}{d t} E(t)=L(t) \dot{\mathbf{u}}(t), \quad \forall t \in[0, T] .
$$

In particular, when $L$ vanishes, we get energy conservation: $E(t)=E(0)$, for all $t \in[0, T]$.

\subsubsection{Semi-discretization in space with a Nitsche-based finite element method}

We consider the same family of finite element spaces $\mathbf{V}^{h}$ as defined in Section 2.2. The space semi-discretized Nitsche-based method for unilateral contact problems in elastodynamics then reads:

$$
\left\{\begin{array}{l}
\text { Find } \mathbf{u}^{h}:[0, T] \rightarrow \mathbf{V}^{h} \text { such that for } t \in[0, T]: \\
\left(\rho \ddot{\mathbf{u}}^{h}(t), \mathbf{v}^{h}\right)_{0, \Omega}+A_{\theta \gamma}^{\mathbf{n}}\left(\mathbf{u}^{h}(t), \mathbf{v}^{h}\right)+\int_{\Gamma_{C}} \frac{1}{\gamma}\left[\mathrm{P}_{1, \gamma}^{\mathbf{n}}\left(\mathbf{u}^{h}(t)\right)\right]_{\mathbb{R}^{-}} \mathrm{P}_{\theta, \gamma}^{\mathbf{n}}\left(\mathbf{v}^{h}\right) d \Gamma=L(t)\left(\mathbf{v}^{h}\right), \quad \forall \mathbf{v}^{h} \in \mathbf{V}^{h}, \\
\mathbf{u}^{h}(0, \cdot)=\mathbf{u}_{0}^{h}, \quad \dot{\mathbf{u}}^{h}(0, \cdot)=\dot{\mathbf{u}}_{0}^{h},
\end{array}\right.
$$

where $\mathbf{u}_{0}^{h}$ (resp. $\dot{\mathbf{u}}_{0}^{h}$ ) is a finite element approximation in $\mathbf{V}^{h}$ of the initial displacement $\mathbf{u}_{0}$ (resp. the initial velocity $\left.\dot{\mathbf{u}}_{0}\right)$.

Nitsche's formulation leads to a well-posed (Lipschitz) system of differential equations, as it will be shown below. This feature is shared with the standard penalty method, the difference being that Nitsche's method remains consistent (for details concerning consistency, we refer the reader to [26]).

Since we consider the frictionless case, we modify slightly the definition of $(\cdot, \cdot)_{\gamma}$ provided initially in Definition 2:

$$
\left(\mathbf{v}^{h}, \mathbf{w}^{h}\right)_{\gamma}:=\left(\mathbf{v}^{h}, \mathbf{w}^{h}\right)_{1, \Omega}+\left(\gamma^{\frac{1}{2}} v_{n}^{h}, \gamma^{\frac{1}{2}} w_{n}^{h}\right)_{0, \Gamma_{C}} .
$$

In order to prove well-posedness we reformulate (56) as a system of (non-linear) second-order differential equations. To this purpose, using Riesz's representation theorem in $\left(\mathbf{V}^{h},(\cdot, \cdot)_{\gamma}\right)$ we first introduce the mass operator $\mathbf{M}^{h}: \mathbf{V}^{h} \rightarrow \mathbf{V}^{h}$, which is defined for all $\mathbf{v}^{h}, \mathbf{w}^{h} \in \mathbf{V}^{h}$ by $\left(\mathbf{M}^{h} \mathbf{v}^{h}, \mathbf{w}^{h}\right)_{\gamma}=\left(\rho \mathbf{v}^{h}, \mathbf{w}^{h}\right)_{0, \Omega}$. Still using Riesz's representation theorem, we define the (non-linear) operator $\mathbf{B}^{h}: \mathbf{V}^{h} \rightarrow \mathbf{V}^{h}$, by means of the formula

$$
\left(\mathbf{B}^{h} \mathbf{v}^{h}, \mathbf{w}^{h}\right)_{\gamma}=A_{\theta \gamma}^{\mathbf{n}}\left(\mathbf{v}^{h}, \mathbf{w}^{h}\right)+\int_{\Gamma_{C}} \frac{1}{\gamma}\left[\mathrm{P}_{1, \gamma}^{\mathbf{n}}\left(\mathbf{v}^{h}\right)\right]_{\mathbb{R}^{-}} \mathrm{P}_{\theta, \gamma}^{\mathbf{n}}\left(\mathbf{w}^{h}\right) d \Gamma,
$$

for all $\mathbf{v}^{h}, \mathbf{w}^{h} \in \mathbf{V}^{h}$. Finally, we denote by $\mathbf{L}^{h}(t)$ the vector in $\mathbf{V}^{h}$ such that, for all $t \in[0, T]$ and for every $\mathbf{w}^{h}$ in $\mathbf{V}^{h}$ : $\left(\mathbf{L}^{h}(t), \mathbf{w}^{h}\right)_{\gamma}=L(t)\left(\mathbf{w}^{h}\right)$. With the above notation, Problem (56) reads: 


$$
\left\{\begin{array}{l}
\text { Find } \mathbf{u}^{h}:[0, T] \rightarrow \mathbf{V}^{h} \text { such that for } t \in[0, T]: \\
\mathbf{M}^{h} \ddot{\mathbf{u}}^{h}(t)+\mathbf{B}^{h} \mathbf{u}^{h}(t)=\mathbf{L}^{h}(t) \\
\mathbf{u}^{h}(0, \cdot)=\mathbf{u}_{0}^{h}, \quad \dot{\mathbf{u}}^{h}(0, \cdot)=\dot{\mathbf{u}}_{0}^{h}
\end{array}\right.
$$

The following theorem together with the boundedness of $\left\|\left(\mathbf{M}^{h}\right)^{-1}\right\|_{\gamma}$ (see [26]) show that Problem (56) (or equivalently Problem (57)) is well-posed.

Theorem 7. The operator $\mathbf{B}^{h}$ is Lipschitz-continuous in the following sense: there exists a constant $C>0$, independent of $h, \theta$ and $\gamma_{0}$ such that, for all $\mathbf{v}_{1}^{h}, \mathbf{v}_{2}^{h} \in \mathbf{V}^{h}$ :

$$
\left\|\mathbf{B}^{h} \mathbf{v}_{1}^{h}-\mathbf{B}^{h} \mathbf{v}_{2}^{h}\right\|_{\gamma} \leq C\left(1+\gamma_{0}^{-1}\right)(1+|\theta|)\left\|\mathbf{v}_{1}^{h}-\mathbf{v}_{2}^{h}\right\|_{\gamma} .
$$

As a consequence, for every value of $\theta \in \mathbb{R}$ and $\gamma_{0}>0$, Problem (56) admits one unique solution $\mathbf{u}^{h} \in \mathscr{C}^{2}\left([0, T], \mathbf{V}^{h}\right)$.

Remark 15. Note that, conversely to the static case (see $[23,28,21])$ and the fully-discrete case there is no condition on $\gamma_{0}$ for the space (semi-)discretization, which remains well-posed even if $\gamma_{0}$ is arbitrarily small.

Now we consider the energy estimates which are counterparts of the equation (55), in the semi-discretized case. Let us define the discrete energy as follows:

$$
E^{h}(t):=\frac{1}{2} \rho\left\|\dot{\mathbf{u}}^{h}(t)\right\|_{0, \Omega}^{2}+\frac{1}{2} a\left(\mathbf{u}^{h}(t), \mathbf{u}^{h}(t)\right), \quad \forall t \in[0, T] .
$$

which is associated to the solution $\mathbf{u}^{h}(t)$ to Problem (56). Note that this is the direct transposition of the mechanical energy $E(t)$ for the continuous system. Set also

$$
E_{\theta}^{h}(t):=E^{h}(t)-\frac{\theta}{2 \gamma_{0}}\left[\left\|\sigma_{n}\left(\mathbf{u}^{h}(t)\right)\right\|_{-\frac{1}{2}, h, \Gamma_{C}}^{2}-\left\|\left[\mathrm{P}_{1, \gamma}^{\mathbf{n}}\left(\mathbf{u}^{h}(t)\right)\right]_{\mathbb{R}^{-}}\right\|_{-\frac{1}{2}, h, \Gamma_{C}}^{2}\right]:=E^{h}(t)-\theta R^{h}(t),
$$

that corresponds to a modified energy in which a consistent term is added. This term denoted $R^{h}(t)$ represents, roughly speaking, the nonfulfillment of the contact condition (12) by $\mathbf{u}^{h}$.

Theorem 8. Suppose that the system associated to (53)-(54) is conservative, i.e., that $L(t) \equiv 0$ for all $t \in[0, T]$. The solution $\mathbf{u}^{h}$ to (56) then satisfies the following identity:

$$
\frac{d}{d t} E_{\theta}^{h}(t)=(1-\theta) \int_{\Gamma_{C}} \frac{1}{\gamma}\left[\mathrm{P}_{1, \gamma}^{\mathbf{n}}\left(\mathbf{u}^{h}(t)\right)\right]_{\mathbb{R}^{-}} \dot{u}_{n}^{h}(t) d \Gamma .
$$

Notably, when $\theta=1$, we get for any $t \in[0, T]: E_{1}^{h}(t)=E_{1}^{h}(0)$.

\subsubsection{Fully discrete formulations}

Now we fully discretize the dynamic contact problem by combining Nitsche's method with some classical schemes (theta-scheme, Newmark) as well as a new hybrid scheme. We focus on the well-posedness and the stability of the schemes.

Let $\tau>0$ be the time-step, and consider a uniform discretization of the time interval $[0, T]:\left(t^{0}, \ldots, t^{N}\right)$, with $t^{n}=n \tau, n=0, \ldots, N$. Let $\tilde{\theta} \in[0,1]$, we use the notation: 


$$
\mathbf{x}^{h, n+\tilde{\theta}}=(1-\tilde{\theta}) \mathbf{x}^{h, n}+\tilde{\theta} \mathbf{x}^{h, n+1}
$$

for arbitrary quantities $\mathbf{x}^{h, n}, \mathbf{x}^{h, n+1} \in \mathbf{V}^{h}$. Hereafter we denote by $\mathbf{u}^{h, n}$ (resp. $\dot{\mathbf{u}}^{h, n}$ and $\ddot{\mathbf{u}}^{h, n}$ ) the resulting discretized displacement (resp. velocity and acceleration) at time-step $t^{n}$. We next define the following energy:

$$
E^{h, n}:=\frac{1}{2} \rho\left\|\dot{\mathbf{u}}^{h, n}\right\|_{0, \Omega}^{2}+\frac{1}{2} a\left(\mathbf{u}^{h, n}, \mathbf{u}^{h, n}\right),
$$

which is associated with the solution $\mathbf{u}^{h, n}$ to Problems (59), (61) or (62). Set also

$$
E_{\theta}^{h, n}:=E^{h, n}-\frac{\theta}{2 \gamma_{0}}\left[\left\|\sigma_{n}\left(\mathbf{u}^{h, n}\right)\right\|_{-\frac{1}{2}, h, \Gamma_{C}}^{2}-\left\|\left[\mathrm{P}_{1, \gamma}^{\mathbf{n}}\left(\mathbf{u}^{h, n}\right)\right]_{\mathbb{R}^{-}}\right\|_{-\frac{1}{2}, h, \Gamma_{C}}^{2}\right]:=E^{h, n}-\theta R^{h, n} .
$$

Note that the energies $E^{h, n}$ and $E_{\theta}^{h, n}$ are the fully discrete counterparts of the semi-discrete energies $E^{h}(t)$ and $E_{\theta}^{h}(t)$.

- Theta-scheme. We discretize in time Problem (56) using a theta-scheme, of parameter $\tilde{\theta} \in[0,1]$. For $n \geq 0$, the fully discretized problem reads:

$$
\left\{\begin{array}{l}
\text { Find } \mathbf{u}^{h, n+1}, \dot{\mathbf{u}}^{h, n+1}, \ddot{\mathbf{u}}^{h, n+1} \in \mathbf{V}^{h} \text { such that: } \\
\mathbf{u}^{h, n+1}=\mathbf{u}^{h, n}+\tau \dot{\mathbf{u}}^{h, n+\tilde{\theta}} \\
\dot{\mathbf{u}}^{h, n+1}=\dot{\mathbf{u}}^{h, n}+\tau \ddot{\mathbf{u}}^{h, n+\tilde{\theta}} \\
\left(\rho \ddot{\mathbf{u}}^{h, n+1}, \mathbf{v}^{h}\right)_{0, \Omega}+A_{\theta \gamma}^{\mathbf{n}}\left(\mathbf{u}^{h, n+1}, \mathbf{v}^{h}\right)+\int_{\Gamma_{C}} \frac{1}{\gamma}\left[\mathrm{P}_{1, \gamma}^{\mathbf{n}}\left(\mathbf{u}^{h, n+1}\right)\right]_{\mathbb{R}^{-}} \mathrm{P}_{\theta, \gamma}^{\mathbf{n}}\left(\mathbf{v}^{h}\right) d \Gamma=L^{n+1}\left(\mathbf{v}^{h}\right), \quad \forall \mathbf{v}^{h} \in \mathbf{V}^{h},
\end{array}\right.
$$

with initial conditions $\mathbf{u}^{h, 0}=\mathbf{u}_{0}^{h}, \dot{\mathbf{u}}^{h, 0}=\dot{\mathbf{u}}_{0}^{h}, \ddot{\mathbf{u}}^{h, 0}=\ddot{\mathbf{u}}_{0}^{h}$ and where $L^{n+1}(\cdot)=L\left(t^{n+1}\right)(\cdot)$.

The following proposition concerns well-posedness and stability of the theta-scheme:

Proposition 3. I. If $\tilde{\theta}=0$, existence and uniqueness of (59) always holds since the scheme is fully explicit. 2. Let $\tilde{\boldsymbol{\theta}}>0$. If

$$
(1+\theta)^{2} \leq C \gamma_{0}\left(1+\frac{\rho h^{2}}{\tau^{2} \tilde{\theta}^{2}}\right)
$$

where $C$ is a positive constant, then at each time-step n, Problem (59) admits one unique solution.

3. Suppose that $L^{n} \equiv 0$ for all $n \geq 0$. Then, for $\gamma_{0}$ sufficiently large, $\theta=1$ and $\tilde{\theta}=1$ (backward Euler scheme), the following stability estimate holds for the solution to Problem (59), for all $n \geq 0$ :

$$
E_{1}^{h, n+1} \leq E_{1}^{h, n}
$$

Consequently, the scheme (59) is unconditionally stable when $\theta=1, \tilde{\theta}=1$ (i.e., stable for all $h>0$ and all $\tau>0$ ).

- Newmark scheme. We discretize in time Problem (56) using a Newmark scheme of parameters $\tilde{\beta} \in[0,1 / 2]$ and $\tilde{\gamma} \in[0,1]$. For $n \geq 0$, the fully discretized problem reads: 


$$
\left\{\begin{array}{l}
\text { Find } \mathbf{u}^{h, n+1}, \dot{\mathbf{u}}^{h, n+1}, \ddot{\mathbf{u}}^{h, n+1} \in \mathbf{V}^{h} \text { such that: } \\
\mathbf{u}^{h, n+1}=\mathbf{u}^{h, n}+\tau \dot{\mathbf{u}}^{h, n}+\frac{\tau^{2}}{2} \ddot{\mathbf{u}}^{h, n+2 \tilde{\beta}}, \\
\dot{\mathbf{u}}^{h, n+1}=\dot{\mathbf{u}}^{h, n}+\tau \ddot{\mathbf{u}}^{h, n+\tilde{\gamma}}, \\
\left(\rho \ddot{\mathbf{u}}^{h, n+1}, \mathbf{v}^{h}\right)_{0, \Omega}+A_{\theta \gamma}^{\mathbf{n}}\left(\mathbf{u}^{h, n+1}, \mathbf{v}^{h}\right)+\int_{\Gamma_{C}} \frac{1}{\gamma}\left[\mathrm{P}_{1, \gamma}^{\mathbf{n}}\left(\mathbf{u}^{h, n+1}\right)\right]_{\mathbb{R}^{-}} \mathrm{P}_{\theta, \gamma}^{\mathbf{n}}\left(\mathbf{v}^{h}\right) d \Gamma=L^{n+1}\left(\mathbf{v}^{h}\right), \quad \forall \mathbf{v}^{h} \in \mathbf{V}^{h},
\end{array}\right.
$$

with initial conditions $\mathbf{u}^{h, 0}=\mathbf{u}_{0}^{h}, \dot{\mathbf{u}}^{h, 0}=\dot{\mathbf{u}}_{0}^{h}, \ddot{\mathbf{u}}^{h, 0}=\ddot{\mathbf{u}}_{0}^{h}$. The following proposition holds:

Proposition 4. 1. If $\tilde{\beta}=0$, existence and uniqueness of (61) always holds since the scheme is fully explicit. 2. Let $\tilde{\beta}>0$. If

$$
(1+\theta)^{2} \leq C \gamma_{0}\left(1+\frac{\rho h^{2}}{\tau^{2} \tilde{\beta}}\right)
$$

where $C$ is a positive constant, then at each time-step n, Problem (61) admits one unique solution.

3. Suppose that $L^{n} \equiv 0$ for all $n \geq 0$. Then, for $\gamma_{0}$ sufficiently large, $\theta=1, \tilde{\gamma}=1$, and $\tilde{\beta}=1 / 2$, the following stability estimate holds for the solution to Problem (61) for all $n \geq 0$ :

$$
E_{1}^{h, n+1} \leq E_{1}^{h, n} .
$$

So the scheme (61) is unconditionally stable when $\theta=1, \tilde{\gamma}=1$ and $\tilde{\beta}=1 / 2$ (i.e., stable for all $h>0$ and all $\tau>0)$.

- A new time-marching scheme. We followed the ideas from [44] in the context of incompressible nonlinear elasticity and from [53] for penalized contact, where the authors design modified time-marching schemes to enforce energy conservation. The idea is to propose an hybrid discretization of the Nitsche-based contact term: the linear part of Problem (56) is treated with a conservative Crank-Nicolson scheme, whereas the non-linear part arising from contact is discretized with a linear combination of Crank-Nicolson and Midpoint schemes. This strategy is of interest since the resulting scheme is unconditionally stable in the symmetric case (for more properties, see $[26,27])$.

For $n \geq 0$, the fully discretized problem reads:

$$
\left\{\begin{array}{l}
\text { Find } \mathbf{u}^{h, n+1}, \dot{\mathbf{u}}^{h, n+1}, \ddot{\mathbf{u}}^{h, n+1} \in \mathbf{V}^{h} \text { such that: } \\
\mathbf{u}^{h, n+1}=\mathbf{u}^{h, n}+\tau \dot{\mathbf{u}}^{h, n+\frac{1}{2}} \\
\dot{\mathbf{u}}^{h, n+1}=\dot{\mathbf{u}}^{h, n}+\tau \ddot{\mathbf{u}}^{h, n+\frac{1}{2}}, \\
\left(\rho \ddot{\mathbf{u}}^{h, n+\frac{1}{2}}, \mathbf{v}^{h}\right)_{0, \Omega}+A_{\theta \gamma}^{\mathbf{n}}\left(\mathbf{u}^{h, n+\frac{1}{2}}, \mathbf{v}^{h}\right)+\int_{\Gamma_{C}} \frac{1}{\gamma} \Phi\left(\mathbf{u}^{h, n}, \mathbf{u}^{h, n+1}\right) \mathrm{P}_{\theta, \gamma}^{\mathbf{n}}\left(\mathbf{v}^{h}\right) d \Gamma=L^{n+\frac{1}{2}}\left(\mathbf{v}^{h}\right), \quad \forall \mathbf{v}^{h} \in \mathbf{V}^{h},
\end{array}\right.
$$

with the initial conditions $\mathbf{u}^{h, 0}=\mathbf{u}_{0}^{h}, \dot{\mathbf{u}}^{h, 0}=\dot{\mathbf{u}}_{0}^{h}, \ddot{\mathbf{u}}^{h, 0}=\ddot{\mathbf{u}}_{0}^{h}$ and with the following expression for $\Phi\left(\mathbf{u}^{h, n}, \mathbf{u}^{h, n+1}\right)$ :

$$
\Phi\left(\mathbf{u}^{h, n}, \mathbf{u}^{h, n+1}\right):=H\left(-\mathrm{P}_{1, \gamma}^{\mathbf{n}}\left(\mathbf{u}^{h, n}\right)\right)\left[\mathrm{P}_{1, \gamma}^{\mathbf{n}}\left(\mathbf{u}^{h, n+\frac{1}{2}}\right)\right]_{\mathbb{R}^{-}}+H\left(\mathrm{P}_{1, \gamma}^{\mathbf{n}}\left(\mathbf{u}^{h, n}\right)\right)\left[\mathrm{P}_{1, \gamma}^{\mathbf{n}}\left(\mathbf{u}^{h}\right)\right]_{\mathbb{R}^{-}}^{n+\frac{1}{2}} .
$$

Remark that $\left[\mathrm{P}_{1, \gamma}^{\mathbf{n}}\left(\mathbf{u}^{h}\right)\right]_{\mathbb{R}^{-}}^{n+\frac{1}{2}}=1 / 2\left(\left[\mathrm{P}_{1, \gamma}^{\mathbf{n}}\left(\mathbf{u}^{h, n}\right)\right]_{\mathbb{R}^{-}}+\left[\mathrm{P}_{1, \gamma}^{\mathbf{n}}\left(\mathbf{u}^{h, n+1}\right)\right]_{\mathbb{R}^{-}}\right)$represents the Crank-Nicolson part, whereas $\left[\mathrm{P}_{1, \gamma}^{\mathbf{n}}\left(\mathbf{u}^{h, n+\frac{1}{2}}\right)\right]_{\mathbb{R}^{-}}=\left[1 / 2\left(\mathrm{P}_{1, \gamma}^{\mathbf{n}}\left(\mathbf{u}^{h, n}\right)+\mathrm{P}_{1, \gamma}^{\mathbf{n}}\left(\mathbf{u}^{h, n+1}\right)\right)\right]_{\mathbb{R}^{-}}$stands for the Midpoint part. So, when $\mathrm{P}_{1, \gamma}^{\mathbf{n}}\left(\mathbf{u}^{h, n}\right)<0$, the Midpoint scheme is applied, and when $\mathrm{P}_{1, \gamma}^{\mathbf{n}}\left(\mathbf{u}^{h, n}\right)>0$, the Crank-Nicolson scheme is applied instead. When $\mathrm{P}_{1, \gamma}^{\mathbf{n}}\left(\mathbf{u}^{h, n}\right)=0$ both schemes coincide. 
The well-posedness and stability properties of the fully discrete scheme (62) are stated below.

Proposition 5. 1. If the condition below is satisfied

$$
(1+\theta)^{2} \leq C \gamma_{0}\left(1+\frac{\rho h^{2}}{\tau^{2}}\right)
$$

where $C$ is a positive constant, then at each time-step n, Problem (62) admits one unique solution.

2. Suppose that $L^{n} \equiv 0$ for all $n \geq 0$ and that Problem (62) is well-posed. Suppose also that $\theta=1$. The following stability estimate holds for the solution to Problem (62) for all $n \geq 0$ :

$$
E_{1}^{h, n+1} \leq E_{1}^{h, n} .
$$

So the scheme (62) is unconditionally stable when $\theta=1$ (i.e., stable for all $h>0$ and all $\tau>0$ ).

Remark 16. From Proposition 5 we observe that the scheme (62) is not exactly conservative. Nevertheless the energy dissipation caused by the scheme is almost negligible in practice, and reduces when the discretization parameters $h, \tau$ are taken smaller.

We next show an extension of the method to the Coulomb frictional case where $\mathscr{F} \geq 0$ still denotes the friction coefficient (the case $\mathscr{F}=0$ corresponds to the former frictionless case). Now the condition $\sigma_{\mathbf{t}}(\mathbf{u})=\mathbf{0}$ on $\Gamma_{C T}$ in (54) has to be changed with

$$
\begin{aligned}
& \dot{\mathbf{u}}_{\mathbf{t}}=\mathbf{0} \quad \Longrightarrow \quad\left|\sigma_{\mathbf{t}}(\mathbf{u})\right| \leq-\mathscr{F} \sigma_{n}(\mathbf{u}) \\
& \dot{\mathbf{u}}_{\mathbf{t}} \neq \mathbf{0} \Longrightarrow \quad \sigma_{\mathbf{t}}(\mathbf{u})=\mathscr{F} \sigma_{n}(\mathbf{u}) \frac{\dot{\mathbf{u}}_{\mathbf{t}}}{\left|\dot{\mathbf{u}}_{\mathbf{t}}\right|} .
\end{aligned}
$$

It is easy to check that the total mechanical energy $E(t)$ satisfies

$$
\frac{d}{d t} E(t) \leq L(t) \dot{\mathbf{u}}(t)
$$

In particular, when $L$ vanishes, we get energy dissipation contrary to the frictionless case: $E\left(t_{2}\right) \leq E\left(t_{1}\right)$, for all $0 \leq t_{1} \leq t_{2} \leq T$.

Next, the space semi-discretized Nitsche-based method for frictional unilateral contact problems in elastodynamics reads:

$$
\left\{\begin{array}{l}
\text { Find } \mathbf{u}^{h}:[0, T] \rightarrow \mathbf{V}^{h} \text { such that for } t \in[0, T]: \\
\left(\rho \ddot{\mathbf{u}}^{h}(t), \mathbf{v}^{h}\right)_{0, \Omega}+A_{\theta \gamma}\left(\mathbf{u}^{h}(t), \mathbf{v}^{h}\right)+\int_{\Gamma_{C}} \frac{1}{\gamma}\left[\mathrm{P}_{1, \gamma}^{\mathbf{n}}\left(\mathbf{u}^{h}(t)\right)\right]_{\mathbb{R}^{-}} \mathrm{P}_{\theta, \gamma}^{\mathbf{n}}\left(\mathbf{v}^{h}\right) d \Gamma \\
+\int_{\Gamma_{C}} \frac{1}{\gamma}\left[\sigma_{\mathbf{t}}\left(\mathbf{u}^{h}(t)\right)-\gamma \dot{\mathbf{u}}^{h}(t)\right]\left(-\mathscr{F}\left[\mathrm{P}_{1, \gamma}^{\mathbf{n}}\left(\mathbf{u}^{h}(t)\right)\right]_{\mathbb{R}^{-}}\right) \\
\mathbf{u}^{h}(0, \cdot)=\mathbf{u}_{\theta, \gamma}^{h}, \quad \dot{\mathbf{u}}^{h}(0, \cdot)=\dot{\mathbf{u}}_{0}^{h},
\end{array}\right.
$$

where $\mathbf{u}_{0}^{h}$ (resp. $\dot{\mathbf{u}}_{0}^{h}$ ) is a finite element approximation in $\mathbf{V}^{h}$ of the initial displacement $\mathbf{u}_{0}$ (resp. the initial velocity $\dot{\mathbf{u}}_{0}$ ). Using similar tools as in the frictionless case allows us to prove that for any $\mathscr{F} \geq 0, \theta \in \mathbb{R}, \gamma_{0}>0$, Problem (65) admits one unique solution $\mathbf{u}^{h} \in \mathscr{C}^{2}\left([0, T], \mathbf{V}^{h}\right)$. Further details and schemes can be found in [25].

We end this section with a numerical result that comes from [27] (see this reference for more details and other numerical tests). The corresponding test-case concerns multiple impacts of an elastic bar: in the absence of external volume forces, the bar is initially compressed. It is then released without initial velocity. It first impacts the rigid 
ground, located at $x=0$, then gets compressed once again and moves back to its initial position. This problem admits a closed-form solution which derivation and expression are detailed in [32]. Especially it has a periodic motion of period 3. At each period, the bar remains in contact with the rigid ground during one time unit (see Fig. 8). The chosen simulation time is $T=12$, so that we can observe 4 successive impacts.

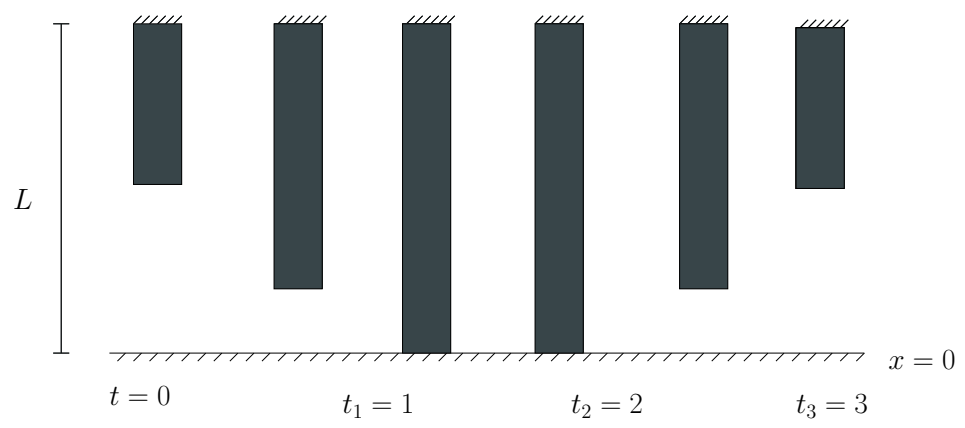

Fig. 8 Multiple impacts of an elastic bar. The bar is clamped at $x=L$ and the contact node is located at the bottom. The solution is periodic of period 3, with one impact during each period (here between $t=1$ and $t=2$ ).

We make comparisons to the modified mass method (see, e.g., [63]). The chosen method to compute the modified mass matrix is the simplest possible, since we set the entries associated with the contact node to 0 and no mass redistribution is considered (see also [34]). We combine the modified mass method either to the standard (mixed) method or to Nitsche's method for the treatment of contact conditions. The chosen time-marching scheme is CrankNicolson (Newmark with $\gamma=1 / 2, \beta=1 / 4$ ). These two methods are compared to the Nitsche-Hybrid scheme, with the same parameters and without modified mass. The results are depicted in Fig. 9.
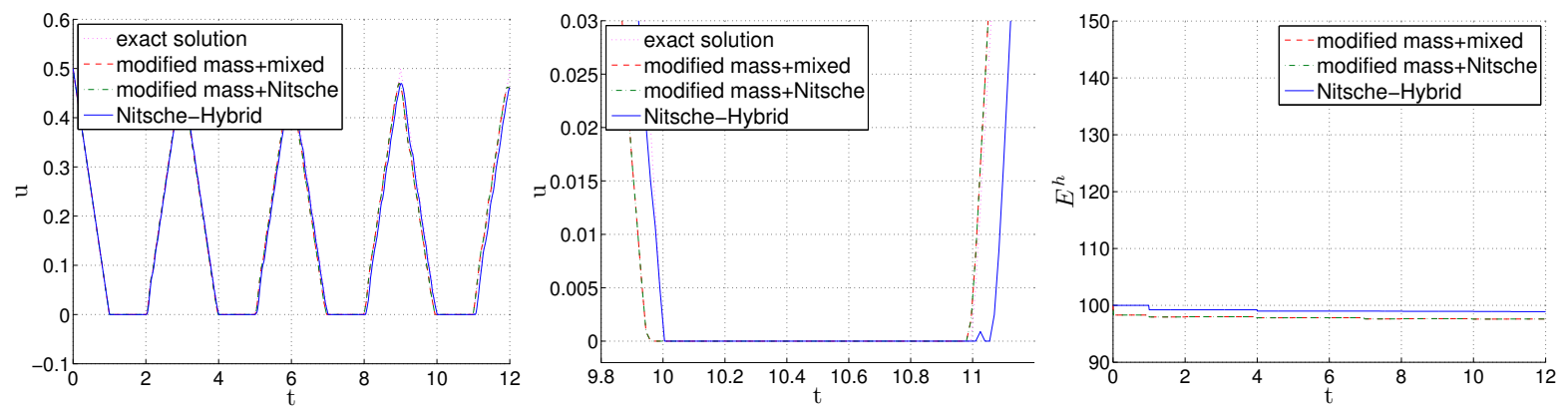

Fig. 9 Multiple impacts. Comparison of mixed with modified mass, Nitsche with modified mass and Nitsche-Hybrid methods. Displacement $u$ (left), zoom on displacement $u$ during the fourth impact (center) and discrete energy $E^{h}$ (right).

The three methods compare well and there is no significant difference: the displacement is free of spurious oscillations, the energy is quite well preserved, with only 1 or $2 \%$ of dissipation and some small spurious oscillations are still present on the contact pressure, that are of similar magnitude. This behaviour is well-known for modified mass combined to mixed discretization of the contact (see, e.g., [63, 34]). These results show that, also for Nitsche's discretization of the contact condition, the modified mass improves the quality of the solution in terms of spurious 
oscillations and energy conservation. In this test-case, the treatment through modified mass produces almost the same effects as the Hybrid time-marching scheme.

\section{Conclusion and perspectives}

Nitsche's method allows a numerical treatment of contact and friction in a simple manner since it remains a primal method, and is more robust than penalty, since it is consistent. For the Signorini problem, a rather complete numerical analysis can be carried out, to establish well-posedness and optimal convergence under assumptions on Nitsche's parameters similar to those commonly encountered for Dirichlet boundary conditions. The method can be extended to take into account various situations such as multi-body contact, large transformations and contact in elastodynamics. Most common friction's law such as Coulomb or Tresca can be formulated as well within this framework. Non-symmetric methods corresponding to $\theta=0$ and $\theta=-1$ reveal to be more robust numerically, though they imply a loss of symmetry in the tangent system.

Forthcoming studies may deal with numerical analysis in some situations in which results are lacking, such as contact in elastodynamics, or Coulomb's friction. The same method can be considered as well to discretize other categories of contact / friction problems, or other types of non-linear boundary conditions associated to variational inequalities.

Acknowledgements. The authors thank Erik Burman, the editorial board and Springer for the invitation to write this paper for their special volume. Moreover they thank the two anonymous referees for their constructive comments that helped to improve the paper. They thank also Thomas Boiveau and Susanne Claus for the GUFEM meeting and discussions. The first author thanks Région Bourgogne Franche-Comté for partial funding ("Convention Région 2015C-4991. Modèles mathématiques et méthodes numériques pour l'élasticité non-linéaire"), as well as Erik Burman and Miguel A. Fernández for some inspiring discussions on Nitsche's method.

\section{References}

1. Adams, R.A.: Sobolev spaces, Pure and Applied Mathematics, vol. 65. Academic Press, New York-London (1975)

2. Alart, P., Curnier, A.: A generalized Newton method for contact problems with friction. J Mec. Theor. Appl. 7(1), 67-82 (1988)

3. Annavarapu, C., Settgast, R.R., Johnson, S.M., Fu, P., Herbold, E.B.: A weighted Nitsche stabilized method for small-sliding contact on frictional surfaces. Comput. Methods Appl. Mech. Engrg. 283, 763-781 (2015). DOI 10.1016/j.cma.2014.09.030. URL http://dx.doi.org/10.1016/j.cma.2014.09.030

4. Armero, F., Petőcz, E.: Formulation and analysis of conserving algorithms for frictionless dynamic contact/impact problems. Comput. Methods Appl. Mech. Engrg. 158(3-4), 269-300 (1998). DOI 10.1016/S0045-7825(97)00256-9. URL http://dx. doi.org/10.1016/S0045-7825(97)00256-9

5. Arnold, D.: An interior penalty finite element method with discontinuous elements. SIAM J. Numer. Anal. 19, 742-760 (1982)

6. Ballard, P.: Steady sliding frictional contact problems in linear elasticity. J. Elasticity 110(1), 33-61 (2013). DOI 10.1007/ s10659-012-9381-6. URL http://dx.doi.org/10.1007/s10659-012-9381-6

7. Barbosa, H.J.C., Hughes, T.J.R.: Circumventing the Babuška-Brezzi condition in mixed finite element approximations of elliptic variational inequalities. Comput. Methods Appl. Mech. Engrg. 97(2), 193-210 (1992). DOI 10.1016/0045-7825(92)90163-E. URL http://dx.doi.org/10.1016/0045-7825(92)90163-E

8. Becker, R., Hansbo, P., Stenberg, R.: A finite element method for domain decomposition with non-matching grids. M2AN Math. Model. Numer. Anal. 37(2), 209-225 (2003)

9. Ben Belgacem, F.: Numerical simulation of some variational inequalities arisen from unilateral contact problems by the finite element methods. SIAM J. Numer. Anal. 37(4), 1198-1216 (2000). DOI 10.1137/S0036142998347966. URL http://dx. doi.org/10.1137/S0036142998347966 
10. Ben Belgacem, F., Renard, Y.: Hybrid finite element methods for the Signorini problem. Math. Comp. 72(243), 1117-1145 (2003). DOI 10.1090/S0025-5718-03-01490-X. URL http://dx. doi.org/10.1090/S0025-5718-03-01490-X

11. Boiveau, T., Burman, E.: A penalty-free Nitsche method for the weak imposition of boundary conditions in compressible and incompressible elasticity. IMA J. Numer. Anal. 36(2), 770-795 (2016). DOI 10.1093/imanum/drv042. URL http: / / dx . doi . org/10.1093/imanum/drv042

12. Brenner, S.C., Scott, L.R.: The mathematical theory of finite element methods, Texts in Applied Mathematics, vol. 15. SpringerVerlag, New York (2007)

13. Brezis, H.: Équations et inéquations non linéaires dans les espaces vectoriels en dualité. Ann. Inst. Fourier (Grenoble) 18(fasc. 1), 115-175 (1968)

14. Burman, E.: A penalty-free nonsymmetric Nitsche-type method for the weak imposition of boundary conditions. SIAM J. Numer. Anal. 50(4), 1959-1981 (2012). DOI 10.1137/10081784X. URL http://dx.doi.org/10.1137/10081784X

15. Burman, E., Hansbo, P.: Fictitious domain finite element methods using cut elements: II. A stabilized Nitsche's method. Comput. Methods Appl. Mech. Engrg. 2(4), 328-341 (2012)

16. Burman, E., Hansbo, P.: Deriving robust unfitted finite element methods from augmented lagrangian formulations. arXiv preprint arXiv: 1702.08340 (2017)

17. Burman, E., Hansbo, P., Larson, M.: Augmented Lagrangian finite element methods for contact problems. arXiv preprint arXiv:1609.03326 (2016)

18. Burman, E., Hansbo, P., Larson, M.G.: The Penalty Free Nitsche Method and Nonconforming Finite Elements for the Signorini Problem. arXiv preprint arXiv:1609.03745 (2016)

19. Burman, E., Hansbo, P., Larson, M.G., Stenberg, R.: Galerkin least squares finite element method for the obstacle problem. Comput. Meth. Appl. Mech. Engrg. 313, 362-374 (2017)

20. Chernov A. Maischak, M., Stephan, E.: A priori error estimates for hp penalty BEM for contact problems in elasticity. Comput. Methods Appl. Mech. Engrg. 196, 3871-3880 (2007)

21. Chouly, F.: An adaptation of Nitsche's method to the Tresca friction problem. J. Math. Anal. Appl. 411(1), 329-339 (2014). DOI 10.1016/j.jmaa.2013.09.019. URL http://dx.doi.org/10.1016/j.jmaa.2013.09.019

22. Chouly, F., Fabre, M., Hild, P., Pousin, J., Renard, Y.: Residual-based a posteriori error estimation for contact problems approximated by Nitsche's method. IMA J. Numer. Anal. To appear.

23. Chouly, F., Hild, P.: A Nitsche-based method for unilateral contact problems: numerical analysis. SIAM J. Numer. Anal. 51(2), 1295-1307 (2013). DOI 10.1137/12088344X. URL http: / / dx.doi.org/10.1137/12088344X

24. Chouly, F., Hild, P.: On convergence of the penalty method for unilateral contact problems. Appl. Numer. Math. 65(0), 2740 (2013). DOI 10.1016/j.apnum.2012.10.003. URL http://www.sciencedirect.com/science/article/pii/ S0168927412001924

25. Chouly, F., Hild, P., Renard, Y.: Nitsche method for dynamic contact with Coulomb friction. In preparation.

26. Chouly, F., Hild, P., Renard, Y.: A Nitsche finite element method for dynamic contact: 1. Space semi-discretization and timemarching schemes. ESAIM Math. Model. Numer. Anal. 49(2), 481-502 (2015). DOI 10.1051/m2an/2014041. URL http: //dx.doi.org/10.1051/m2an/2014041

27. Chouly, F., Hild, P., Renard, Y.: A Nitsche finite element method for dynamic contact: 2. Stability of the schemes and numerical experiments. ESAIM Math. Model. Numer. Anal. 49(2), 503-528 (2015). DOI 10.1051/m2an/2014046. URL http://dx. doi.org/10.1051/m2an/2014046

28. Chouly, F., Hild, P., Renard, Y.: Symmetric and non-symmetric variants of Nitsche's method for contact problems in elasticity: theory and numerical experiments. Math. Comp. 84(293), 1089-1112 (2015). DOI 10.1090/S0025-5718-2014-02913-X. URL http://dx.doi.org/10.1090/S0025-5718-2014-02913-X

29. Chouly, F., Mlika, R., Renard, Y.: An unbiased Nitsche's approximation of the frictional contact between two elastic structures (2015). URL https://hal archives-ouvertes.fr/hal-01240068. In revision.

30. Ciarlet, P.G.: The finite element method for elliptic problems, Handbook of Numerical Analysis (eds. P.G. Ciarlet and J.L. Lions), vol. II. North-Holland Publishing Co., Amsterdam (1991)

31. Dabaghi, F., Petrov, A., Pousin, J., Renard, Y.: Convergence of mass redistribution method for the one-dimensional wave equation with a unilateral constraint at the boundary. M2AN Math. Model. Numer. Anal. 48, 1147-1169 (2014). URL http: //www . esaim-m2an.org/article_S0764583X13001337

32. Dabaghi, F., Petrov, A., Pousin, J., Renard, Y.: A robust finite element redistribution approach for elastodynamic contact problems. Appl. Numer. Math. 103, 48-71 (2016). DOI 10.1016/j.apnum.2015.12.004. URL http://dx.doi.org/10.1016/j . apnum.2015.12.004

33. Di Pietro, D.A., Ern, A.: Mathematical aspects of discontinuous Galerkin methods, Mathématiques \& Applications (Berlin) [Mathematics \& Applications], vol. 69. Springer, Heidelberg (2012). DOI 10.1007/978-3-642-22980-0. URL http: //dx.doi.org/10.1007/978-3-642-22980-0

34. Doyen, D., Ern, A., Piperno, S.: Time-integration schemes for the finite element dynamic Signorini problem. SIAM J. Sci. Comput. 33(1), 223-249 (2011). DOI 10.1137/100791440. URL http://dx.doi.org/10 .1137/100791440 
35. Drouet, G., Hild, P.: Optimal convergence for discrete variational inequalities modelling Signorini contact in 2D and 3D without additional assumptions on the unknown contact set. SIAM J. Numer. Anal. 53(3), 1488-1507 (2015). DOI 10.1137/140980697. URL http://dx.doi.org/10.1137/140980697

36. Duvaut, G., Lions, J.L.: Les inéquations en mécanique et en physique, Travaux et Recherches Mathématiques, vol. 21. Dunod, Paris (1972)

37. Eck, C., Jarušek, J., Krbec, M.: Unilateral contact problems, Pure and Applied Mathematics (Boca Raton), vol. 270. Chapman \& Hall/CRC, Boca Raton, FL (2005). DOI 10.1201/9781420027365. URL http://dx.doi.org/10.1201/ 9781420027365

38. Ern, A., Guermond, J.L.: Theory and practice of finite elements, Applied Mathematical Sciences, vol. 159. Springer-Verlag, New York (2004)

39. Fabre, M., Pousin, J., Renard, Y.: A fictitious domain method for frictionless contact problems in elasticity using Nitsche's method. SMAI J. Comput. Math. 2, 19-50 (2016)

40. Fichera, G.: Problemi elastostatici con vincoli unilaterali: Il problema di Signorini con ambigue condizioni al contorno. Atti Accad. Naz. Lincei Mem. Cl. Sci. Fis. Mat. Natur. Sez. I (8) 7, 91-140 (1963/1964)

41. Galántai, A.: Properties and construction of NCP functions. Comput. Optim. Appl. 52(3), 805-824 (2012). DOI 10.1007/ s10589-011-9428-9. URL http://dx.doi.org/10.1007/s10589-011-9428-9

42. Glowinski, R.: Numerical methods for nonlinear variational problems. Springer Series in Computational Physics. Springer-Verlag, New York (1984). DOI 10.1007/978-3-662-12613-4. URL http://dx.doi.org/10.1007/978-3-662-12613-4

43. Glowinski, R., Le Tallec, P.: Augmented Lagrangian and operator-splitting methods in nonlinear mechanics, SIAM Studies in Applied Mathematics, vol. 9. Society for Industrial and Applied Mathematics (SIAM), Philadelphia, PA (1989)

44. Gonzalez, O.: Exact energy and momentum conserving algorithms for general models in nonlinear elasticity. Comput. Methods Appl. Mech. Engrg. 190(13-14), 1763-1783 (2000). DOI 10.1016/S0045-7825(00)00189-4. URL http://dx.doi.org/ $10.1016 / \mathrm{S} 0045-7825(00) 00189-4$

45. Han, W., Sofonea, M.: Quasistatic contact problems in viscoelasticity and viscoplasticity, AMS/IP Studies in Advanced Mathematics, vol. 30. American Mathematical Society, Providence, RI (2002)

46. Hansbo, A., Hansbo, P.: A finite element method for the simulation of strong and weak discontinuities in solid mechanics. Comput. Methods Appl. Mech. Engrg. 193(33-35), 3523-3540 (2004). DOI 10.1016/j.cma.2003.12.041. URL http://dx.doi.org/ $10.1016 / j . \mathrm{cma} .2003 .12 .041$

47. Hansbo, P.: Nitsche's method for interface problems in computational mechanics. GAMM-Mitt. 28(2), 183-206 (2005)

48. Hansbo, P., Rashid, A., Salomonsson, K.: Least-squares stabilized augmented Lagrangian multiplier method for elastic contact. Finite Elem. Anal. Des. 116, 32-37 (2016). DOI 10.1016/j.finel.2016.03.005. URL http://dx.doi.org/10.1016/j. finel.2016.03.005

49. Haslinger, J.: Finite element analysis for unilateral problems with obstacles on the boundary. Apl. Mat. 22(3), 180-188 (1977)

50. Haslinger, J., Hlaváček, I.: Approximation of the Signorini problem with friction by a mixed finite element method. J. Math. Anal. Appl. 86(1), 99-122 (1982). DOI 10.1016/0022-247X(82)90257-8. URL http://dx.doi.org/10.1016/ $0022-247 X(82) 90257-8$

51. Haslinger, J., Hlaváček, I., Nečas, J.: Numerical methods for unilateral problems in solid mechanics, Handbook of Numerical Analysis (eds. P.G. Ciarlet and J.L. Lions), vol. IV. North-Holland Publishing Co., Amsterdam (1996)

52. Haslinger, J., Renard, Y.: A new fictitious domain approach inspired by the extended finite element method. SIAM J. Numer. Anal. 47(2), 1474-1499 (2009). DOI 10.1137/070704435. URL http://dx.doi.org/10.1137/070704435

53. Hauret, P., Le Tallec, P.: Energy-controlling time integration methods for nonlinear elastodynamics and low-velocity impact. Comput. Methods Appl. Mech. Engrg. 195(37-40), 4890-4916 (2006). DOI 10.1016/j.cma.2005.11.005. URL http://dx. doi.org/10.1016/j.cma.2005.11.005

54. Heintz, P., Hansbo, P.: Stabilized Lagrange multiplier methods for bilateral elastic contact with friction. Comput. Methods Appl. Mech. Engrg. 195(33-36), 4323-4333 (2006). DOI 10.1016/j.cma.2005.09.008. URL http://dx.doi.org/10.1016/j. $\mathrm{cma} .2005 .09 .008$

55. Hild, P.: Numerical implementation of two nonconforming finite element methods for unilateral contact. Comput. Methods Appl. Mech. Engrg. 184(1), 99-123 (2000). DOI 10.1016/S0045-7825(99)00096-1. URL http: //dx. doi .org/10.1016/ S0045-7825 (99) 00096-1

56. Hild, P., Lleras, V.: Residual error estimators for Coulomb friction. SIAM J. Numer. Anal. 47(5), 3550-3583 (2009). DOI 10.1137/070711554. URL http://dx.doi.org/10.1137/070711554

57. Hild, P., Nicaise, S.: Residual a posteriori error estimators for contact problems in elasticity. M2AN Math. Model. Numer. Anal. 41(5), 897-923 (2007). DOI 10.1051/m2an:2007045. URL http://dx.doi.org/10.1051/m2an:2007045

58. Hild, P., Renard, Y.: A stabilized Lagrange multiplier method for the finite element approximation of contact problems in elastostatics. Numer. Math. 115(1), 101-129 (2010). DOI 10.1007/s00211-009-0273-z. URL http://dx.doi.org/10.1007/ s00211-009-0273-z 
59. Hild, P., Renard, Y.: An improved a priori error analysis for finite element approximations of Signorini's problem. SIAM J. Numer. Anal. 50(5), 2400-2419 (2012). DOI 10.1137/110857593. URL http://dx.doi.org/10.1137/110857593

60. Hüeber, S., Wohlmuth, B.I.: An optimal a priori error estimate for nonlinear multibody contact problems. SIAM J. Numer. Anal. 43(1), 156-173 (2005). DOI 10.1137/S0036142903436678. URL http://dx.doi.org/10.1137/ S0036142903436678

61. Juntunen, M.: On the connection between the stabilized Lagrange multiplier and Nitsche's methods. Numer. Math. 131(3), 453471 (2015). DOI 10.1007/s00211-015-0701-1. URL http://dx.doi.org/10.1007/s00211-015-0701-1

62. Juntunen, M., Stenberg, R.: Nitsche's method for general boundary conditions. Math. Comp. 78(267), 1353-1374 (2009). DOI 10.1090/S0025-5718-08-02183-2. URL http://dx.doi.org/10.1090/S0025-5718-08-02183-2

63. Khenous, H.B., Laborde, P., Renard, Y.: Mass redistribution method for finite element contact problems in elastodynamics. Eur. J. Mech. A Solids 27(5), 918-932 (2008). DOI 10.1016/j.euromechsol.2008.01.001. URL http: //dx. doi .org/10.1016/ j.euromechsol.2008.01.001

64. Kikuchi, N., Oden, J.T.: Contact problems in elasticity: a study of variational inequalities and finite element methods, SIAM Studies in Applied Mathematics, vol. 8. Society for Industrial and Applied Mathematics (SIAM), Philadelphia, PA (1988)

65. Kikuchi, N., Song, Y.J.: Penalty-finite-element approximation of a class of unilateral problems in linear elasticity. Quart. Appl. Math. 39, 1-22 (1981)

66. Kim, J.U.: A boundary thin obstacle problem for a wave equation. Comm. Partial Differential Equations 14(8-9), 1011-1026 (1989). DOI 10.1080/03605308908820640. URL http://dx.doi.org/10.1080/03605308908820640

67. Laborde, P., Renard, Y.: Fixed point strategies for elastostatic frictional contact problems. Math. Methods Appl. Sci. 31(4), 415441 (2008). DOI 10.1002/mma.921. URL http://dx.doi.org/10.1002/mma. 921

68. Laursen, T.A.: Computational contact and impact mechanics. Springer-Verlag, Berlin (2002)

69. Laursen, T.A., Chawla, V.: Design of energy conserving algorithms for frictionless dynamic contact problems. Internat. J. Numer. Methods Engrg. 40(5), 863-886 (1997). DOI 10.1002/(SICI)1097-0207(19970315)40:5<863::AID-NME92 >3.0.CO;2-V. URL http://dx.doi.org/10.1002/(SICI) 1097-0207 (19970315) $40: 5<863:$ : AID-NME92>3.0.CO;2-V

70. Lebeau, G., Schatzman, M.: A wave problem in a half-space with a unilateral constraint at the boundary. J. Differential Equations 53(3), 309-361 (1984). DOI 10.1016/0022-0396(84)90030-5. URL http://dx. doi.org/10.1016/0022-0396 (84) $90030-5$

71. Lions, J.L.: Quelques méthodes de résolution des problèmes aux limites non linéaires. Dunod, Paris (1969)

72. Lleras, V.: Modélisation, analyse et simulation de problèmes de contact en mécanique des solides et des fluides. Ph.D. thesis, Besançon (2009)

73. Lüthen, N., Juntunen, M., Stenberg, R.: An improved a priori error analysis of Nitsche's method for Robin boundary conditions. arXiv preprint arXiv:1502.06515 (2015)

74. Mlika, R., Renard, Y., Chouly, F.: An unbiased Nitsche's formulation of large deformation frictional contact and self-contact (2017). URL https://hal.archives-ouvertes.fr/hal-01427872. In revision.

75. Nitsche, J.: Über ein Variationsprinzip zur Lösung von Dirichlet-Problemen bei Verwendung von Teilräumen, die keinen Randbedingungen unterworfen sind. Abhandlungen aus dem Mathematischen Seminar der Universität Hamburg 36, 9-15 (1971)

76. Oden, J.T.: Penalty-finite-element methods for constrained problems in elasticity. Symposium on finite element methods pp. 122 (1981). URL https://www. ices.utexas.edu/sites/oden/wp-content/uploads/2013/06/1981-008. penalty-finite_element.pdf

77. Oden, J.T., Kikuchi, N.: Finite element methods for constrained problems in elasticity. Internat. J. Numer. Meth. Engrg. 18, 701-725 (1982)

78. Oden, J.T., Kim, S.J.: Interior penalty methods for finite element approximations of the Signorini problem in elastostatics. Comput. Math. Appl. 8(1), 35-56 (1982). DOI 10.1016/0898-1221(82)90038-4. URL http://dx.doi.org/10.1016/ $0898-1221(82) 90038-4$

79. Pipping, E., Sander, O., Kornhuber, R.: Variational formulation of rate- and state-dependent friction problems. ZAMM Z. Angew. Math. Mech. 95(4), 377-395 (2015). DOI 10.1002/zamm.201300062. URL http://dx.doi.org/10.1002/ zamm. 201300062

80. Poulios, K., Renard, Y.: An unconstrained integral approximation of large sliding frictional contact between deformable solids. Comput. Struct. (153), 75-90 (2015)

81. Renard, Y.: Generalized Newton's methods for the approximation and resolution of frictional contact problems in elasticity. Comput. Methods Appl. Mech. Engrg. 256, 38-55 (2012)

82. Renard, Y.: Approximation des conditions de contact en élastostatique : méthode de Nitsche et lien avec le lagrangien augmenté proximal (2015). Proceedings of the Besançon Week on Numerical Analysis.

83. Sauer, R.A., DeLorenzis, L.: An unbiased computational contact formulation for 3D friction. Internat. J. Numer. Methods Engrg. 101(4), 251-280 (2015). DOI 10.1002/nme.4794. URL http: / / dx. doi .org/10 .1002/nme. 4794

84. Scarpini, F., Vivaldi, M.A.: Error estimates for the approximation of some unilateral problems. RAIRO Anal. Numér. 11(2), 197-208 (1977) 
85. Stenberg, R.: On some techniques for approximating boundary conditions in the finite element method. J. Comput. Appl. Math. 63(1-3), 139-148 (1995). DOI 10.1016/0377-0427(95)00057-7. URL http://dx. doi .org/10.1016/0377-0427 (95) $00057-7$

86. Thomée, V.: Galerkin finite element methods for parabolic problems, Springer Series in Computational Mathematics, vol. 25. Springer-Verlag, Berlin (1997)

87. Verfürth, R.: A review of a posteriori error estimation techniques for elasticity problems. Comput. Methods Appl. Mech. Engrg. 176(1-4), 419-440 (1999). DOI 10.1016/S0045-7825(98)00347-8. URL http://dx.doi.org/10.1016/ S $0045-7825$ (98) $00347-8$

88. Wohlmuth, B.I.: A residual based error estimator for mortar finite element discretizations. Numer. Math. 84(1), 143-171 (1999). DOI 10.1007/s002110050467. URL http://dx.doi.org/10.1007/s002110050467

89. Wohlmuth, B.I.: Variationally consistent discretization schemes and numerical algorithms for contact problems. Acta Numer. 20, 569-734 (2011). DOI 10.1017/S0962492911000079. URL http: / / dx.doi.org/10 .1017/S0962492911000079

90. Wriggers, P.: Computational Contact Mechanics. Wiley (2002)

91. Wriggers, P., Zavarise, G.: A formulation for frictionless contact problems using a weak form introduced by Nitsche. Comput. Mech. 41, 407-420 (2008). DOI 10.1007/s00466-007-0196-4. URL http://dx.doi.org/10.1007/ s00466-007-0196-4 